\title{
Bottlenecks to interstellar sulfur chemistry
}

\section{Sulfur-bearing hydrides in UV-illuminated gas and grains}

\author{
J. R. Goicoechea ${ }^{1}$, A. Aguado ${ }^{2}$, S. Cuadrado ${ }^{1}$, O. Roncero ${ }^{1}$, J. Pety ${ }^{3}$, E. Bron ${ }^{4}$, \\ A. Fuente ${ }^{5}$, D. Riquelme ${ }^{6}$, E. Chapillon ${ }^{3,7}$, C. Herrera ${ }^{3}$, and C. A. Duran ${ }^{6,8}$
}

\begin{abstract}
1 Instituto de Física Fundamental (CSIC). Calle Serrano 121-123, 28006, Madrid, Spain. e-mail: javier.r.goicoechea@csic.es 2 Facultad de Ciencias. Universidad Autónoma de Madrid, 28049 Madrid, Spain.

3 Institut de Radioastronomie Millimétrique (IRAM), Grenoble, France.

${ }^{4}$ LERMA, Observatoire de Paris, PSL Research University, CNRS, Sorbonne Universités, 92190 Meudon, France.

5 Observatorio Astronómico Nacional (OAN), Alfonso XII, 3, 28014 Madrid, Spain.

${ }^{6}$ Max-Planck-Institut für Radioastronomie, Auf dem Hügel 69, 53121 Bonn, Germany.

7 OASU/LAB-UMR5804, CNRS, Université Bordeaux, 33615 Pessac, France.

8 European Southern Observatory, Alonso de Cordova 3107, Vitacura, Santiago, Chile.
\end{abstract}

Received 25 October 2020 / Accepted 23 December 2020

\begin{abstract}
Hydride molecules lie at the base of interstellar chemistry, but the synthesis of sulfuretted hydrides is poorly understood and their abundances often crudely constrained. Motivated by new observations of the Orion Bar photodissociation region (PDR) - $1^{\prime \prime}$ resolution ALMA images of $\mathrm{SH}^{+}$; IRAM 30m detections of bright $\mathrm{H}_{2}^{32} \mathrm{~S}, \mathrm{H}_{2}^{34} \mathrm{~S}$, and $\mathrm{H}_{2}^{33} \mathrm{~S}$ lines; $\mathrm{H}_{3} \mathrm{~S}^{+}$(upper limits); and SOFIA/GREAT observations of SH (upper limits) - we perform a systematic study of the chemistry of sulfur-bearing hydrides. We self-consistently determine their column densities using coupled excitation, radiative transfer as well as chemical formation and destruction models. We revise some of the key gas-phase reactions that lead to their chemical synthesis. This includes ab initio quantum calculations of the vibrational-state-dependent reactions $\mathrm{SH}^{+}+\mathrm{H}_{2}(v) \rightleftarrows \mathrm{H}_{2} \mathrm{~S}^{+}+\mathrm{H}$ and $\mathrm{S}+\mathrm{H}_{2}(v) \rightleftarrows \mathrm{SH}+\mathrm{H}$. We find that reactions of UV-pumped $\mathrm{H}_{2}(v \geq 2)$ molecules with $\mathrm{S}^{+}$ions explain the presence of $\mathrm{SH}^{+}$in a high thermal-pressure gas component, $P_{\text {th }} / k \approx 10^{8} \mathrm{~cm}^{-3} \mathrm{~K}, \mathrm{close}$ to the $\mathrm{H}_{2}$ dissociation front (at $A_{V}<2 \mathrm{mag}$ ). These PDR layers are characterized by no or very little depletion of elemental sulfur from the gas. However, subsequent hydrogen abstraction reactions of $\mathrm{SH}^{+}, \mathrm{H}_{2} \mathrm{~S}^{+}$, and $\mathrm{S}$ atoms with vibrationally excited $\mathrm{H}_{2}$, fail to form enough $\mathrm{H}_{2} \mathrm{~S}^{+}, \mathrm{H}_{3} \mathrm{~S}^{+}$, and $\mathrm{SH}$ to ultimately explain the observed $\mathrm{H}_{2} \mathrm{~S}$ column density $\left(\sim 2.5 \times 10^{14} \mathrm{~cm}^{-2}\right.$, with an ortho-to-para ratio of $2.9 \pm 0.3$; consistent with the high-temperature statistical value). To overcome these bottlenecks, we build PDR models that include a simple network of grain surface reactions leading to the formation of solid $\mathrm{H}_{2} \mathrm{~S}\left(\mathrm{~s}-\mathrm{H}_{2} \mathrm{~S}\right)$. The higher adsorption binding energies of $\mathrm{S}$ and $\mathrm{SH}$ suggested by recent studies imply that $\mathrm{S}$ atoms adsorb on grains (and form s- $\mathrm{H}_{2} \mathrm{~S}$ ) at warmer dust temperatures $\left(T_{d}<50 \mathrm{~K}\right.$ ) and closer to the UV-illuminated edges of molecular clouds. We show that everywhere s- $\mathrm{H}_{2} \mathrm{~S}$ mantles form(ed), gas-phase $\mathrm{H}_{2} \mathrm{~S}$ emission lines will be detectable. Photodesorption and, to a lesser extent, chemical desorption, produce roughly the same $\mathrm{H}_{2} \mathrm{~S}$ column density (a few $10^{14} \mathrm{~cm}^{-2}$ ) and abundance peak (a few $10^{-8}$ ) nearly independently of $n_{\mathrm{H}}$ and $G_{0}$. This agrees with the observed $\mathrm{H}_{2} \mathrm{~S}$ column density in the Orion Bar as well as at the edges of dark clouds without invoking substantial depletion of elemental sulfur abundances.
\end{abstract}

Key words. Astrochemistry — line: identification — ISM: clouds — (ISM:) photon-dominated region (PDR) — ISM: clouds

\section{Introduction}

Hydride molecules play a pivotal role in interstellar chemistry (e.g., Gerin et al. 2016), being among the first molecules to form in diffuse interstellar clouds and at the UV-illuminated edges of dense star-forming clouds, so-called photodissociation regions (PDRs; Hollenbach \& Tielens 1997). Sulfur is on the top ten list of most abundant cosmic elements and it is particularly relevant for astrochemistry and star-formation studies. Its low ionization potential $(10.4 \mathrm{eV})$ makes the photoionization of $\mathrm{S}$ atoms a dominant source of electrons in molecular gas at intermediate visual extinctions $A_{V} \simeq 2$ - 4 mag (Sternberg \& Dalgarno 1995; Goicoechea et al. 2009; Fuente et al. 2016).

The sulfur abundance, $[\mathrm{S} / \mathrm{H}]$, in diffuse clouds (e.g., Howk et al. 2006) is very close to the $[\mathrm{S} / \mathrm{H}]$ measured in the solar photosphere $\left([\mathrm{S} / \mathrm{H}]_{\odot} \simeq 1.4 \times 10^{-5}\right.$; Asplund et al. 2009). Still, the observed abundances of S-bearing molecules in diffuse and translucent molecular clouds $\left(n_{\mathrm{H}} \simeq 10^{2}-10^{3} \mathrm{~cm}^{-3}\right)$ make up a very small fraction, $<1 \%$, of the sulfur nuclei (mostly locked as $\mathrm{S}^{+}$; Tieftrunk et al. 1994; Turner 1996; Lucas \& Liszt 2002; Neufeld et al. 2015). In colder dark clouds and dense cores shielded from stellar UV radiation, most sulfur is expected in molecular form. However, the result of adding the abundances of all detected gas-phase S-bearing molecules is typically a factor of $\sim 10^{2}-10^{3}$ lower than $[\mathrm{S} / \mathrm{H}]_{\odot}$ (e.g., Fuente et al. 2019). Hence, it is historically assumed that sulfur species deplete on grain mantles at cold temperatures and high densities (e.g., Graedel et al. 1982; Millar \& Herbst 1990; Agúndez \& Wakelam 2013). However, recent chemical models predict that the major sulfur reservoir in dark clouds can be either gas-phase neutral S atoms (Vidal et al. 2017; Navarro-Almaida et al. 2020) or organo-sulfur species trapped on grains (Laas \& Caselli 2019). Unfortunately, it is difficult to overcome this dichotomy from an observational perspective. In particular, no ice carrier of an abundant sulfur reservoir other than solid OCS (hereafter s-OCS, with an abundance of $\sim 10^{-8}$ with respect to $\mathrm{H}$ nuclei; Palumbo et al. 
1997) has been convincingly identified. Considering the large abundances of water ice $\left(\mathrm{s}-\mathrm{H}_{2} \mathrm{O}\right)$ grain mantles in dense molecular clouds and cold protostellar envelopes (see reviews by van Dishoeck 2004; Gibb et al. 2004; Dartois 2005), one may also expect hydrogen sulfide $\left(\mathrm{s}-\mathrm{H}_{2} \mathrm{~S}\right)$ to be the dominant sulfur reservoir. Indeed, $\mathrm{s}-\mathrm{H}_{2} \mathrm{~S}$ is the most abundant $\mathrm{S}$-bearing ice in comets such as $67 \mathrm{P} /$ Churyumov-Gerasimenko (Calmonte et al. 2016). However, only upper limits to the $\mathrm{s}-\mathrm{H}_{2} \mathrm{~S}$ abundance of $\lesssim 1 \%$ relative to water ice have so far been estimated toward a few interstellar sightlines (e.g., Smith 1991; Jiménez-Escobar \& Muñoz Caro 2011). These values imply a maximum $\mathrm{s}-\mathrm{H}_{2} \mathrm{~S}$ ice abundance of several $10^{-6}$ with respect to $\mathrm{H}$ nuclei. Still, this upper limit could be higher if $\mathrm{s}-\mathrm{H}_{2} \mathrm{~S}$ ices are well mixed with $\mathrm{s}-\mathrm{H}_{2} \mathrm{O}$ and s-CO ices (Brittain et al. 2020).

The bright rims of molecular clouds illuminated by nearby massive stars are intermediate environments between diffuse and cold dark clouds. Such environments host the transition from ionized $\mathrm{S}^{+}$to neutral atomic $\mathrm{S}$, as well as the gradual formation of S-bearing molecules (Sternberg \& Dalgarno 1995). In one prototypical low-illumination PDR, the edge of the Horsehead nebula, Goicoechea et al. (2006) inferred very modest gas-phase sulfur depletions. In addition, the detection of narrow sulfur radio recombination lines in dark clouds (implying the presence of $\mathrm{S}^{+}$; Pankonin \& Walmsley 1978) is an argument against large sulfur depletions in the mildly illuminated surfaces of these clouds. The presence of new S-bearing molecules such as $\mathrm{S}_{2} \mathrm{H}$, the first (and so far only) doubly sulfuretted species detected in a PDR (Fuente et al. 2017), suggests that the chemical pathways leading to the synthesis of sulfuretted species are not well constrained; and that the list of S-bearing molecules is likely not complete.

Interstellar sulfur chemistry is unusual compared to that of other elements in that none of the simplest species, $\mathrm{X}=\mathrm{S}, \mathrm{S}^{+}, \mathrm{SH}, \mathrm{SH}^{+}$, or $\mathrm{H}_{2} \mathrm{~S}^{+}$, react exothermically with $\mathrm{H}_{2}(v=0)$ in the initiation reactions $\mathrm{X}+\mathrm{H}_{2} \rightarrow \mathrm{XH}+\mathrm{H}$ (so-called hydrogen abstraction reactions). Hence, one would expect a slow sulfur chemistry and very low abundances of $\mathrm{SH}^{+}$(sulfanylium) and $\mathrm{SH}$ (mercapto) radicals in cold interstellar gas. However, $\mathrm{H}_{2} \mathrm{~S}$ (Lucas \& Liszt 2002), $\mathrm{SH}^{+}$(Menten et al. 2011; Godard et al. 2012), and SH (Neufeld et al. 2012, 2015) have been detected in low-density diffuse clouds $\left(n_{\mathrm{H}} \lesssim 100 \mathrm{~cm}^{-3}\right)$ through absorption measurements of their ground-state rotational lines ${ }^{1}$. In UV-illuminated gas, most sulfur atoms are ionized, but the very high endothermicity of reaction

$\mathrm{S}^{+}\left({ }^{4} S\right)+\mathrm{H}_{2}\left({ }^{1} \Sigma^{+}, v=0\right) \rightleftarrows \mathrm{SH}^{+}\left({ }^{3} \Sigma^{-}\right)+\mathrm{H}\left({ }^{2} S\right)$

( $E / k=9860 \mathrm{~K}$, e.g., Zanchet et al. 2013a, 2019) prevents this reaction from being efficient unless the gas is heated to very high temperatures. In diffuse molecular clouds (on average at $T_{\mathrm{k}} \sim 100 \mathrm{~K}$ ), the formation of $\mathrm{SH}^{+}$and $\mathrm{SH}$ only seems possible in the context of local regions of overheated gas subjected to magnetized shocks (Pineau des Forets et al. 1986) or in dissipative vortices of the interstellar turbulent cascade (Godard et al. 2012, 2014). In these tiny pockets $(\sim 100 \mathrm{AU}$ in size $)$, the gas would attain the hot temperatures $\left(T_{\mathrm{k}} \simeq 1000 \mathrm{~K}\right)$ and/or ionneutral drift needed to overcome the endothermicities of the above hydrogen abstraction reactions (see, e.g., Neufeld et al. 2015).

Dense PDRs $\left(n_{\mathrm{H}} \simeq 10^{3}-10^{6} \mathrm{~cm}^{-3}\right)$ offer a complementary environment to study the first steps of sulfur chemistry. Because of their higher densities and more quiescent gas, fast shocks or turbulence dissipation do not contribute to the gas heating. Instead, the molecular gas is heated to $T_{\mathrm{k}} \lesssim 500 \mathrm{~K}$ by mechanisms

\footnotetext{
1 SH was first reported by IR spectroscopy toward the cirumstellar envelope around the evolved star R Andromedae (Yamamura et al. 2000).
}

that depend on the flux of far-UV photons (FUV; $E<13.6 \mathrm{eV}$ ). A different perspective of the $\mathrm{H}_{2}(v)$ reactivity emerges because certain endoergic reactions become exoergic and fast when a significant fraction of the $\mathrm{H}_{2}$ reagents are radiatively pumped to vibrationally excited states $v \geq 1$ (Stecher \& Williams 1972; Freeman \& Williams 1982; Tielens \& Hollenbach 1985; Sternberg \& Dalgarno 1995). In this case, state-specific reaction rates for $\mathrm{H}_{2}(v, J)$ are needed to make realistic predictions of the abundance of the product XH (Agúndez et al. 2010; Zanchet et al. 2013b; Faure et al. 2017). The presence of abundant FUV-pumped $\mathrm{H}_{2}(v \geq 1)$ triggers a nonthermal "hot" chemistry. Indeed, $\mathrm{CH}^{+}$ and $\mathrm{SH}^{+}$emission lines have been detected in the Orion Bar PDR (Nagy et al. 2013; Goicoechea et al. 2017) where $\mathrm{H}_{2}$ lines up to $v=10$ have been detected as well (Kaplan et al. 2017).

In this study we present a systematic (observational and modeling) study of the chemistry of S-bearing hydrides in FUV-illuminated gas. We try to answer the question of whether gas-phase reactions of $\mathrm{S}$ atoms and $\mathrm{SH}^{+}$molecules with vibrationally excited $\mathrm{H}_{2}$ can ultimately explain the presence of abundant $\mathrm{H}_{2} \mathrm{~S}$, or if grain surface chemistry has to be invoked.

The paper is organized as follows. In Sects. 2 and 3 we report on new observations of $\mathrm{H}_{2}^{32} \mathrm{~S}, \mathrm{H}_{2}^{34} \mathrm{~S}, \mathrm{H}_{2}^{33} \mathrm{~S}, \mathrm{SH}^{+}, \mathrm{SH}$, and $\mathrm{H}_{3} \mathrm{~S}^{+}$ emission lines toward the Orion Bar. In Sect. 4 we study their excitation and derive their column densities. In Sect. 6 we discuss their abundances in the context of updated PDR models, with emphasis on the role of hydrogen abstraction reactions

$\mathrm{SH}^{+}\left({ }^{3} \Sigma^{-}\right)+\mathrm{H}_{2}\left({ }^{1} \Sigma^{+}\right) \rightleftarrows \mathrm{H}_{2} \mathrm{~S}^{+}\left({ }^{2} \mathrm{~A}^{\prime}\right)+\mathrm{H}\left({ }^{2} S\right)$,

$\mathrm{H}_{2} \mathrm{~S}^{+}\left({ }^{2} A^{\prime}\right)+\mathrm{H}_{2}\left({ }^{1} \Sigma^{+}\right) \rightleftarrows \mathrm{H}_{3} \mathrm{~S}^{+}\left(\mathrm{X}^{1} A_{1}\right)+\mathrm{H}\left({ }^{2} S\right)$,

$\mathrm{S}\left({ }^{3} P\right)+\mathrm{H}_{2}\left({ }^{1} \Sigma^{+}\right) \rightleftarrows \mathrm{SH}\left(\mathrm{X}^{2} \Pi\right)+\mathrm{H}\left({ }^{2} S\right)$,

photoreactions, and grain surface chemistry. In Sect. 5 we summarize the $a b$ initio quantum calculations we carried out to determine the state-dependent rates of reactions (2) and (4). Details of these calculations are given in Appendices A and B.

\section{Observations of S-bearing hydrides}

\subsection{The Orion Bar}

At an adopted distance of $\sim 414 \mathrm{pc}$, the Orion Bar is an interface of the Orion molecular cloud and the Huygens $\mathrm{H}_{\text {II }}$ region that surrounds the Trapezium cluster (Genzel \& Stutzki 1989; O'Dell 2001; Bally 2008; Goicoechea et al. 2019, 2020; Pabst et al. 2019, 2020). The Orion Bar is a prototypical strongly illuminated dense PDR. The impinging flux of stellar FUV photons $\left(G_{0}\right)$ is a few $10^{4}$ times the mean interstellar radiation field (Habing 1968). The Bar is seen nearly edge-on with respect to the FUV illuminating sources, mainly $\theta^{1}$ Ori $\mathrm{C}$, the most massive star in the Trapezium. This favorable orientation allows observers to spatially resolve the $\mathrm{H}^{+}$-to-H transition (the ionization front or IF; see, e.g., Walmsley et al. 2000; Pellegrini et al. 2009) from the H-to- $\mathrm{H}_{2}$ transition (the dissociation front or DF; see, e.g., Allers et al. 2005; van der Werf et al. 1996, 2013; Wyrowski et al. 1997; Cuadrado et al. 2019). It also allows one to study the stratification of different molecular species as a function of cloud depth (i.e., as the flux of FUV photons is attenuated; see, e.g., Tielens et al. 1993; van der Wiel et al. 2009; Habart et al. 2010; Goicoechea et al. 2016; Parikka et al. 2017; Andree-Labsch et al. 2017). 

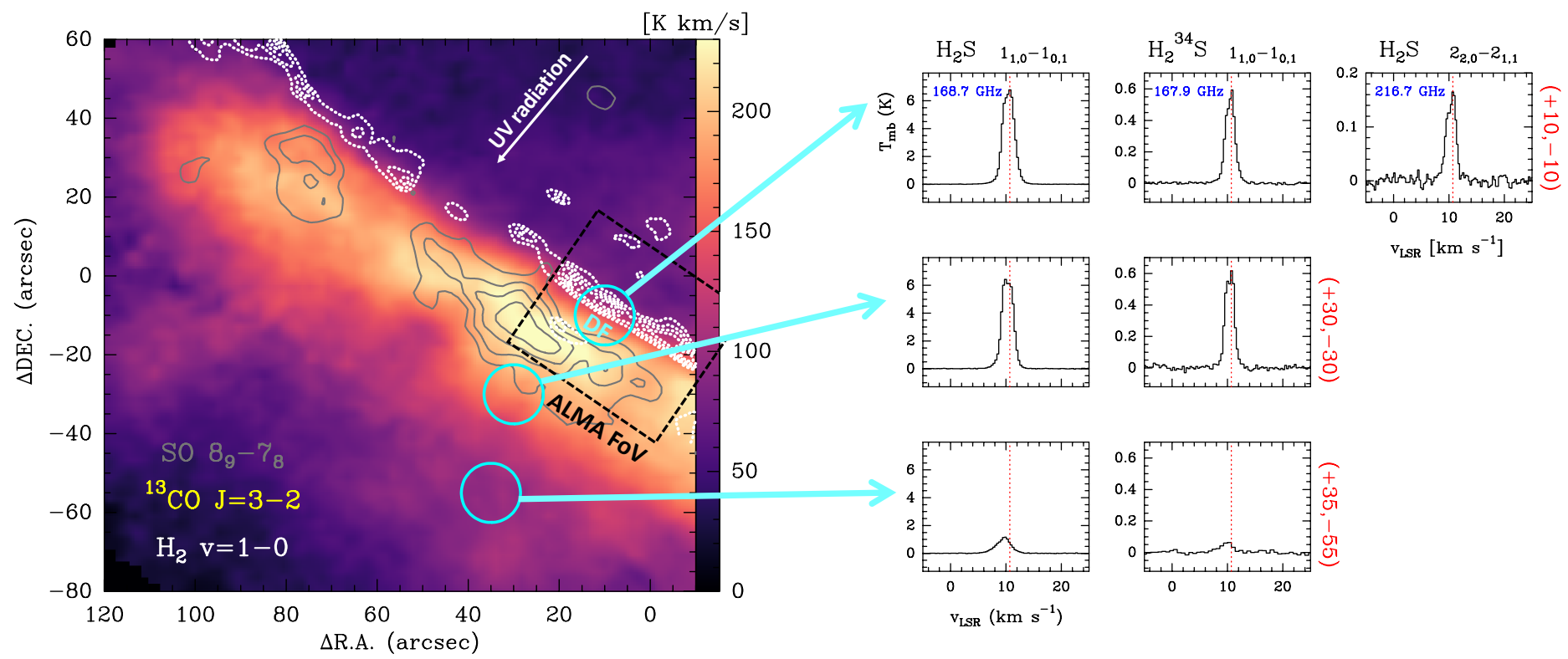

$\mathrm{v}_{\mathrm{LSR}}\left[\mathrm{km} \mathrm{s}^{-1}\right]$
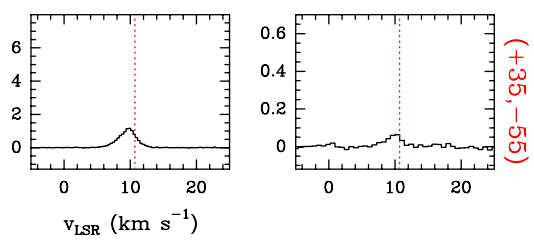

Fig. 1. Overview of the Orion Bar. The $\left(0^{\prime \prime}, 0^{\prime \prime}\right)$ position corresponds to $\alpha_{2000}=05^{\mathrm{h}} 35^{\mathrm{m}} 20.1^{\mathrm{s}} ; \delta_{2000}=-05^{\circ} 25^{\prime} 07.0^{\prime \prime}$. Left panel: Integrated line intensity maps in the ${ }^{13} \mathrm{CO} J=3-2$ (color scale) and SO $8_{9}-7_{8}$ emission (gray contours; from 6 to $23.5 \mathrm{~K} \mathrm{~km} \mathrm{~s}^{-1}$ in steps of $2.5 \mathrm{~K} \mathrm{~km} \mathrm{~s}^{-1}$ ) obtained with the IRAM $30 \mathrm{~m}$ telescope at $8^{\prime \prime}$ resolution. The white dotted contours delineate the position of the $\mathrm{H}_{2}$ dissociation front as traced by the infrared $\mathrm{H}_{2} v=1-0 \mathrm{~S}$ (1) line (from 1.5 to $4.0 \times 10^{-4} \mathrm{erg} \mathrm{s}^{-1} \mathrm{~cm}^{-2} \mathrm{sr}^{-1}$ in steps of $0.5 \times 10^{-4} \mathrm{erg} \mathrm{s}^{-1} \mathrm{~cm}^{-2} \mathrm{sr}^{-1}$; from Walmsley et al. 2000). The black-dashed rectangle shows the smaller FoV imaged with ALMA (Fig. 3). The DF position has been observed with SOFIA, IRAM $30 \mathrm{~m}$, and Herschel. Cyan circles represent the $\sim 15^{\prime \prime}$ beam at $168 \mathrm{GHz}$. Right panel: $\mathrm{H}_{2} \mathrm{~S}$ lines lines detected toward three positions of the Orion Bar.

Regarding sulfur ${ }^{2}$, several studies previously reported the detection of S-bearing molecules in the Orion Bar. These include $\mathrm{CS}, \mathrm{C}^{34} \mathrm{~S}, \mathrm{SO}, \mathrm{SO}_{2}$, and $\mathrm{H}_{2} \mathrm{~S}$ (Hogerheijde et al. 1995; Jansen et al. 1995), $\mathrm{SO}^{+}$(Fuente et al. 2003), $\mathrm{C}^{33} \mathrm{~S}, \mathrm{HCS}^{+}, \mathrm{H}_{2} \mathrm{CS}$, and NS (Leurini et al. 2006), and $\mathrm{SH}^{+}$(Nagy et al. 2013). These detections refer to modest angular resolution pointed observations using single-dish telescopes. Higher-angular-resolution interferometric imaging of $\mathrm{SH}^{+}$, SO, and $\mathrm{SO}^{+}$(Goicoechea et al. 2017) was possible thanks to the Atacama Compact Array (ACA).

\subsection{Observations of $\mathrm{H}_{2} \mathrm{~S}$ isotopologues and $\mathrm{H}_{3} \mathrm{~S}^{+}$}

We observed the Orion Bar with the IRAM $30 \mathrm{~m}$ telescope at Pico Veleta (Spain). We used the EMIR receivers in combination with the Fast Fourier Transform Spectrometer (FTS) backends at $200 \mathrm{kHz}$ resolution $\left(\sim 0.4 \mathrm{~km} \mathrm{~s}^{-1}, \sim 0.3 \mathrm{~km} \mathrm{~s}^{-1}\right.$, and $\sim 0.2 \mathrm{~km} \mathrm{~s}^{-1}$ at $\sim 168 \mathrm{GHz}, \sim 217 \mathrm{GHz}$, and $\sim 293 \mathrm{GHz}$, respectively). These observations are part of a complete line survey covering the frequency range $80-360 \mathrm{GHz}$ (Cuadrado et al. 2015, 2016, 2017, 2019) and include deep integrations at $168 \mathrm{GHz}$ toward three positions of the PDR located at a distance of $14^{\prime \prime}, 40^{\prime \prime}$, and $65^{\prime \prime}$ from the IF (see Fig. 1). Their offsets with respect to the IF position at $\alpha_{2000}=05^{\mathrm{h}} 35^{\mathrm{m}} 20.1^{\mathrm{s}}, \delta_{2000}=-05^{\circ} 25^{\prime} 07.0^{\prime \prime}$ are $\left(+10^{\prime \prime},-10^{\prime \prime}\right)$, $\left(+30^{\prime \prime},-30^{\prime \prime \prime}\right)$, and $\left(+35^{\prime \prime},-55^{\prime \prime}\right)$. The first position is the DF.

We carried out these observations in the position switching mode taking a distant reference position at $\left(-600^{\prime \prime}, 0^{\prime \prime}\right)$. The half power beam width (HPBW) at $\sim 168 \mathrm{GHz}, \sim 217 \mathrm{GHz}$, and $\sim 293 \mathrm{GHz}$ is $\sim 15^{\prime \prime}, \sim 11^{\prime \prime}$, and $\sim 8^{\prime \prime}$, respectively. The latest observations (those at $168 \mathrm{GHz}$ ) were performed in March 2020. The data were first calibrated in the antenna temperature scale $T_{\mathrm{A}}^{*}$ and then converted to the main beam temperature scale, $T_{\mathrm{mb}}$, using

\footnotetext{
2 Sulfur has four stable isotopes, in decreasing order of abundance: ${ }^{32} \mathrm{~S}\left(I_{\mathrm{N}}=0\right),{ }^{34} \mathrm{~S}\left(I_{\mathrm{N}}=0\right),{ }^{33} \mathrm{~S}\left(I_{\mathrm{N}}=3 / 2\right)$, and ${ }^{36} \mathrm{~S}\left(I_{\mathrm{N}}=0\right)$, where $I_{\mathrm{N}}$ is the nuclear spin. The most abundant isotope is here simply referred to as $\mathrm{S}$.
}

$T_{\mathrm{mb}}=T_{\mathrm{A}}^{*} / \eta_{\mathrm{mb}}$, where $\eta_{\mathrm{mb}}$ is the antenna efficiency $\left(\eta_{\mathrm{mb}}=0.74 \mathrm{at}\right.$ $\sim 168 \mathrm{GHz}$ ). We reduced and analyzed the data using the GILDAS software as described in Cuadrado et al. (2015). The typical rms noise of the spectra is $\sim 3.5,5.3$, and $7.8 \mathrm{mK}$ per velocity channel at $\sim 168 \mathrm{GHz}, \sim 217 \mathrm{GHz}$, and $\sim 293 \mathrm{GHz}$, respectively. Figures 1 and 2 show the detection of $o-\mathrm{H}_{2} \mathrm{~S}_{1,0}-1_{0,1}(168.7 \mathrm{GHz})$, $p-\mathrm{H}_{2} \mathrm{~S} 2_{2,0}-2_{1,1}(216.7 \mathrm{GHz})$, and $o-\mathrm{H}_{2}{ }^{34} \mathrm{~S}_{1,0}-1_{0,1}$ lines $(167.9 \mathrm{GHz})$ (see Table E.1 for the line parameters), as well as several $o-\mathrm{H}_{2}{ }^{33} \mathrm{~S} 1_{1,0}-1_{0,1}$ hyperfine lines $(168.3 \mathrm{GHz})$.

We complemented our dataset with higher frequency $\mathrm{H}_{2} \mathrm{~S}$ lines detected by the Herschel Space Observatory (Nagy et al. 2017) toward the " $\mathrm{CO}^{+}$peak" position (Stoerzer et al. 1995), which is located at only $\sim 4^{\prime \prime}$ from our DF position (i.e., within the HPBW of these observations). These observations were carried out with the HIFI receiver (de Graauw et al. 2010) at a spectralresolution of $1.1 \mathrm{MHz}\left(0.7 \mathrm{~km} \mathrm{~s}^{-1}\right.$ at $\left.500 \mathrm{GHz}\right)$. HIFI's HPBW range from $\sim 42^{\prime \prime}$ to $\sim 20^{\prime \prime}$ in the $500-1000 \mathrm{GHz}$ window (Roelfsema et al. 2012). The list of additional hydrogen sulfide lines detected by Herschel includes the $o-\mathrm{H}_{2} \mathrm{~S}_{2,1}-2_{1,2}(505.5 \mathrm{GHz})$, $2_{1,2}-1_{0,1}(736.0 \mathrm{GHz})$, and $3_{0,3}-2_{1,2}(993.1 \mathrm{GHz})$, as well as the $p-\mathrm{H}_{2} \mathrm{~S} 2_{0,2}-1_{1,1}(687.3 \mathrm{GHz})$ line. We used the line intensities, in the $T_{\mathrm{mb}}$ scale, shown in Table A.1 of Nagy et al. (2017).

In order to get a global view of the Orion Bar, we also obtained $2.5^{\prime} \times 2.5^{\prime}$ maps of the region observed by us with the IRAM $30 \mathrm{~m}$ telescope using the $330 \mathrm{GHz}$ EMIR receiver and the FTS backend at $200 \mathrm{kHz}$ spectral-resolution $\left(\sim 0.2 \mathrm{~km} \mathrm{~s}^{-1}\right)$. On-the-fly (OTF) scans were obtained along and perpendicular to the Bar. The resulting spectra were gridded to a data cube through convolution with a Gaussian kernel providing a final resolution of $\sim 8^{\prime \prime}$. The total integration time was $\sim 6 \mathrm{~h}$. The achieved rms noise is $\sim 1 \mathrm{~K}$ per resolution channel. Figure 1 shows the spatial distribution of the ${ }^{13} \mathrm{CO} J=3-2(330.5 \mathrm{GHz})$ and $\mathrm{SO} 8_{9}-7_{8}(346.5 \mathrm{GHz})$ integrated line intensities. 


\subsection{ALMA imaging of Orion Bar edge in $\mathrm{SH}^{+}$emission}

We carried out mosaics of a small field of the Orion Bar using twenty-seven ALMA 12 m antennas in band 7 (at $2346 \mathrm{GHz}$ ). These unpublished observations belong to project 2012.1.00352.S (P.I.: J. R. Goicoechea) and consisted of a 27-pointing mosaic centered at $\alpha(2000)=5^{h} 35^{m} 20.6^{s} ; \delta(2000)=-05^{\circ} 25^{\prime} 20^{\prime \prime}$. The total field-of-view (FoV) is $58^{\prime \prime} \times 52^{\prime \prime}$ (shown in Fig. 1). The two hyperfine line components of the $\mathrm{SH}^{+} N_{J}=1_{0}-0_{1}$ transition were observed with correlators providing $\sim 500 \mathrm{kHz}$ resolution $\left(0.4 \mathrm{~km} \mathrm{~s}^{-1}\right)$ over a $937.5 \mathrm{MHz}$ bandwidth. The total observation time with the ALMA $12 \mathrm{~m}$ array was $\sim 2 \mathrm{~h}$. In order to recover the large-scale extended emission filtered out by the interferometer, we used deep and fully sampled single-dish maps, obtained with the total-power (TP) antennas at $19^{\prime \prime}$ resolution, as zero- and short-spacings. Data calibration procedures and image synthesis steps are described in Goicoechea et al. (2016). The synthesized beam is $\sim 1^{\prime \prime}$. This is a factor of $\sim 4$ better than previous interferometric $\mathrm{SH}^{+}$observations (Goicoechea et al. 2017). Figure 3 shows the resulting image of the $\mathrm{SH}^{+} 1_{0}-0_{1} F=1 / 2-3 / 2$ hyperfine emission line at $345.944 \mathrm{GHz}$. We rotated this image $37.5^{\circ}$ clockwise to bring the FUV illumination in the horizontal direction. The typical rms noise of the final cube is $\sim 80 \mathrm{mK}$ per velocity channel and $1^{\prime \prime}$-beam. As expected from their Einstein coefficients, the other $F=1 / 2-1 / 2$ hyperfine line component at $345.858 \mathrm{GHz}$ is a factor of $\sim 2$ fainter (see Table E.2) and the resulting image has low signal-to-noise $(\mathrm{S} / \mathrm{N})$.

We complemented the $\mathrm{SH}^{+}$dataset with the higher frequency lines observed by HIFI (Nagy et al. 2013, 2017) at $\sim 526 \mathrm{GHz}$ and $\sim 683 \mathrm{GHz}$ (upper limit). These pointed observations have HPBWs of $\sim 41^{\prime \prime}$ and $\sim 32^{\prime \prime}$ respectively, thus they do not spatially resolve the $\mathrm{SH}^{+}$emission. To determine their beam coupling factors $\left(f_{\mathrm{b}}\right)$, we smoothed the bigger $4^{\prime \prime}$-resolution $\mathrm{ACA}+\mathrm{TP} \mathrm{SH}^{+}$image shown in Goicoechea et al. (2017) to the different HIFI's HPBWs. We obtain $f_{\mathrm{b}} \simeq 0.4$ at $\sim 526 \mathrm{GHz}$ and $f_{\mathrm{b}} \simeq 0.6$ at $\sim 683 \mathrm{GHz}$. The corrected intensities are computed as $W_{\text {corr }}=W_{\text {HIFI }} / f_{\mathrm{b}}$. These correction factors are only a factor of $\lesssim 2$ lower than simply assuming uniform $\mathrm{SH}^{+}$emission from a $10^{\prime \prime}$ width filament.

\subsection{SOFIA/GREAT search for SH emission}

We finally used the GREAT receiver (Heyminck et al. 2012) on board the Stratospheric Observatory For Infrared Astronomy (SOFIA; Young et al. 2012) to search for the lowestenergy rotational lines of $\mathrm{SH}\left({ }^{2} \Pi_{3 / 2} J=3 / 2-1 / 2\right)$ at 1382.910 and 1383.241 GHz (e.g., Klisch et al. 1996; Martin-Drumel et al. 2012). These lines lie in a frequency gap that Herschel/HIFI could not observe from space. These SOFIA observations belong to project 07_0115 (P.I.: J. R. Goicoechea). The SH lines were searched on the lower side band of 4GREAT band 3. We employed the 4GREAT/HFA frontends and 4GFFT spectrometers as backends. The HPBW of SOFIA at $1.3 \mathrm{THz}$ is $\sim 20^{\prime \prime}$, thus comparable with IRAM $30 \mathrm{~m} / \mathrm{EMIR}$ and Herschel/HIFI observations. We also employed the total power mode with a reference position at $\left(-600^{\prime \prime}, 0^{\prime \prime}\right)$. The original plan was to observe during two flights in November 2019 but due to bad weather conditions, only $\sim 70 \mathrm{~min}$ of observations were carried out in a single flight.

After calibration, data reduction included: removal of a first order spectral baseline, dropping scans with problematic receiver response, rms weighted average of the spectral scans, and calibration to $T_{\mathrm{mb}}$ intensity scale $\left(\eta_{\mathrm{mb}}=0.71\right)$. The final spectrum, smoothed to a velocity-resolution of $1 \mathrm{~km} \mathrm{~s}^{-1}$ has a rms noise of $\sim 50 \mathrm{mK}$ (shown in Fig. 4). Two emission peaks are seen at the

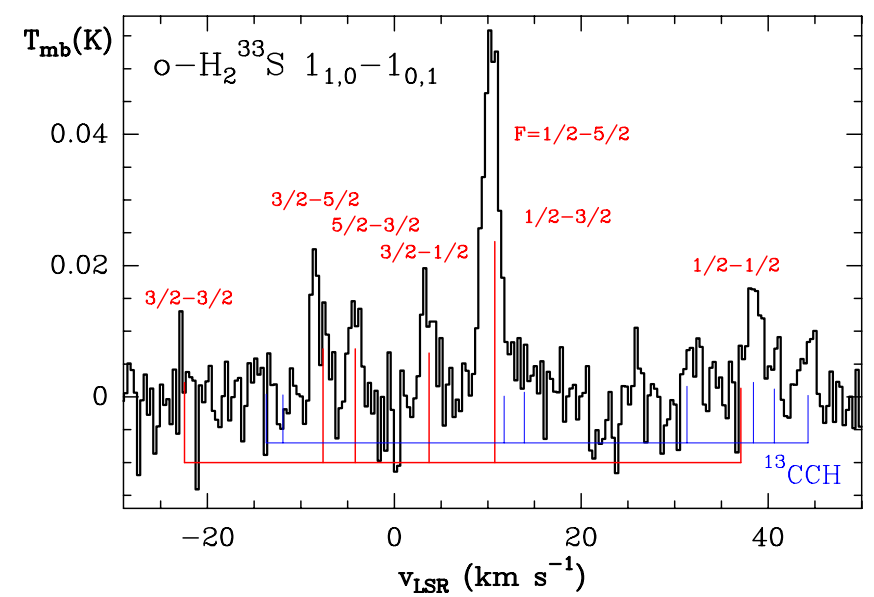

Fig. 2. Detection of $\mathrm{H}_{2}{ }^{33} \mathrm{~S}$ (at $\sim 168.3 \mathrm{GHz}$ ) toward the DF position of the Orion Bar. Red lines indicate hyperfine components. Blue lines show interloping lines from ${ }^{13} \mathrm{CCH}$. The length of each line is proportional to the transition line strength (taken from the Cologne Database for Molecular Spectroscopy, CDMS; Endres et al. 2016).

frequencies of the $\Lambda$-doublet lines. Unfortunately, the achieved rms is not enough to assure the unambiguous detection of each component of the doublet. Although the stacked spectrum does display a single line (suggesting a tentative detection) the resulting line-width $\left(\Delta v \simeq 7 \mathrm{~km} \mathrm{~s}^{-1}\right)$ is a factor of $\sim 3$ broader than expected in the Orion Bar (see Table E.3). Hence, this spectrum provides stringent upper limits to the $\mathrm{SH}$ column density but deeper integrations would be needed to confirm the detection.

\section{Observational results}

\section{1. $\mathrm{H}_{2}{ }^{32} \mathrm{~S}, \mathrm{H}_{2}{ }^{34} \mathrm{~S}$, and $\mathrm{H}_{2}{ }^{33} \mathrm{~S}$ across the PDR}

Figure 1 shows an expanded view of the Orion Bar in the ${ }^{13} \mathrm{CO}(J=3-2)$ emission. FUV radiation from the Trapezium stars comes from the upper-right corner of the image. The FUV radiation field is attenuated in the direction perpendicular to the Bar. The infrared $\mathrm{H}_{2} v=1-0 S$ (1) line emission (white contours) delineates the position of the $\mathrm{H}$-to- $\mathrm{H}_{2}$ transition, the DF. Many molecular species, such as SO, specifically emit from deeper inside the PDR where the flux of FUV photons has considerably decreased. In contrast, $\mathrm{H}_{2} \mathrm{~S}$, and even its isotopologue $\mathrm{H}_{2}^{34} \mathrm{~S}$, show bright $1_{1,0}-1_{0,1}$ line emission toward the DF (right panels in Fig. 1; see also Jansen et al. 1995). Rotationally excited $\mathrm{H}_{2} \mathrm{~S}$ lines have been also detected toward this position (Nagy et al. 2017), implying the presence of warm $\mathrm{H}_{2} \mathrm{~S}$ close to the irradiated cloud surface (i.e., at relatively low extinctions). The presence of moderately large $\mathrm{H}_{2} \mathrm{~S}$ column densities in the PDR is also demonstrated by the unexpected detection of the rare isotopologue $\mathrm{H}_{2}^{33} \mathrm{~S}$ toward the DF (at the correct LSR velocity of the PDR: $v_{\text {LSR }} \simeq 10.5 \mathrm{~km} \mathrm{~s}^{-1}$ ). Figure 2 shows the $\mathrm{H}_{2}^{33} \mathrm{~S} 1_{1,0}-1_{0,1}$ line and its hyperfine splittings (produced by the ${ }^{33} \mathrm{~S}$ nuclear spin). To our knowledge, $\mathrm{H}_{2}^{33} \mathrm{~S}$ lines had only been reported toward the hot cores in Sgr B2 and Orion KL before (Crockett et al. 2014).

The observed $o-\mathrm{H}_{2} \mathrm{~S} / o-\mathrm{H}_{2}^{34} \mathrm{~S} 1_{1,0}-1_{0,1}$ line intensity ratio toward the DF is $15 \pm 2$, below the solar isotopic ratio of ${ }^{32} \mathrm{~S} /{ }^{34} \mathrm{~S}=23$ (e.g., Anders \& Grevesse 1989). The observed ratio thus implies optically thick $o-\mathrm{H}_{2} \mathrm{~S}$ line emission at $\sim 168 \mathrm{GHz}$. However, the observed $o-\mathrm{H}_{2}^{34} \mathrm{~S} / o-\mathrm{H}_{2}^{33} \mathrm{~S} 1_{1,0}-1_{0,1}$ intensity ratio is $6 \pm 1$, thus compatible with the solar isotopic ratio $\left({ }^{34} \mathrm{~S} /{ }^{33} \mathrm{~S}=5.5\right)$ and with $\mathrm{H}_{2}^{34} \mathrm{~S}$ and $\mathrm{H}_{2}^{33} \mathrm{~S}$ optically thin emission. 
$\mathrm{CO} 3-2(345.8 \mathrm{GHz})$

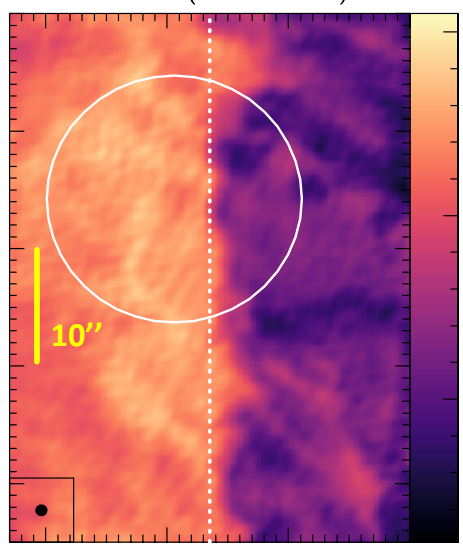

200

$\mathrm{SH}^{+} 1_{0}-0_{1}(345.9 \mathrm{GHz})$

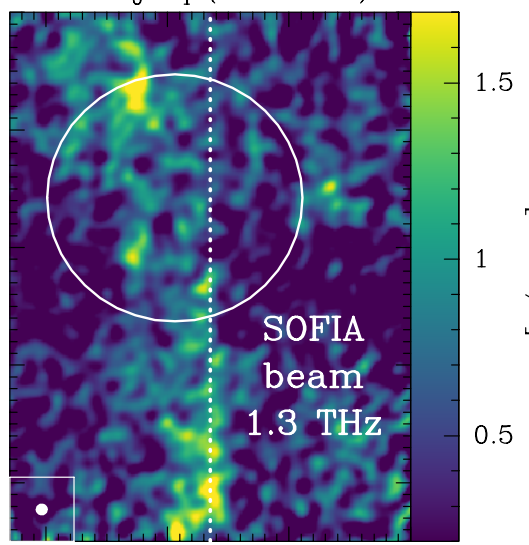

$\mathrm{H}_{2} \quad \mathrm{v}=1-0 \mathrm{~S}(1)(2.12 \mu \mathrm{m})$

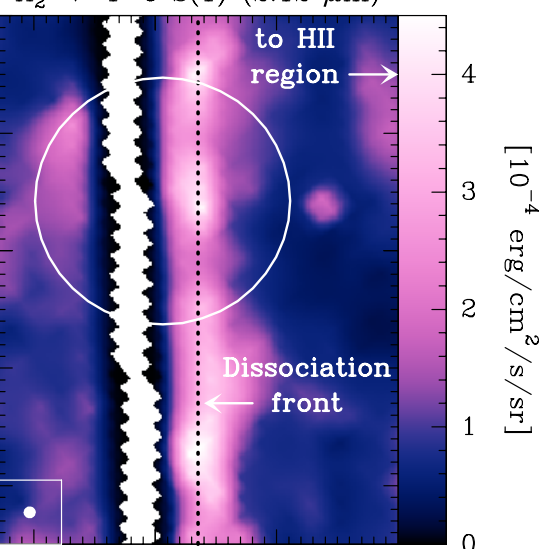

Fig. 3. ALMA $11^{\prime \prime}$-resolution images zooming into the edge of the Orion Bar in ${ }^{12} \mathrm{CO} 3-2$ (left panel, Goicoechea et al. 2016) and $\mathrm{SH}^{+} 1_{0}-0_{1} F=1 / 2-3 / 2$ line (middle panel, integrated line intensity). The right panel shows the $\mathrm{H}_{2} v=1-0 \mathrm{~S}$ (1) line (Walmsley et al. 2000). We rotated these images (all showing the same FoV) with respect to Fig. 1 to bring the FUV illuminating direction in the horizontal direction (from the right). The circle shows the DF position targeted with SOFIA in SH (20" beam) and with the IRAM $30 \mathrm{~m}$ telescope in $\mathrm{H}_{2} \mathrm{~S}^{\prime \prime}$ and $\mathrm{H}_{3} \mathrm{~S}^{+}$.

\section{2. $\mathrm{SH}^{+}$emission from the $P D R$ edge}

Figure 3 zooms into a small field of the Bar edge. The ALMA image of the $\mathrm{CO} J=3-2$ line peak temperature was first presented by Goicoechea et al. (2016). Because the $\mathrm{CO} J=3-2$ emission is nearly thermalized and optically thick from the DF to the molecular cloud interior, the line peak temperature scale $\left(T_{\text {peak }}\right)$ is a good proxy of the gas temperature $\left(T_{\mathrm{k}} \simeq T_{\mathrm{ex}} \simeq T_{\text {peak }}\right)$. The CO image implies small temperature variations around $T_{\mathrm{k}} \simeq 200 \mathrm{~K}$. The middle panel in Fig. 3 shows the ALMA image of the $\mathrm{SH}^{+}$ $N_{J}=1_{0}-0_{1} F=1 / 2-3 / 2$ hyperfine line at $345.944 \mathrm{GHz}$. Compared to $\mathrm{CO}$, the $\mathrm{SH}^{+}$emission follows the edge of the molecular PDR, akin to a filament of $\sim 10^{\prime \prime}$ width (for the spatial distribution of other molecular ions, see, Goicoechea et al. 2017). The $\mathrm{SH}^{+}$ emission shows localized small-scale emission peaks (density or column density enhancements) that match, or are very close to, the vibrationally excited $\mathrm{H}_{2}(v=1-0)$ emission (Fig. 3). We note that while some $\mathrm{H}_{2}(v=1-0)$ emission peaks likely coincide with gas density enhancements (e.g., Burton et al. 1990), the region also shows extended emission from FUV-pumped $\mathrm{H}_{2}(v=2-1)$ (van der Werf et al. 1996) that does not necessarily coincide with the $\mathrm{H}_{2}(v=1-0)$ emission peaks.

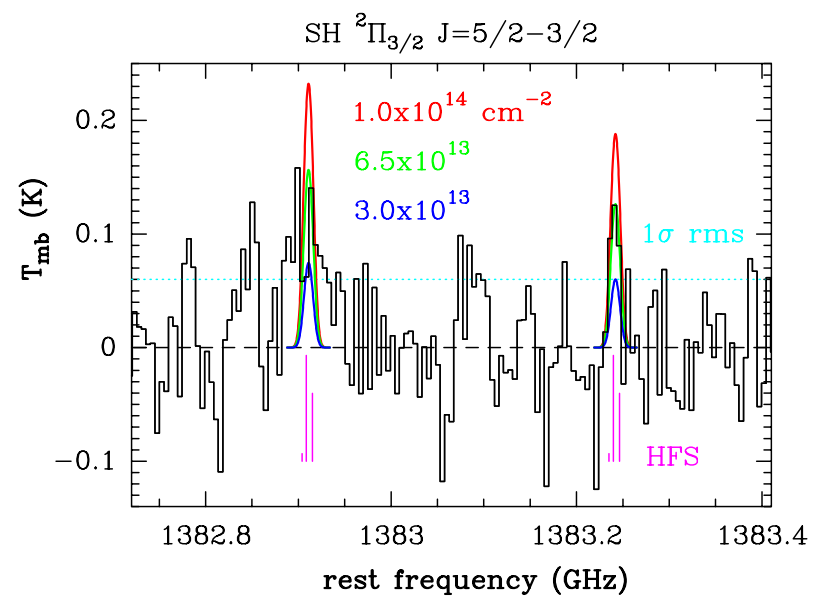

Fig. 4. Search for the $\mathrm{SH}^{2} \Pi_{3 / 2} J=5 / 2-3 / 2$ doublet (at $\sim 1383 \mathrm{GHz}$ ) toward the DF position with SOFIA/GREAT. Vertical magenta lines indicate the position of hyperfine splittings taken from CDMS.

\subsection{Search for $\mathrm{SH}, \mathrm{H}_{3} \mathrm{~S}^{+}$, and $\mathrm{H}_{2} \mathrm{~S} v_{2}=1$ emission}

We used SOFIA/GREAT to search for $\mathrm{SH}^{2} \Pi_{3 / 2} J=5 / 2-3 / 2$ lines toward the DF (Fig. 4). This would have been the first time that interstellar $\mathrm{SH}$ rotational lines were seen in emission. Unfortunately, the achieved rms of the observation does not allow a definitive confirmation of these lines, so here we will only discuss upper limits to the SH column density. The red, green, and blue curves in Fig. 4 show radiative transfer models for $n_{\mathrm{H}}=10^{6} \mathrm{~cm}^{-3}, T_{\mathrm{k}}=200 \mathrm{~K}$, and different $\mathrm{SH}$ column densities (see Sect. 4 for more details).

Our IRAM 30 m observations toward the DF neither resulted in a detection of $\mathrm{H}_{3} \mathrm{~S}^{+}$, a key gas-phase precursor of $\mathrm{H}_{2} \mathrm{~S}$. The $\sim 293.4 \mathrm{GHz}$ spectrum around the targeted $\mathrm{H}_{3} \mathrm{~S}^{+} 1_{0}-0_{0}$ line is shown in Fig. 5. Again, the achieved low rms allows us to provide a sensitive upper limit to the $\mathrm{H}_{3} \mathrm{~S}^{+}$column density. This results in $N\left(\mathrm{H}_{3} \mathrm{~S}^{+}\right)=(5.5-7.5) \times 10^{10} \mathrm{~cm}^{-2}(5 \sigma)$ assuming an excitation temperature range $T_{\mathrm{ex}}=10-30 \mathrm{~K}$ and extended emission. Given the bright $\mathrm{H}_{2} \mathrm{~S}$ emission close to the edge of the Orion Bar, and because $\mathrm{H}_{2} \mathrm{~S}$ formation at the DF might be driven by very exoergic processes, we also searched for the $1_{1,0}-1_{0,1}$ line of vibrationally excited $\mathrm{H}_{2} \mathrm{~S}$ (in the bending mode $v_{2}$ ). The frequency of this line lies at $\sim 181.4 \mathrm{GHz}$ (Azzam et al. 2013), thus at the end of our $2 \mathrm{~mm}$-band observations of the DF $(\mathrm{rms} \simeq 16 \mathrm{mK})$. However, we do not detect this line either.

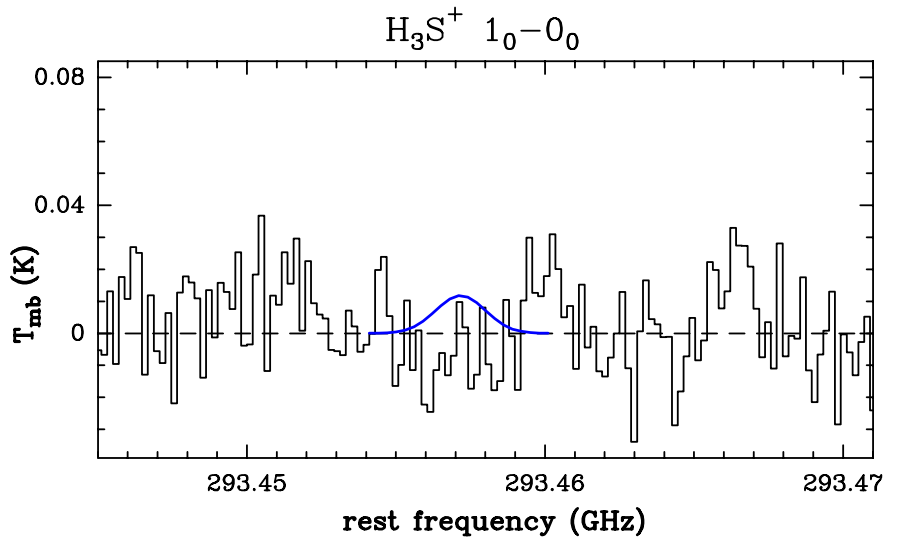

Fig. 5. Search for $\mathrm{H}_{3} \mathrm{~S}^{+}$toward the Orion Bar with the IRAM $30 \mathrm{~m}$ telescope. The blue curve shows the expected position of the line. 


\section{Coupled nonlocal excitation and chemistry}

In this section we study the rotational excitation of the observed $\mathrm{S}$-bearing hydrides ${ }^{3}$. We determine the $\mathrm{SH}^{+}, \mathrm{SH}$ (upper limit), and $\mathrm{H}_{2} \mathrm{~S}$ column densities in the Orion Bar, and the "average" gas physical conditions in the sense that we search for the combination of single $T_{\mathrm{k}}, n_{\mathrm{H}}$, and $N$ that better reproduces the observed line intensities (so-called "single-slab" approach). In Sect. 6 we expand these excitation models to multi-slab calculations that take into account the expected steep gradients in a PDR.

In the ISM, rotationally excited levels are typically populated by inelastic collisions. However, the lifetime of very reactive molecules can be so short that the details of their formation and destruction need to be taken into account when determining how these levels are actually populated (Black 1998). Reactive collisions (collisions that lead to a reaction and thus to molecule destruction) influence the excitation of these species when their timescales become comparable to those of nonreactive collisions. The lifetime of reactive molecular ions observed in PDRs (e.g., Fuente et al. 2003; Nagy et al. 2013; van der Tak et al. 2013; Goicoechea et al. 2017, 2019) can be so short that they do not get thermalized by nonreactive collisions or by absorption of the background radiation field (Black 1998). In these cases, a proper treatment of the molecule excitation requires including chemical formation and destruction rates in the statistical equilibrium equations $\left(\mathrm{d} n_{i} / \mathrm{d} t=0\right)$ that determine the level populations:

$$
\begin{aligned}
& \sum_{j>i} n_{j} A_{j i}+\sum_{j \neq i} n_{i}\left(B_{j i} \bar{J}_{j i}+C_{j i}\right)+F_{i}= \\
= & n_{i}\left(\sum_{j<i} A_{i j}+\sum_{j \neq i}\left(B_{i j} \bar{J}_{i j}+C_{i j}\right)+D_{i}\right),
\end{aligned}
$$

where $n_{i}\left[\mathrm{~cm}^{-3}\right]$ is the population of rotational level $i, A_{i j}$ and $B_{i j}$ are the Einstein coefficients for spontaneous and induced emission, $C_{i j}\left[\mathrm{~s}^{-1}\right]$ is the rate of inelastic collisions ${ }^{4}$ $\left(C_{i j}=\sum_{k} \gamma_{i j, k} n_{k}\right.$, where $\gamma_{i j, k}(T)\left[\mathrm{cm}^{3} \mathrm{~s}^{-1}\right]$ are the collisional rate coefficients and $k$ stands for $\mathrm{H}_{2}, \mathrm{H}$, and $e^{-}$), and $\bar{J}_{i j}$ is the mean intensity of the total radiation field over the line profile. In these equations, $n_{i} D_{i}$ is the destruction rate per unit volume of the molecule in level $i$, and $F_{i}$ its formation rate per unit volume (both in $\mathrm{cm}^{-3} \mathrm{~s}^{-1}$ ). When state-to-state formation rates are not available, and assuming that the destruction rate is the same in every level $\left(D_{i}=D\right)$, one can use the total destruction rate $D\left[\mathrm{~s}^{-1}\right]\left(=\sum_{k} n_{k} k_{k}(T)+\right.$ photodestruction rate, where $k_{k}\left[\mathrm{~cm}^{3} \mathrm{~s}^{-1}\right]$ is the state-averaged rate of the two-body chemical reaction with species $k$ ) and consider that the level populations of the nascent molecule follow a Boltzmann distribution at an effective formation temperature $T_{\text {form }}$ :

$$
F_{i}=F g_{i} e^{-E_{i} / k T_{\text {form }}} / Q\left(T_{\text {form }}\right) .
$$

In this formalism, $F\left[\mathrm{~cm}^{-3} \mathrm{~s}^{-1}\right]$ is the state-averaged formation rate per unit volume, $g_{i}$ the degeneracy of level $i$, and $Q\left(T_{\text {form }}\right)$ is the partition function at $T_{\text {form }}$ (van der Tak et al. 2007).

\footnotetext{
${ }^{3}$ Readers interested only in the chemistry of these species and in depthdependent PDR models could directly jump to Section 6.

4 We use the following inelastic collision rate coefficients $\gamma_{i j}$ :

- $\mathrm{SH}^{+}-e^{-}$, including hyperfine splittings (Hamilton et al. 2018).

- $\mathrm{SH}^{+}-o-\mathrm{H}_{2}$ and $p-\mathrm{H}_{2}$, including hyperfine splittings (Dagdigian 2019).

- $\mathrm{SH}^{+}-\mathrm{H}$, including hyperfine splittings (Lique et al. 2020).

- $o-\mathrm{H}_{2} \mathrm{~S}$ and $p-\mathrm{H}_{2} \mathrm{~S}$ with $o-\mathrm{H}_{2}$ and $p-\mathrm{H}_{2}$ (Dagdigian 2020).

- SH-He, including fine-structure splittings (Kłos et al. 2009).
}

This "formation pumping" formalism has been previously implemented in large velocity gradient codes to treat, for example, the local excitation of the very reactive ion $\mathrm{CH}^{+}(\mathrm{Nagy}$ et al. 2013; Godard \& Cernicharo 2013; Zanchet et al. 2013b; Faure et al. 2017). However, interstellar clouds are inhomogeneous and gas velocity gradients are typically modest at small spatial scales. This means that line photons can be absorbed and reemitted several times before leaving the cloud. Here we implemented this formalism in a Monte Carlo code that explicitly models the nonlocal behavior of the excitation and radiative transfer problem (see Appendix of Goicoechea et al. 2006).

Although radiative pumping by dust continuum photons does not generally dominate in PDRs, for completeness we also included radiative excitation by a modified blackbody at a dust temperature of $\sim 50 \mathrm{~K}$ and a dust opacity $\tau_{\lambda}=0.03(150 / \lambda[\mu \mathrm{m}])^{1.6}$ (which reproduces the observed intensity and wavelength dependence of the dust emission in the Bar; Arab et al. 2012). The molecular gas fraction, $f\left(\mathrm{H}_{2}\right)=2 n\left(\mathrm{H}_{2}\right) / n_{\mathrm{H}}$, is set to $2 / 3$, where $n_{\mathrm{H}}=n(\mathrm{H})+2 n\left(\mathrm{H}_{2}\right)$ is the total density of $\mathrm{H}$ nuclei. This choice is appropriate for the dissociation front and implies $n\left(\mathrm{H}_{2}\right)=n(\mathrm{H})$. As most electrons in the DF come from the ionization of carbon atoms, the electron density $n_{e}$ is set to $n_{e} \simeq n\left(\mathrm{C}^{+}\right)=1.4 \times 10^{-4} n_{\mathrm{H}}$ (e.g., Cuadrado et al. 2019). For the inelastic collisions with $o-\mathrm{H}_{2}$ and $p-\mathrm{H}_{2}$, we assumed that the $\mathrm{H}_{2}$ ortho-to-para (OTP) ratio is thermalized to the gas temperature.

\section{1. $\mathrm{SH}^{+}$excitation and column density}

We start by assuming that the main destruction pathway of $\mathrm{SH}^{+}$are reactions with $\mathrm{H}$ atoms and recombinations with electrons (see Sect. 6.1). Hence, the $\mathrm{SH}^{+}$destruction rate is $D \simeq n_{e} k_{\mathrm{e}}(T)+n(\mathrm{H}) k_{\mathrm{H}}(T)$ (see Table 1 for the relevant chemical destruction rates). For $T_{\mathrm{k}}=T_{\mathrm{e}}=200 \mathrm{~K}$ and $n_{\mathrm{H}}=10^{6} \mathrm{~cm}^{-3}$ (e.g., Goicoechea et al. 2016) this implies $D \simeq 10^{-4} \mathrm{~s}^{-1}$ (i.e., the lifetime of an $\mathrm{SH}^{+}$molecule in the Bar is less than $3 \mathrm{~h}$ ). At these temperatures and densities, $D$ is about ten times smaller than the rate of radiative and inelastic collisional transitions that depopulate the lowest-energy rotational levels of $\mathrm{SH}^{+}$. Hence, formation pumping does not significantly alter the excitation of the observed $\mathrm{SH}^{+}$lines, but it does influence the population of higher-energy levels. Formation pumping effects have been readily seen in $\mathrm{CH}^{+}$because this species is more reactive ${ }^{5}$ and its rotationally excited levels lie at higher-energy (i.e., their inelastic collision pumping rates are slower, e.g., Zanchet et al. 2013b)

Figure 6 shows results of several models: without formation pumping (dotted curves for model " $F=D=0$ "), adding formation pumping with $\mathrm{SH}^{+}$destruction by $\mathrm{H}$ and $e^{-}$(continuous curves for model " $F, D$ "), and using a factor of ten higher $\mathrm{SH}^{+}$destruction rates (simulating a dominant role of $\mathrm{SH}^{+}$photodissociation or destruction by reactions with vibrationally excited $\mathrm{H}_{2}$; dashed curves for model " $F, D \times 10$ "). Since the formation of $\mathrm{SH}^{+}$is driven by reaction (1) when $\mathrm{H}_{2}$ molecules are in $v \geq 2$, here we adopted $T_{\text {form }} \simeq E(v=2, J=0) / k-9860 \mathrm{~K} \approx 2000 \mathrm{~K}$. Because these are constant column density $N\left(\mathrm{SH}^{+}\right)$excitation and radiative transfer models, we used a normalized formation rate $F=\sum F_{i}$ that assumes steady-state $\mathrm{SH}^{+}$abundances consistent with the varying gas density in each model. That is, $F=\sum F_{i}=x\left(\mathrm{SH}^{+}\right) n_{\mathrm{H}} D$ $\left[\mathrm{cm}^{-3} \mathrm{~s}^{-1}\right]$, where $x$ refers to the abundance with respect to $\mathrm{H}$ nuclei.

\footnotetext{
${ }^{5} \mathrm{CH}^{+}$is more reactive than $\mathrm{SH}^{+}$because $\mathrm{CH}^{+}$does react with $\mathrm{H}_{2}(v=0)$ exothermically producing $\mathrm{CH}_{2}^{+}$at $k=1.2 \times 10^{-9} \mathrm{~cm}^{3} \mathrm{~s}^{-1}$ (Anicich 2003) and also because reaction of $\mathrm{CH}^{+}$with $\mathrm{H}$ is faster, $k=7.5 \times 10^{-10} \mathrm{~cm}^{3} \mathrm{~s}^{-1}$.
} 


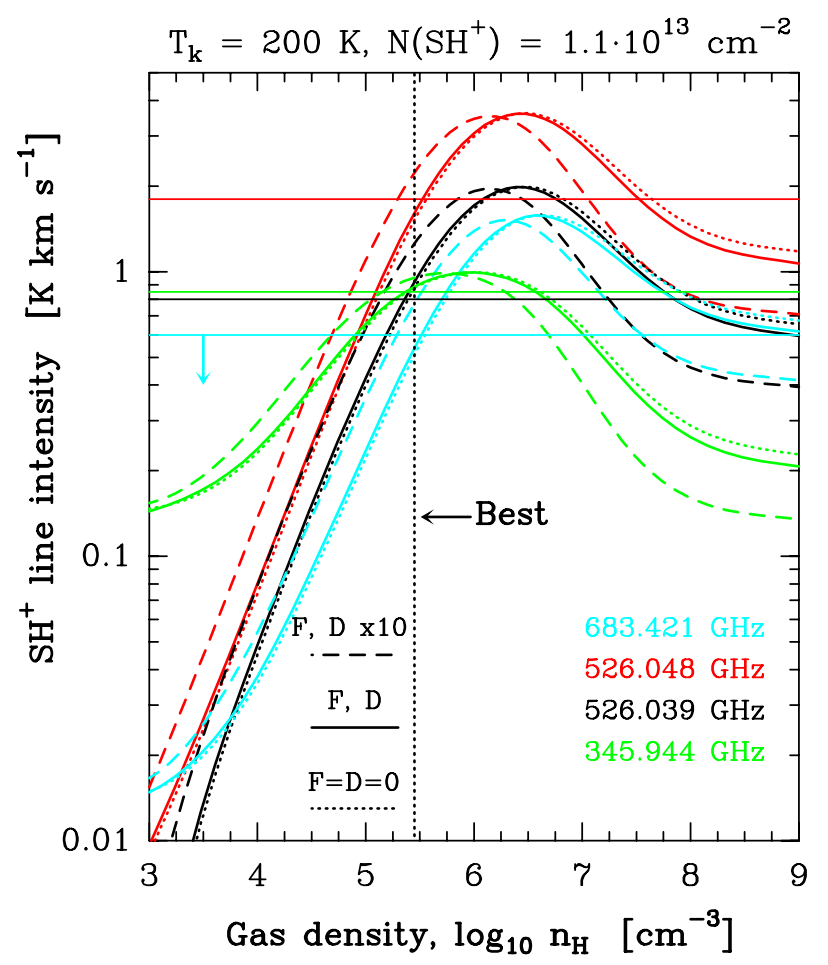

Fig. 6. Non-LTE excitation models of $\mathrm{SH}^{+}$. The horizontal lines mark the observed line intensities in the Orion Bar. Dotted curves are for a standard model $(F=D=0)$. Continuous curves are for a model that includes chemical destruction by $\mathrm{H}$ atoms and $e^{-}(\operatorname{model} F, D)$. Dashed lines are for a model in which destruction rates are multiplied by ten (model $F, D \times 10$ ). The vertical black line marks the best model.

The detected $\mathrm{SH}^{+}$rotational lines connect the fine-structure levels $N_{J}=1_{0}-0_{1}(345 \mathrm{GHz})$ and $1_{2}-0_{1}(526 \mathrm{GHz})$. Upper limits also exist for the $1_{1}-0_{1}(683 \mathrm{GHz})$ lines. $\mathrm{SH}^{+}$critical densities $\left(n_{\mathrm{cr}}=A_{i j} / \gamma_{i j}\right.$ ) for inelastic collisions with $\mathrm{H}$ or $\mathrm{H}_{2}$ are of the same order and equal to several $10^{6} \mathrm{~cm}^{-3}$. As for many molecular ions (e.g., Desrousseaux et al. 2021), $\mathrm{SH}^{+}-\mathrm{H}_{2}\left(\right.$ and $\left.\mathrm{SH}^{+}-\mathrm{H}\right)$ inelastic collisional rate coefficients ${ }^{4}$ are large $\left(\gamma_{i j} \gtrsim 10^{-10} \mathrm{~cm}^{3} \mathrm{~s}^{-1}\right)$. Thus, collisions with $\mathrm{H}$ (at low $A_{V}$ ) and $\mathrm{H}_{2}$ (at higher $A_{V}$ ) generally dominate over collisions with electrons $\left(\gamma_{i j}\right.$ of a few $\left.10^{-7} \mathrm{~cm}^{3} \mathrm{~s}^{-1}\right)$. At low densities (meaning $n_{\mathrm{H}}<n_{\mathrm{cr}}$ ) formation pumping increases the population of the higher-energy levels (and their $T_{\mathrm{ex}}$ ), but there are only minor effects in the low-energy submillimeter lines. At high densities, $n_{\mathrm{H}}>10^{7} \mathrm{~cm}^{-3}$, formation pumping with $T_{\text {form }}=2000 \mathrm{~K}$ produces lower intensities in these lines because the lowest-energy levels $\left(E_{\mathrm{u}} / k<T_{\mathrm{k}}<T_{\text {form }}\right)$ are less populated.

The best fit to the observed lines in model $\mathrm{F}, \mathrm{D}$ is for $N\left(\mathrm{SH}^{+}\right) \simeq 1.1 \times 10^{13} \mathrm{~cm}^{-3}, n_{\mathrm{H}} \simeq 3 \times 10^{5} \mathrm{~cm}^{-3}$, and $T_{\mathrm{k}} \simeq 200 \mathrm{~K}$. This is shown by the vertical dotted line in Fig. 6. This model is consistent with the upper limit intensity of the $683 \mathrm{GHz}$ line (Nagy et al. 2013). In this comparison, and following the morphology of the $\mathrm{SH}^{+}$emission revealed by ALMA (Fig. 3), we corrected the line intensities of the $\mathrm{SH}^{+}$ lines detected by Herschel/HIFI with the beam coupling factors discussed in Sec. 2.3, The observed $1_{2}-0_{1} / 1_{0}-0_{1}$ line ratio $(R=W(526.048) / W(345.944) \simeq 2)$ is sensitive to the gas density. In these models, $R$ is 1.1 for $n_{\mathrm{H}}=10^{5} \mathrm{~cm}^{-3}$ and 3.0 for $n_{\mathrm{H}}=10^{6} \mathrm{~cm}^{-3}$. We note that $n_{\mathrm{H}}$ could be lower if $\mathrm{SH}^{+}$formation/destruction rates were faster, as in the $F, D \times 10$ model. This could happen if $\mathrm{SH}^{+}$photodissociation or destruction reactions with $\mathrm{H}_{2}(v \geq 2)$ were faster than reactions of $\mathrm{SH}^{+}$with $\mathrm{H}$ atoms or with electrons. In Sec. 6 we show that this is not the case.

\subsection{SH excitation and column density}

$\mathrm{SH}$ is a ${ }^{2} \Pi$ open-shell radical with fine-structure, $\Lambda$-doubling, and hyperfine splittings (e.g., Martin-Drumel et al. 2012). However, the frequency separation of the $\mathrm{SH}^{2} \Pi_{3 / 2} J=5 / 2-3 / 2$ hyperfine components is too small to be spectrally resolved in observations of the Orion Bar (see Fig. 4). The available rate coefficients for inelastic collisions of $\mathrm{SH}$ with helium atoms do not resolve the hyperfine splittings. Hence, we first determined line frequencies, level degeneracies, and Einstein coefficients of an $\mathrm{SH}$ molecule without hyperfine structure. To do this, we took the complete set of hyperfine levels tabulated in CDMS. Lacking specific inelastic collision rate coefficients, we scaled the available $\mathrm{SH}-\mathrm{He}$ rates of Kłos et al. (2009) by the square root of the reduced mass ratios and estimated the $\mathrm{SH}-\mathrm{H}$ and $\mathrm{SH}-\mathrm{H}_{2}$ collisional rates.

The scaled rate coefficients are about an order of magnitude smaller than those of $\mathrm{SH}^{+}$. However, the chemical destruction rate of $\mathrm{SH}$ at the PDR edge (reactions with $\mathrm{H}$, photodissociation, and photoionization, see Sect. 6.1) is also slower (we take the rates of $\mathrm{SH}-\mathrm{H}$ reactive collisions from Zanchet et al. 2019). We determine $D \simeq 3 \times 10^{-6} \mathrm{~s}^{-1}$ for $n_{\mathrm{H}}=10^{6} \mathrm{~cm}^{-3}$, $T_{\mathrm{k}}=200 \mathrm{~K}$, and $A_{V} \simeq 0.7 \mathrm{mag}$. Models in Fig. 7 include these chemical rates for $T_{\text {form }}=T_{\mathrm{k}}$ (a lower limit to the unknown formation temperature). Formation pumping enhances the intensity of the ${ }^{2} \Pi_{3 / 2} J=5 / 2-3 / 2$ ground-state lines by a few percent only.

To estimate the SH column density in the Orion Bar we compare with the upper limit intensities of the $\mathrm{SH}$ lines targeted by SOFIA. If $\mathrm{SH}$ and $\mathrm{SH}^{+}$arise from roughly the same gas at similar physical conditions $\left(n_{\mathrm{H}} \simeq 10^{6} \mathrm{~cm}^{-3}\right.$ and $\left.T_{k} \simeq 200 \mathrm{~K}\right)$ the best model column density is for $N(\mathrm{SH}) \leq(0.6-1.6) \times 10^{14} \mathrm{~cm}^{-2}$. If densities were lower, around $n_{\mathrm{H}} \simeq 10^{5} \mathrm{~cm}^{-3}$, the upper limit $N(\mathrm{SH})$ column densities will be a factor ten higher.

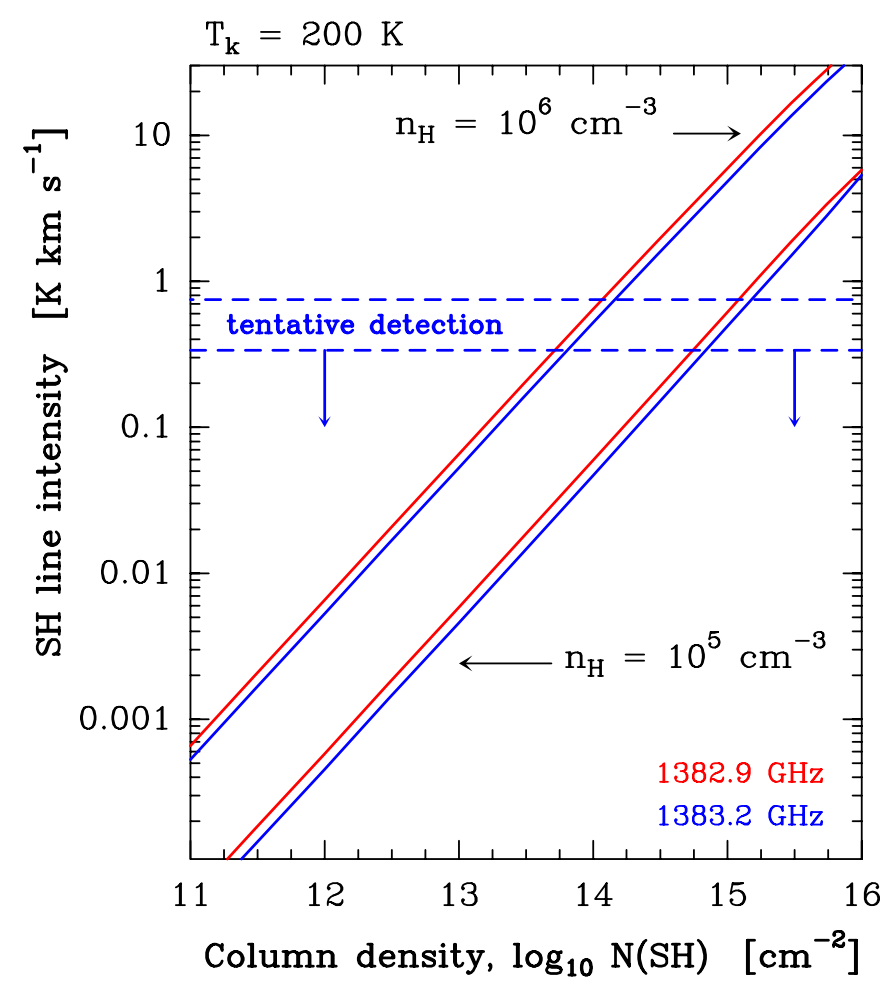

Fig. 7. Non-LTE excitation models of SH emission lines targeted with SOFIA/GREAT. Horizontal dashed lines refer to observational limits, assuming extended emission (lower intensities) and for a 10" width emission filament at the PDR surface (higher intensities). 

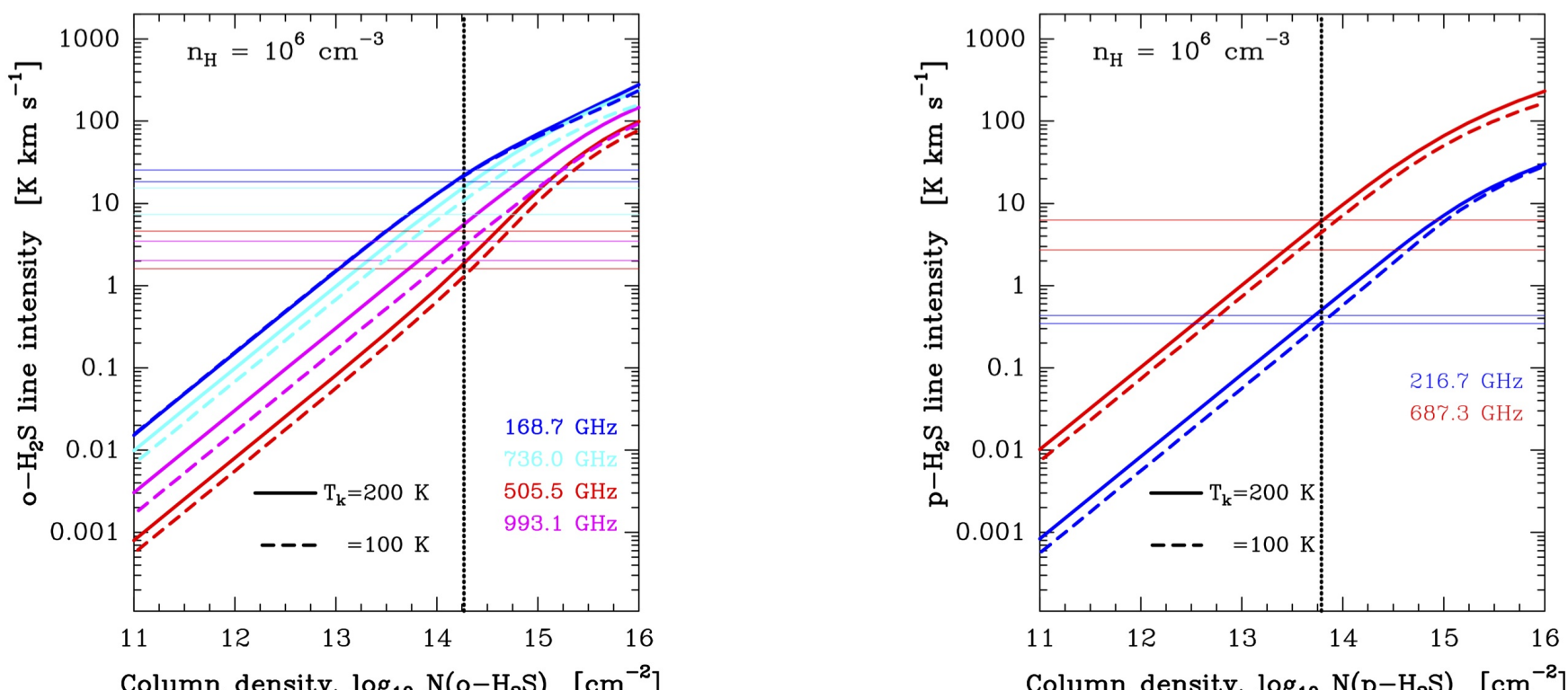

Column density, $\log _{10} \mathrm{~N}\left(\mathrm{p}-\mathrm{H}_{2} \mathrm{~S}\right) \quad\left[\mathrm{cm}^{-2}\right]$

Fig. 8. Non-LTE excitation models for $o-\mathrm{H}_{2} \mathrm{~S}$ and $p-\mathrm{H}_{2} \mathrm{~S}$. Thin horizontal lines show the observed intensities assuming either extended emission (lower limit) or emission that fills the $15^{\prime \prime}$ beam at $168.7 \mathrm{GHz}$. The vertical line marks the best model, resulting in an OTP ratio of $2.9 \pm 0.3$.

\section{3. $\mathrm{H}_{2} \mathrm{~S}$ excitation and column density}

$\mathrm{H}_{2} \mathrm{~S}$ has a $X^{2} A$ ground electronic state and two nuclear spin symmetries that we treat separately, $o-\mathrm{H}_{2} \mathrm{~S}$ and $p-\mathrm{H}_{2} \mathrm{~S}$. Previous studies of the $\mathrm{H}_{2} \mathrm{~S}$ line excitation have used collisional rates coefficients scaled from those of the $\mathrm{H}_{2} \mathrm{O}-\mathrm{H}_{2}$ system. Dagdigian (2020) recently carried out specific calculations of the cross sections of $o-\mathrm{H}_{2} \mathrm{~S}$ and $p-\mathrm{H}_{2} \mathrm{~S}$ inelastic collisions with $o-\mathrm{H}_{2}$ and $p-\mathrm{H}_{2}$ at different temperatures. The behavior of the new and the scaled rates is different and it depends on the $\mathrm{H}_{2}$ OTP ratio (e.g., on gas temperature) because the collisional cross sections are different for $o-\mathrm{H}_{2}-\mathrm{H}_{2} \mathrm{~S}$ and $p-\mathrm{H}_{2}-\mathrm{H}_{2} \mathrm{~S}$ systems. At the warm temperatures of the PDR, collisions with $o-\mathrm{H}_{2}$ dominate, resulting in rate coefficients for the $\sim 168 \mathrm{GHz} o-\mathrm{H}_{2} \mathrm{~S}$ line that are a factor up to $\sim 2.5$ smaller than those scaled from $\mathrm{H}_{2} \mathrm{O}-\mathrm{H}_{2}$.

$\mathrm{H}_{2} \mathrm{~S}$ is not a reactive molecule. At the edge of the PDR its destruction is driven by photodissociation. We determine that the radiative and collisional pumping rates are typically a factor of $\sim 100$ higher than $D \approx 2 \times 10^{-6} \mathrm{~s}^{-1}$ (for $n_{\mathrm{H}}=10^{6} \mathrm{~cm}^{-3}, T_{\mathrm{k}}=200 \mathrm{~K}$, $G_{0} \simeq 10^{4}$, and $A_{V} \simeq 0.7 \mathrm{mag}$ ). Figure 8 shows non-LTE $o-\mathrm{H}_{2} \mathrm{~S}$ and $p-\mathrm{H}_{2} \mathrm{~S}$ excitation and radiative transfer models. $\mathrm{As}_{2} \mathrm{~S}$ may have its abundance peak deeper inside the PDR and display more extended emission than $\mathrm{SH}^{+}$(e.g., Sternberg \& Dalgarno 1995), we show results for $T_{\mathrm{k}}=200$ and $100 \mathrm{~K}$. When comparing with the observed line intensities, we considered either emission that fills all beams, or a correction that assumes that the $\mathrm{H}_{2} \mathrm{~S}$ emission only fills the $15^{\prime \prime}$ beam of the IRAM $30 \mathrm{~m}$ telescope at $168 \mathrm{GHz}$. The vertical dotted lines in Fig. 8 show the best model, $N\left(\mathrm{H}_{2} \mathrm{~S}\right)=N\left(o-\mathrm{H}_{2} \mathrm{~S}\right)+N\left(p-\mathrm{H}_{2} \mathrm{~S}\right)=2.5 \times 10^{14} \mathrm{~cm}^{-2}$, with an OTP ratio of $2.9 \pm 0.3$, thus consistent with the high-temperature statistical ratio of 3/1 (see discussion at the end of Sect. 6.4). Models with lower densities, $n_{\mathrm{H}} \simeq 10^{5} \mathrm{~cm}^{-3}$, show worse agreement, and would translate into even higher $N\left(\mathrm{H}_{2} \mathrm{~S}\right)$ of $\gtrsim 10^{15} \mathrm{~cm}^{-2}$. In either case, these calculations imply large columns of warm $\mathrm{H}_{2} \mathrm{~S}$ toward the PDR. They result in a limit to the $\mathrm{SH}$ to $\mathrm{H}_{2} \mathrm{~S}$ column density ratio of $\leq 0.2-0.6$. This upper limit is already lower than the $N(\mathrm{SH}) / N\left(\mathrm{H}_{2} \mathrm{~S}\right)=1.1-3.0$ ratios observed in diffuse clouds (Neufeld et al. 2015). This difference suggests an enhanced $\mathrm{H}_{2} \mathrm{~S}$ formation mechanism in FUV-illuminated dense gas.

\section{New results on sulfur-hydride reactions}

In this section we summarize the ab initio quantum calculations we carried out to determine the vibrationally-state-dependent rates of gas-phase reactions of $\mathrm{H}_{2}(v>0)$ with several S-bearing species. We recall that all hydrogen abstraction reactions,

$\mathrm{S}^{+} \underset{(1)}{\stackrel{+\mathrm{H}_{2}}{\longrightarrow}} \mathrm{SH}^{+} \underset{(2)}{\stackrel{+\mathrm{H}_{2}}{\longrightarrow}} \mathrm{H}_{2} \mathrm{~S}^{+} \underset{(3)}{\stackrel{+\mathrm{H}_{2}}{\longrightarrow}} \mathrm{H}_{3} \mathrm{~S}^{+}, \quad \mathrm{S} \underset{(4)}{\stackrel{+\mathrm{H}_{2}}{\longrightarrow}} \mathrm{SH}$,

are very endoergic for $\mathrm{H}_{2}(v=0)$, with endothermicities in Kelvin units that are significantly higher than $T_{\mathrm{k}}$ even in PDRs. This is markedly different to $\mathrm{O}^{+}$chemistry, for which all hydrogen abstraction reactions leading to $\mathrm{H}_{3} \mathrm{O}^{+}$are exothermic and fast (Gerin et al. 2010; Neufeld et al. 2010; Hollenbach et al. 2012).

The endothermicity of reactions involving $\mathrm{H}_{n} \mathrm{~S}^{+}$ions decreases as the number of hydrogen atoms increases. The potential energy surfaces (PES) of these reactions possess shallow wells at the entrance and products channels (shown in Fig. 9). In addition, these PESs show saddle points between the energy walls of reactants and products whose heights increase with the number of $\mathrm{H}$ atoms. For reaction (2), the saddle point has an energy of $0.6 \mathrm{eV}$ $(\simeq 7,000 \mathrm{~K})$ and is slightly below the energy of the products. However, for reaction (3), the saddle point is above the energy of the products and is a reaction barrier. These saddle points act as a bottleneck in the gas-phase hydrogenation of $\mathrm{S}^{+}$.

If one considers the state dependent reactivity of vibrationally excited $\mathrm{H}_{2}$, the formation of $\mathrm{SH}^{+}$through reaction (1) becomes exoergic ${ }^{6}$ when $v \geq 2$ (Zanchet et al. 2019). The detection of bright $\mathrm{H}_{2} \mathrm{~S}$ emission in the Orion Bar (Figs. 1 and 4) might suggest that subsequent hydrogen abstraction reactions with $\mathrm{H}_{2}(v \geq 2)$ proceed as well. Motivated by these findings, and before carrying out any PDR model, we studied reaction (2) and the reverse process in detail. This required to build a full dimensional quantum PES of the $\mathrm{H}_{3} \mathrm{~S}^{+}\left(\mathrm{X}^{1} \mathrm{~A}_{1}\right)$ system (see Appendix A).

In addition, we studied reaction (4) (and its reverse) through quantum calculations. Details of these ab initio calculations and of the resulting reactive cross sections are given in Appendix B.

${ }_{6}$ If one considers $\mathrm{H}_{2}$ rovibrational levels, reaction (1) becomes exoergic for $v=0, J \geq 11$ and for $v=1, J \geq 7$ (Zanchet et al. 2019). 

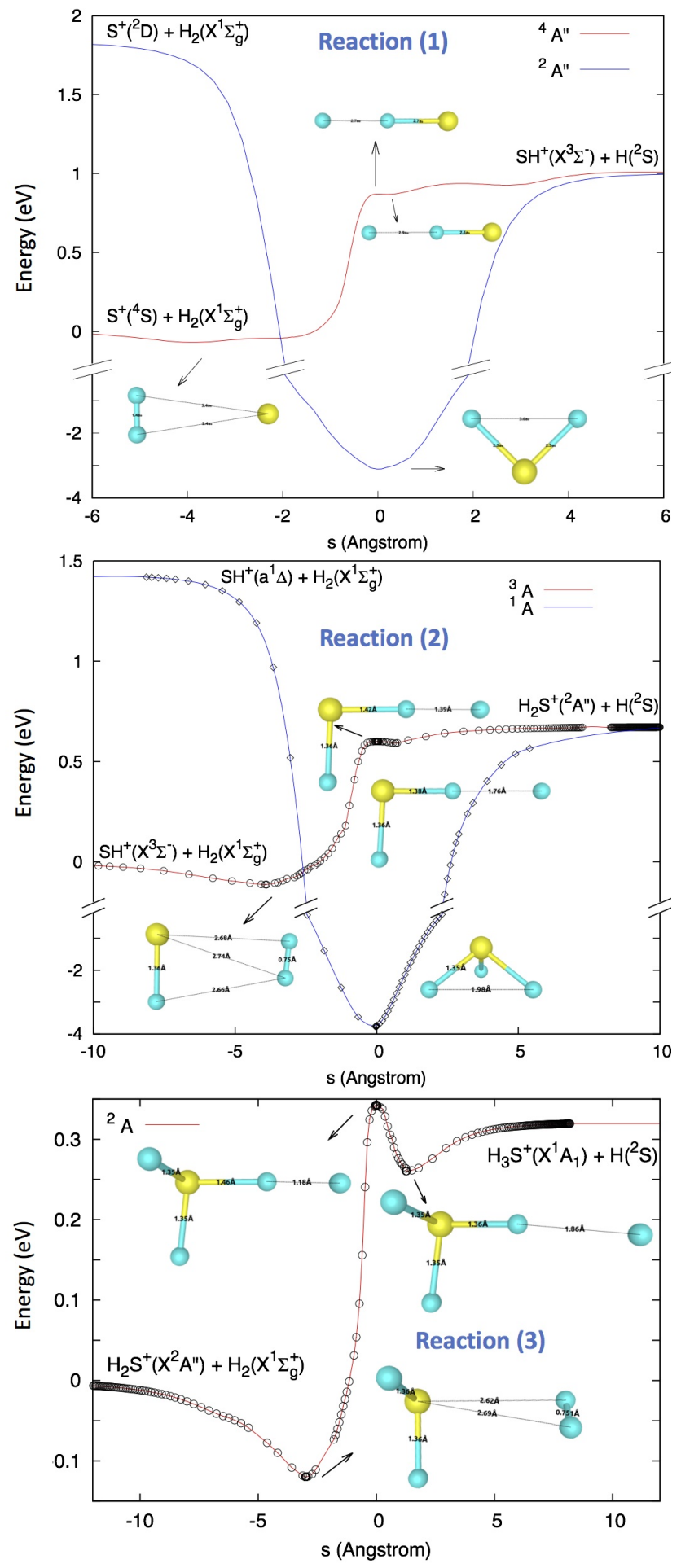

Fig. 9. Minimum energy paths for reactions (1), (2), and (3). Points correspond to RCCSD(T)-F12a calculations and lines to fits (Appendix A). The reaction coordinate, $s$, is defined independently for each path. The geometries of each species at $s=0$ are different.

Table 1 summarizes the updated reaction rate coefficients that we will include later in our PDR models.

The $\mathrm{H}_{2} \mathrm{~S}^{+}$formation rate through reaction (2) with $\mathrm{H}_{2}(v=0)$ is very slow. For $\mathrm{H}_{2}(v=1)$, the rate constant increases at $\approx 500 \mathrm{~K}$, corresponding to the opening of the $\mathrm{H}_{2} \mathrm{~S}^{+}+\mathrm{H}$ threshold. For $\mathrm{H}_{2}(v=2)$ and $\mathrm{H}_{2}(v=3)$, the reaction rate is much faster, close to the Langevin limit (see Appendix A.2). However, our estimated vibrational-state specific rates for $\mathrm{SH}$ forma-
Table 1. Relevant rate coefficients from a fit of the Arrhenius-like form $k(T)=\alpha(T / 300 \mathrm{~K})^{\beta} \exp (-\gamma / T)$ to the calculated reaction rates.

\begin{tabular}{lccr}
\hline \hline Reaction & $\begin{array}{c}\alpha \\
\left(\mathrm{cm}^{3} \mathrm{~s}^{-1}\right)\end{array}$ & $\beta$ & $\begin{array}{r}\gamma \\
(\mathrm{K})\end{array}$ \\
\hline $\mathrm{SH}^{+}+\mathrm{H}_{2}(v=1) \rightarrow \mathrm{H}_{2} \mathrm{~S}^{+}+\mathrm{H}$ & $4.97 \mathrm{e}-11$ & 0 & $1973.4^{a}$ \\
$\mathrm{SH}^{+}+\mathrm{H}_{2}(v=2) \rightarrow \mathrm{H}_{2} \mathrm{~S}^{+}+\mathrm{H}$ & $5.31 \mathrm{e}-10$ & -0.17 & $0^{a}$ \\
$\mathrm{SH}^{+}+\mathrm{H}_{2}(v=3) \rightarrow \mathrm{H}_{2} \mathrm{~S}^{+}+\mathrm{H}$ & $9.40 \mathrm{e}-10$ & -0.16 & $0^{a}$ \\
\hline $\mathrm{SH}^{+}+\mathrm{H} \rightarrow \mathrm{S}^{+}+\mathrm{H}_{2}$ & $1.86 \mathrm{e}-10$ & -0.41 & $27.3^{b}$ \\
\hline $\mathrm{SH}^{+}+e^{-} \rightarrow \mathrm{S}+\mathrm{H}$ & $2.00 \mathrm{e}-07$ & -0.50 & ${ }^{c}$ \\
\hline $\mathrm{H}_{2} \mathrm{~S}^{+}+\mathrm{H} \rightarrow \mathrm{SH}^{+}+\mathrm{H}_{2}$ & $6.15 \mathrm{e}-10$ & -0.34 & $0^{a}$ \\
\hline $\mathrm{S}+\mathrm{H}_{2}(v=2) \rightarrow \mathrm{SH}+\mathrm{H}$ & $\sim 8.6 \mathrm{e}-13$ & $\sim 2.3$ & $\sim 2500^{a}$ \\
$\mathrm{~S}+\mathrm{H}_{2}(v=3) \rightarrow \mathrm{SH}+\mathrm{H}$ & $\sim 1.7 \mathrm{e}-12$ & $\sim 2.0$ & $\sim 1500^{a}$ \\
\hline $\mathrm{SH}+\mathrm{H} \rightarrow \mathrm{S}+\mathrm{H}_{2}$ & $5.7 \mathrm{e}-13$ & 2.48 & $1600^{a, \dagger}$ \\
& $7.7 \mathrm{e}-14$ & 0.39 & $-1.3^{a, \dagger}$ \\
\hline $\mathrm{S}^{+}+\mathrm{H}_{2}(v=2) \rightarrow \mathrm{SH}^{+}+\mathrm{H}$ & $2.88 \mathrm{e}-10$ & -0.15 & $42.9^{b}$ \\
$\mathrm{~S}^{+}+\mathrm{H}_{2}(v=3) \rightarrow \mathrm{SH}^{+}+\mathrm{H}$ & $9.03 \mathrm{e}-10$ & -0.11 & $26.2^{b}$ \\
$\mathrm{~S}^{+}+\mathrm{H}_{2}(v=4) \rightarrow \mathrm{SH}^{+}+\mathrm{H}$ & $1.30 \mathrm{e}-09$ & -0.04 & $40.8^{b}$ \\
$\mathrm{~S}^{+}+\mathrm{H}_{2}(v=5) \rightarrow \mathrm{SH}^{+}+\mathrm{H}$ & $1.21 \mathrm{e}-09$ & 0.09 & $34.5^{b}$ \\
\hline
\end{tabular}

Notes. ${ }^{(a)}$ This work. ${ }^{(b)}$ From Zanchet et al. (2019). ${ }^{(c)}$ From Prasad \& Huntress (1980). ${ }^{\dagger}$ Total rate is the sum of the two expressions.

tion through reaction (4) $\left(\mathrm{S}+\mathrm{H}_{2}\right)$ are considerably smaller than for reactions (1) and (2), and show an energy barrier even for $\mathrm{H}_{2}(v=2)$ and $\mathrm{H}_{2}(v=3)$. We anticipate that this reaction is not a relevant formation route for $\mathrm{SH}$.

In FUV-illuminated environments, collisions with $\mathrm{H}$ atoms are very important because they compete with electron recombinations in destroying molecular ions, and also they contribute to their excitation. An important result of our calculations is that the destruction rate of $\mathrm{H}_{2} \mathrm{~S}^{+}\left(\mathrm{SH}^{+}\right)$in reactions with $\mathrm{H}$ atoms are a factor of $\geq 3.5(\geq 1.7)$ faster (at $\left.T_{\mathrm{k}} \leq 200 \mathrm{~K}\right)$ than those previously used in astrochemical models (Millar et al. 1986). Conversely, we find that destruction of $\mathrm{SH}$ in reactions with $\mathrm{H}$ atoms (Appendix B) is slower than previously assumed.

\section{PDR models of S-bearing hydrides}

We now investigate the chemistry of S-bearing hydrides and the effect of the new reaction rates in PDR models adapted to the Orion Bar conditions. In this analysis we used version 1.5.4. of the Meudon PDR code (Le Petit et al. 2006; Bron et al. 2014). Following our previous studies, we model the Orion Bar as a stationary PDR at constant thermal-pressure (i.e., with density and temperature gradients). When compared to time-dependent hydrodynamic PDR models (e.g., Hosokawa \& Inutsuka 2006; Bron et al. 2018; Kirsanova \& Wiebe 2019), stationary isobaric models seem a good description of the most exposed and compressed gas layers of the $\mathrm{PDR}$, from $A_{V} \approx 0.5$ to $\approx 5 \mathrm{mag}$ (Goicoechea et al. 2016; Joblin et al. 2018).

In our models, the FUV radiation field incident at the PDR edge is $G_{0}=2 \times 10^{4}$ (e.g., Marconi et al. 1998). We adopted an extinction to color-index ratio, $R_{V}=A_{V} / E_{B-V}$, of 5.5 (Joblin et al. 2018), consistent with the flatter extinction curve observed in Orion (Lee 1968; Cardelli et al. 1989). This choice implies slightly more penetration of FUV radiation into the cloud (e.g., Goicoechea \& Le Bourlot 2007). The main input parameters and elemental abundances of these PDR models are summarized in Table 2. Figure 10 shows the resulting $\mathrm{H}_{2}, \mathrm{H}$, and electron density profiles, as well as the $T_{\mathrm{k}}$ and $T_{\mathrm{d}}$ gradients. 
Table 2. Main parameters used in the PDR models of the Orion Bar.

\begin{tabular}{|c|c|c|}
\hline Model parameter & Value & Note \\
\hline FUV illumination, $G_{0}$ & $2 \times 10^{4}$ Habing & (a) \\
\hline Total depth $A_{\mathrm{V}}$ & $10 \mathrm{mag}$ & \\
\hline Thermal pressure $P_{\mathrm{th}} / k$ & $2 \times 10^{8} \mathrm{~cm}^{-3} \mathrm{~K}$ & \\
\hline Density $n_{\mathrm{H}}=n(\mathrm{H})+2 n\left(\mathrm{H}_{2}\right)$ & $n_{\mathrm{H}}=P_{\mathrm{th}} / k T_{\mathrm{k}}$ & Varying \\
\hline Cosmic Ray $\zeta_{\mathrm{CR}}$ & $10^{-16} \mathrm{H}_{2} \mathrm{~s}^{-1}$ & (b) \\
\hline$R_{\mathrm{V}}=A_{\mathrm{V}} / E_{\mathrm{B}-\mathrm{V}}$ & 5.5 & Orion $^{c}$ \\
\hline$M_{\text {gas }} / M_{\text {dust }}$ & 100 & Local ISM \\
\hline Abundance $\mathrm{O} / \mathrm{H}$ & $3.2 \times 10^{-4}$ & \\
\hline Abundance $\mathrm{C} / \mathrm{H}$ & $1.4 \times 10^{-4}$ & Orion $^{d}$ \\
\hline Abundance S / H & $1.4 \times 10^{-5}$ & Solar ${ }^{e}$ \\
\hline
\end{tabular}

Notes. ${ }^{a}$ Marconi et al. (1998). ${ }^{b}$ Indriolo et al. (2015). ${ }^{c}$ Cardelli et al. (1989). ${ }^{d}$ Sofia et al. (2004). ${ }^{e}$ Asplund et al. (2009).

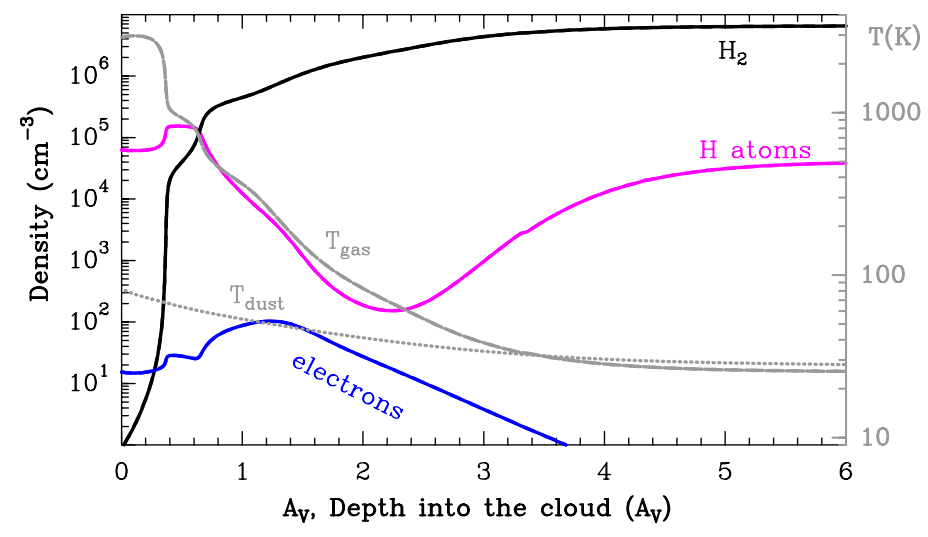

Fig. 10. Structure of an isobaric PDR representing the most FUVirradiated gas layers of the Orion Bar (see Table 2 for the adopted parameters). This plot shows the $\mathrm{H}_{2}, \mathrm{H}$, and electron density profiles (left axis scale), and the gas and dust temperatures (right axis scale).

Our chemical network is that of the Meudon code updated with the new reaction rates listed in Table 1 . This network includes updated photoreaction rates from Heays et al. (2017). To increase the accuracy of our abundance predictions, we included the explicit integration of wavelength-dependent $\mathrm{SH}, \mathrm{SH}^{+}$, and $\mathrm{H}_{2} \mathrm{~S}$ photodissociation cross sections $\left(\sigma_{\text {diss }}\right)$, as well as $\mathrm{SH}$ and $\mathrm{H}_{2} \mathrm{~S}$ photoionization cross sections $\left(\sigma_{\text {ion }}\right)$. These cross sections are shown in Fig C.1 of the Appendix. The integration is performed over the specific FUV radiation field at each position of the PDR. In particular, we took $\sigma_{\text {ion }}(\mathrm{SH})$ from Hrodmarsson et al. (2019) and $\sigma_{\text {diss }}\left(\mathrm{H}_{2} \mathrm{~S}\right)$ from Zhou et al. (2020), both determined in laboratory experiments. Figure 11 summarizes the relevant chemical network that leads to the formation of S-bearing hydrides and that we discuss in the following sections.

\subsection{Pure gas-phase PDR model results}

Figure 12 shows results of the "new gas-phase" model using the reaction rates in Table 1 . The continuous curves display the predicted fractional abundance profiles as a function of cloud depth in magnitudes of visual extinction $\left(A_{V}\right)$. The dashed curves are for a model that uses the standard thermal rates previously adopted in the literature (see, e.g., Neufeld et al. 2015). As noted by Zanchet et al. (2013a, 2019), the inclusion of $\mathrm{H}_{2}(v \geq 2)$ state-

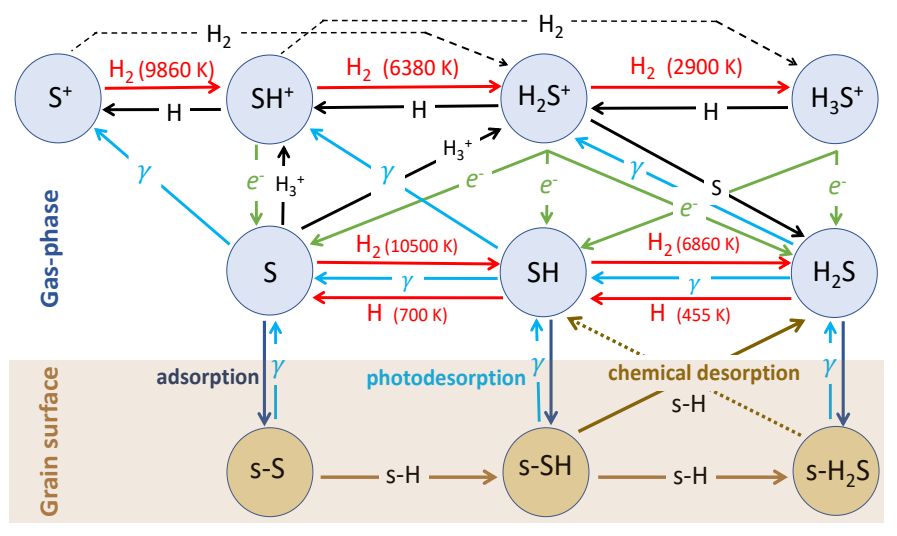

Fig. 11. Main gas and grain reactions leading to the formation of sulfur hydrides. Red arrows represent endoergic reactions (endothermicity given in units of $\mathrm{K}$ ). Dashed arrows are uncertain radiative associations (see Sect. A.3), $\gamma$ stands for a FUV photon, and "s-" for solid.

dependent quantum rates for reaction (1) enhances the formation of $\mathrm{SH}^{+}$in a narrow layer at the edge of the PDR $\left(A_{V} \simeq 0\right.$ to $\left.2 \mathrm{mag}\right)$. This agrees with the morphology of the $\mathrm{SH}^{+}$emission revealed by ALMA images (Fig. 3). For $\mathrm{H}_{2}(v=2)$, the reaction rate enhancement with respect to the thermal rate $\Delta k=k_{2}(T) / k_{0}(T)$ (see discussion by Agúndez et al. 2010) is about $4 \times 10^{8}$ at $T_{\mathrm{k}}=500 \mathrm{~K}$ (Millar et al. 1986). Indeed, when the fractional abundance of $\mathrm{H}_{2}(v=2)$ with respect to $\mathrm{H}_{2}(v=0)$, defined as $f_{2}=n\left(\mathrm{H}_{2} v=2\right) / n\left(\mathrm{H}_{2} v=0\right)$, exceeds a few times $10^{-9}$, meaning $\Delta k \cdot f_{2}>1$, reaction (1) with $\mathrm{H}_{2}(v \geq 2)$ dominates $\mathrm{SH}^{+}$formation. This reaction enhancement takes place only at the edge of the PDR, where FUV-pumped $\mathrm{H}_{2}(v \geq 2)$ molecules are abundant enough (gray dashed curves in Fig. 12) and drive the formation of $\mathrm{SH}^{+}$. The resulting $\mathrm{SH}^{+}$column density increases by an order of magnitude compared to models that use the thermal rate.

In this isobaric model, the $\mathrm{SH}^{+}$abundance peak occurs at $A_{V} \simeq 0.7 \mathrm{mag}$, where the gas density has increased from $n_{\mathrm{H}} \simeq 6 \times 10^{4} \mathrm{~cm}^{-3}$ at the PDR edge (the IF) to $\sim 5 \times 10^{5} \mathrm{~cm}^{-3}$ (at the $\mathrm{DF})$. At this point, $\mathrm{SH}^{+}$destruction is dominated by recombination with electrons and by reactive collisions with $\mathrm{H}$ atoms. This implies $D\left(\mathrm{SH}^{+}\right)\left[\mathrm{s}^{-1}\right] \sim n_{e} k_{e} \simeq n_{\mathrm{H}} k_{\mathrm{H}} \gg n\left(\mathrm{H}_{2} v \geq 2\right) k_{2}$, as we assumed in the single-slab $\mathrm{SH}^{+}$excitation models (Sec. 4.1). Therefore, only a small fraction of $\mathrm{SH}^{+}$molecules further react with $\mathrm{H}_{2}(v \geq 2)$ to form $\mathrm{H}_{2} \mathrm{~S}^{+}$. The resulting low $\mathrm{H}_{2} \mathrm{~S}^{+}$abundances limit the formation of abundant $\mathrm{SH}$ from dissociative recombinations of $\mathrm{H}_{2} \mathrm{~S}^{+}$(recall that we estimated that reaction $\mathrm{S}+\mathrm{H}_{2}(v \geq 2) \rightarrow \mathrm{SH}+\mathrm{H}$ is very slow). The $\mathrm{SH}$ abundance peak is shifted deeper inside the cloud, at about $A_{V} \simeq 1.8 \mathrm{mag}$, where $\mathrm{SH}$ forms by dissociative recombination of $\mathrm{H}_{2} \mathrm{~S}^{+}$and it is destroyed by FUV photons and reactions with $\mathrm{H}$ atoms. In these gas-phase models the $\mathrm{H}_{2} \mathrm{~S}$ abundance peaks even deeper inside the PDR, at $\mathrm{A}_{V} \simeq 5 \mathrm{mag}$, where it forms by recombinations of $\mathrm{H}_{2} \mathrm{~S}^{+}$and $\mathrm{H}_{3} \mathrm{~S}^{+}$ with electrons as well as by charge exchange $\mathrm{S}+\mathrm{H}_{2} \mathrm{~S}^{+}$. However, the new rate of reaction $\mathrm{H}_{2} \mathrm{~S}^{+}+\mathrm{H}$ is higher than assumed in the past, so the new models predict lower $\mathrm{H}_{2} \mathrm{~S}^{+}$abundances at intermediate PDR depths (thus, less $\mathrm{H}_{3} \mathrm{~S}^{+}$and $\mathrm{H}_{2} \mathrm{~S}$; see Fig. 12).

The $\mathrm{SH}$ column density predicted by the new gas-phase model is below the upper limit determined from SOFIA. However, the predicted $\mathrm{H}_{2} \mathrm{~S}$ column density is much lower than the value we derive from observations (Table 3 ) and the predicted $\mathrm{H}_{2} \mathrm{~S}$ line intensities are too faint (see Sect. 6.4).

Because the cross sections of the different $\mathrm{H}_{2} \mathrm{~S}$ photodissociation channels have different wavelength dependences (Zhou et al. 2020), the $\mathrm{H}_{2} \mathrm{~S}$ and $\mathrm{SH}$ abundances between $A_{V} \approx 2$ and 6 mag 
Table 3. Column density predictions from different PDR models (up to $A_{V}=10 \mathrm{mag}$ ) and estimated values from observations (single-slab approach).

\begin{tabular}{cccccc}
\hline \hline & $\log N\left(\mathrm{~cm}^{-2}\right)$ & & & & \\
Type of PDR model & $\mathrm{SH}^{+}$ & $\mathrm{SH}$ & $\mathrm{H}_{2} \mathrm{~S}$ & $\mathrm{H}_{2} \mathrm{~S}^{+}$ & $\mathrm{H}_{3} \mathrm{~S}^{+}$ \\
\hline Standard gas-phase & $11.0^{a}-12.2^{b}$ & $11.4^{a}-12.5^{b}$ & $11.3^{a}-12.4^{b}$ & $9.9^{a}-11.1^{b}$ & $7.8^{a}-9.0^{b}$ \\
New gas-phase (Table 1) & $12.1^{a}-13.2^{b}$ & $11.4^{a}-12.5^{b}$ & $10.6^{a}-11.7^{b}$ & $9.9^{a}-11.0^{b}$ & $7.7^{a}-8.9^{b}$ \\
Gas-grain (low $\left.E_{\mathrm{b}}, \epsilon=1 \%\right)$ & $12.0^{a}-13.2^{b}$ & $13.2^{a}-14.4^{b}$ & $12.9^{a}-14.1^{b}$ & $9.6^{a}-10.7^{b}$ & $10.1^{a}-11.2^{b}$ \\
Gas-grain (high $\left.E_{\mathrm{b}}, \epsilon=1 \%\right)$ & $12.0^{a}-13.1^{b}$ & $13.6^{a}-14.8^{b}$ & $13.7^{b}-14.8^{b}$ & $9.9^{b}-11.0^{b}$ & $10.8^{b}-12.0^{b}$ \\
\hline Estimated from observations & $\sim 13.1$ & $<13.8$ & $\sim 14.4$ & - & $<10.7$ \\
\hline
\end{tabular}

Notes. ${ }^{a}$ Column densities for a face-on PDR. ${ }^{b}$ Edge-on PDR with a tilt angle $\alpha=4^{o}$, leading to the maximum expected geometrical enhancement.

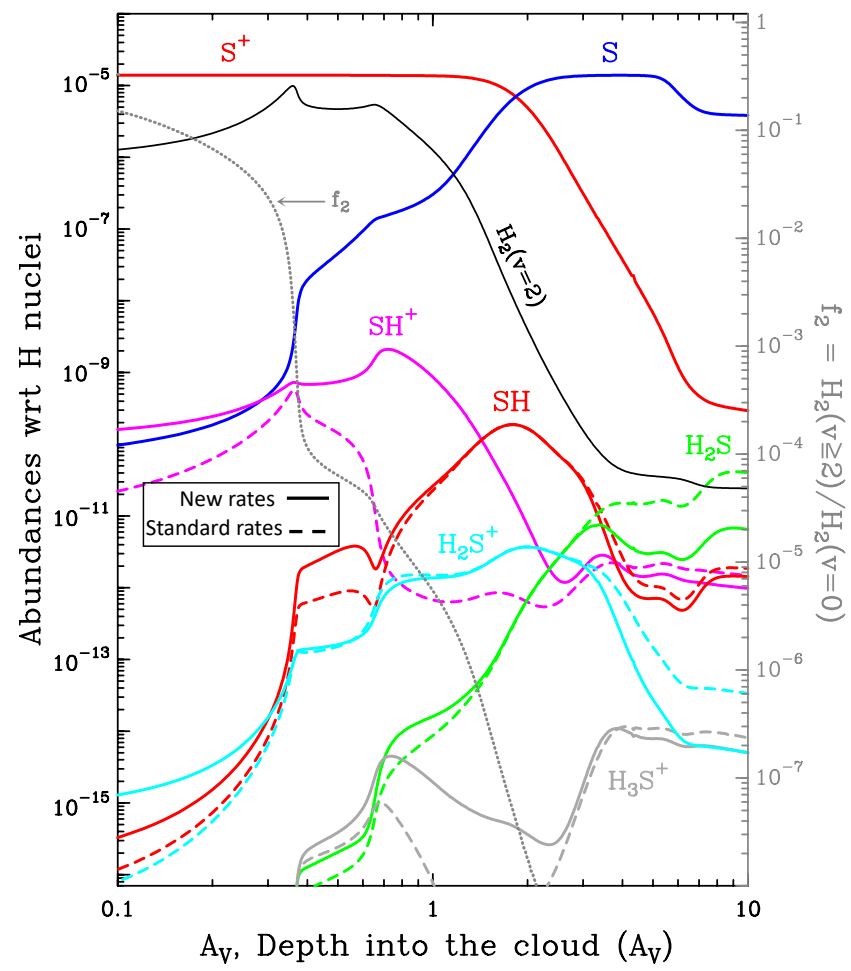

Fig. 12. Pure gas-phase PDR models of the Orion Bar. Continuous curves show fractional abundances as a function of cloud depth, in logarithm scale to better display the irradiated edge of the PDR, using the new reaction rates listed in Table 1 . The gray dotted curve shows $f_{2}$, the fraction of $\mathrm{H}_{2}$ that is in vibrationally excited levels $v \geq 2$ (right axis scale). Dashed curves are for a model using standard reaction rates.

are sensitive to the specific shape of the FUV radiation field (determined by line blanketing, dust absorption, and grain scattering; e.g., Goicoechea \& Le Bourlot 2007). Still, we checked that using steeper extinction curves does not increase $\mathrm{H}_{2} \mathrm{~S}$ column density any closer to the observed levels. This disagreement between the observationally inferred $N\left(\mathrm{H}_{2} \mathrm{~S}\right)$ column density and the predictions of gas-phase PDR models is even worse ${ }^{7}$ if one considers the uncertain rates of radiative association reactions $\mathrm{S}^{+}+\mathrm{H}_{2} \rightarrow \mathrm{H}_{2} \mathrm{~S}^{+}+h v$ and $\mathrm{SH}^{+}+\mathrm{H}_{2} \rightarrow \mathrm{H}_{3} \mathrm{~S}^{+}+h v$ included in the new gas-phase model. For the latter reaction, the main problem is that the electronic states of the reactants do not correlate with the ${ }^{1} A_{1}$ ground electronic state of the activated complex $\mathrm{H}_{3} \mathrm{~S}^{+*}$ (denoted by $*$ ). Instead, $\mathrm{H}_{3} \mathrm{~S}^{+*}$ forms in an excited triplet state $\left({ }^{3} A\right)$. Herbst et al. (1989) proposed that a spin-flip followed by a radiative association can occur in interstellar conditions

\footnotetext{
7 Older gas-phase PDR models previously predicted low $\mathrm{H}_{2} \mathrm{~S}$ column densities (Jansen et al. 1995; Sternberg \& Dalgarno 1995).
}

and form $\mathrm{H}_{3} \mathrm{~S}^{+*}\left(X^{1} A_{1}\right)$ (Millar \& Herbst 1990). In Appendix A.3, we give arguments against this mechanism. For similar reasons, Prasad \& Huntress (1982) avoided to include the $\mathrm{S}^{+}+\mathrm{H}_{2}$ radiative association in their models. Removing these reactions in pure gas-phase models drastically decreases the $\mathrm{H}_{2} \mathrm{~S}^{+}$and $\mathrm{H}_{3} \mathrm{~S}^{+}$abundances, and thus those of $\mathrm{SH}$ and $\mathrm{H}_{2} \mathrm{~S}$ (by a factor of $\sim 100$ in these models). The alternative $\mathrm{H}_{2} \mathrm{~S}^{+}$formation route through reaction $\mathrm{SH}^{+}+\mathrm{H}_{2}(v=2)$ is only efficient at the PDR surface $\left(A_{V}<1 \mathrm{mag}\right)$. This is due to the large $\mathrm{H}_{2}(v=2)$ fractional abundances, $f_{2}>10^{-6}$ at $T_{k}>500 \mathrm{~K}$, required to enhance the $\mathrm{H}_{2} \mathrm{~S}^{+}$production. Therefore, and contrary to $\mathrm{S}^{+}$destruction, reaction of $\mathrm{SH}^{+}$with $\mathrm{H}_{2}$ is not the dominant destruction pathway for $\mathrm{SH}^{+}$. Only deeper inside the $\mathrm{PDR}$, reactions of $\mathrm{S}$ with $\mathrm{H}_{3}^{+}$produce small abundances of $\mathrm{SH}^{+}$and $\mathrm{H}_{2} \mathrm{~S}^{+}$, but the hydrogenation of $\mathrm{H}_{n} \mathrm{~S}^{+}$ions is not efficient and limits the gas-phase production $\mathrm{H}_{2} \mathrm{~S}$.

\subsection{Grain surface formation of solid $\mathrm{H}_{2} \mathrm{~S}$}

Similarly to the formation of water ice $\left(\mathrm{s}-\mathrm{H}_{2} \mathrm{O}\right)$ on grains (e.g., Hollenbach et al. 2009, 2012), the formation of $\mathrm{H}_{2} \mathrm{~S}$ may be dominated by grain surface reactions followed by desorption back to the gas (e.g., Charnley 1997). Indeed, water vapor is relatively abundant in the $\operatorname{Bar}\left(N\left(\mathrm{H}_{2} \mathrm{O}\right) \approx 10^{15} \mathrm{~cm}^{-2}\right.$; Choi et al. 2014; Putaud et al. 2019) and large-scale maps show that the $\mathrm{H}_{2} \mathrm{O}$ abundance peaks close to cloud surfaces (Melnick et al. 2020).

To investigate the $\mathrm{s}-\mathrm{H}_{2} \mathrm{~S}$ formation on grains, we updated the chemical model by allowing $\mathrm{S}$ atoms to deplete onto grains as the gas temperature drops inside the molecular cloud (for the basic grain chemistry formalism, see, Hollenbach et al. 2009). The timescale of this process $\left(\tau_{\mathrm{gr}, \mathrm{S}}\right)$ goes as $x(\mathrm{~S})^{-1} n_{\mathrm{H}}^{-1} T_{\mathrm{k}}^{-1 / 2}$, where $x(\mathrm{~S})$ is the abundance of neutral sulfur atoms with respect to $\mathrm{H}$ nuclei. In a PDR, the abundance of $\mathrm{H}$ atoms is typically higher than that of $\mathrm{S}$ atoms ${ }^{8}$ and $\mathrm{H}$ atoms stick on grains more frequently than $\mathrm{S}$ atoms unless $x(\mathrm{H})<x(\mathrm{~S}) \cdot 0.18$. An adsorbed $\mathrm{H}$ atom (s-H) is weakly bound, mobile, and can diffuse throughout the grain surface until it finds an adsorbed $\mathrm{S}$ atom (s-S). If the timescale for a grain to be hit by a $\mathrm{H}$ atom $\left(\tau_{\mathrm{gr}, \mathrm{H}}\right)$ is shorter that the timescale for a s-S atom to photodesorb $\left(\tau_{\text {photdes, } S}\right)$ or sublimate $\left(\tau_{\text {subl, }}\right)$ then reaction of $\mathrm{s}-\mathrm{H}$ with $\mathrm{s}-\mathrm{S}$ will proceed and form a s-SH radical roughly upon "collision" and without energy barriers (e.g., Tielens \& Hagen 1982; Tielens 2010). Likewise, if $\tau_{\mathrm{gr}, \mathrm{H}}<\tau_{\text {photdes, SH }}$ and $\tau_{\mathrm{gr}, \mathrm{H}}<\tau_{\text {subl, SH }}$, a newly adsorbed s-H atom can diffuse, find a grain site with an s-SH radical and react without barriers to form $\mathrm{s}-\mathrm{H}_{2} \mathrm{~S}$. In these surface processes, a significant amount of $\mathrm{S}$ is ultimately transferred to $s-\mathrm{H}_{2} \mathrm{~S}$

\footnotetext{
8 We only consider the depletion of neutral $\mathrm{S}$ atoms. $\mathrm{S}^{+}$ions are expected to be more abundant than $\mathrm{S}$ atoms at the edge of the Orion Bar ( $A_{V} \lesssim 2 \mathrm{mag}$ ) where $T_{\mathrm{k}}$ and $T_{\mathrm{d}}$ are too high, and the FUV radiation field too strong, to allow the formation of abundant grain mantles.
} 

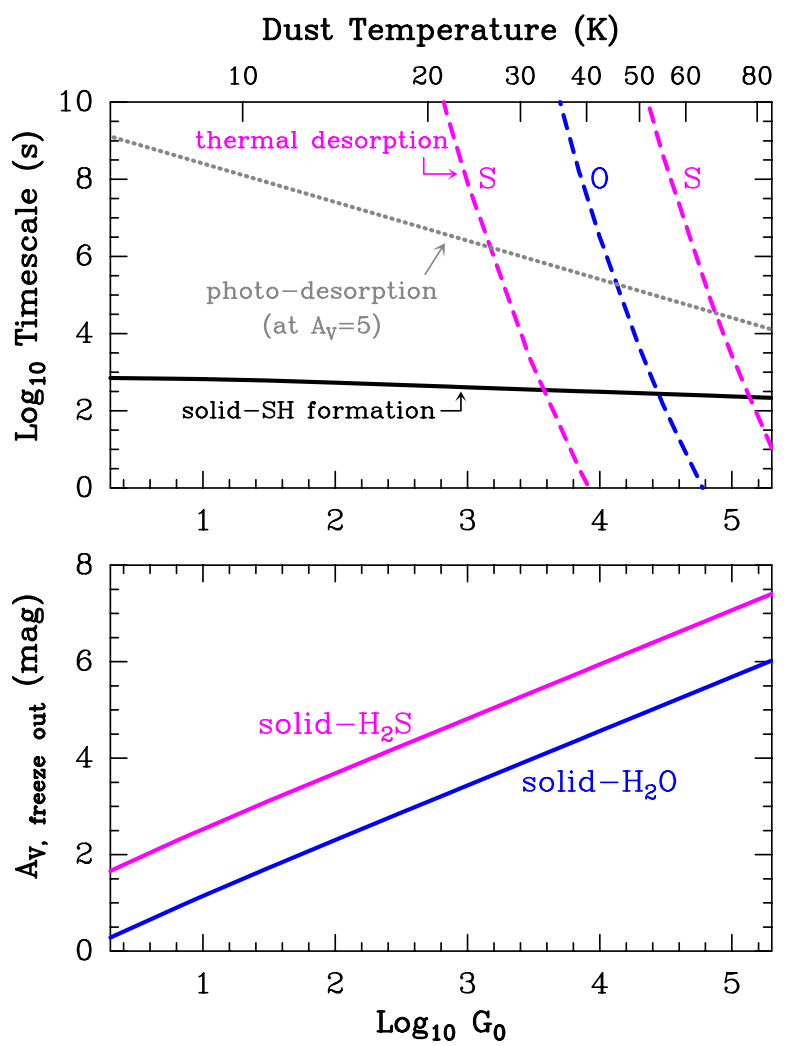

Fig. 13. Representative timescales relevant to the formation of $s-\mathrm{H}_{2} \mathrm{~S}$ and $\mathrm{s}-\mathrm{H}_{2} \mathrm{O}$ as well as their freeze-out depths. Upper panel: The continuous black curve is the timescale for a grain to be hit by an $\mathrm{H}$ atom. Once in the grain surface, the $\mathrm{H}$ atom diffuses and can react with an adsorbed $\mathrm{S}$ atom to form s-SH. The dashed magenta curves show the timescale for thermal desorption of an s-S atom $\left(E_{\mathrm{b}} / k(\mathrm{~S})=1100 \mathrm{~K}\right.$ left curve, and $2600 \mathrm{~K}$ right curve) and of an $\mathrm{s}-\mathrm{O}$ atom (blue curve; $E_{\mathrm{b}} / k(\mathrm{O})=1800 \mathrm{~K}$ ). The gray dotted curve is the photodesorption timescale of s-S. At $G_{0}$ values where the continuous line is below the dashed and dotted lines, $\mathrm{s}-\mathrm{O}$ and s-S atoms remain on grain surfaces sufficiently long to combine with an adsorbed $\mathrm{H}$ atom and form s-OH and s-SH (and then $\mathrm{s}-\mathrm{H}_{2} \mathrm{O}$ and $\mathrm{s}-\mathrm{H}_{2} \mathrm{~S}$ ). These timescales are for $n_{\mathrm{H}}=10^{5} \mathrm{~cm}^{-3}$ and $n(\mathrm{H})=100 \mathrm{~cm}^{-3}$. Bottom panel: Freeze-out depth at which most $\mathrm{O}$ and $\mathrm{S}$ are incorporated as $\mathrm{s}-\mathrm{H}_{2} \mathrm{O}$ and $\mathrm{s}-\mathrm{H}_{2} \mathrm{~S}$ (assuming no chemical desorption and $T_{\mathrm{k}}=T_{\mathrm{d}}$ ).

(e.g., Vidal et al. 2017), which can subsequently desorb: thermally, by FUV photons, or by cosmic rays. In addition, laboratory experiments show that the excess energy of certain exothermic surface reactions can promote the direct desorption of the product (Minissale et al. 2016). In particular, reaction s-H + s-SH directly desorbs $\mathrm{H}_{2} \mathrm{~S}$ with a maximum efficiency of $\sim 60 \%$ (as observed in experiments, Oba et al. 2018). Due to the high flux of FUV photons in PDRs, chemical desorption may not always compete with photodesorption. However, it can be a dominant process inside molecular clouds (Garrod et al. 2007; Esplugues et al. 2016; Vidal et al. 2017; Navarro-Almaida et al. 2020).

The photodesorption timescale of an ice mantle is proportional to $Y^{-1} G_{0}^{-1} \exp \left(+b A_{V}\right)$, where $Y$ is the photodesorption yield (the number of desorbed atoms or molecules per incident photon) and $b$ is a dust-related FUV field absorption factor. The timescale for mantle sublimation (thermal desorption) goes as $v_{\text {ice }}^{-1} \exp \left(+E_{\mathrm{b}} / k T_{\mathrm{d}}\right)$, where $v_{\text {ice }}$ is the characteristic vibrational frequency of the solid lattice, $T_{\mathrm{d}}$ is the dust grain temperature, and $E_{\mathrm{b}} / k$ is the adsorption binding energy of the species (in $\mathrm{K}$ ). Binding energies play a crucial role in model predictions because they determine the freezing temperatures and sublimation timescales. Table 4 lists the $E_{\mathrm{b}} / k$ and $Y$ values considered here.
Table 4. Adopted binding energies and photodesorption yields.

\begin{tabular}{ccc}
\hline \hline Species & $\begin{array}{c}E_{\mathrm{b}} / k \\
(\mathrm{~K})\end{array}$ & $\begin{array}{c}\text { Yield } \\
(\text { FUV photon })^{-1}\end{array}$ \\
\hline $\mathrm{S}$ & $1100^{a} / 2600^{b}$ & $10^{-4}$ \\
$\mathrm{SH}$ & $1500^{a} / 2700^{b}$ & $10^{-4}$ \\
$\mathrm{H}_{2} \mathrm{~S}$ & $2700^{b, c}$ & $\left.1.2 \times 10^{-3}{ }^{\text {as }} \mathrm{H}_{2} \mathrm{~S}\right)$ \\
$\mathrm{CO}$ & $1300^{d}$ & $3 \times 10^{-3} h$ \\
$\mathrm{O}$ & $1800^{e}$ & $10^{-4 h}$ \\
$\mathrm{O}_{2}$ & $1200^{d}$ & $10^{-3 h}$ \\
$\mathrm{OH}$ & $4600^{a}$ & $10^{-3} h$ \\
$\mathrm{H}_{2} \mathrm{O}$ & $4800^{f}$ & $10^{-3} h\left(\right.$ as $\left.\mathrm{H}_{2} \mathrm{O}\right)$ \\
& & $2 \times 10^{-3} h($ as OH$)$ \\
\hline
\end{tabular}

Notes. ${ }^{a}$ Hasegawa \& Herbst (1993). ${ }^{b}$ Wakelam et al. (2017). ${ }^{c}$ Collings et al. (2004). ${ }^{d}$ Minissale et al. (2016). ${ }^{e} \mathrm{He}$ et al. (2015). ${ }^{f}$ Sandford \& Allamandola (1988). ${ }^{g}$ Fuente et al. (2017) ${ }^{h}$ See, Hollenbach et al. (2009).

Representative timescales of the basic grain processes described above are summarized in the upper panel of Fig. 13. In this plot, $T_{d}$ is a characteristic dust temperature inside the PDR, $T_{d}=\left(3 \cdot 10^{4}+2 \cdot 10^{3} G_{0}^{1.2}\right)^{0.2}$, taken from Hollenbach et al. (2009). In the upper panel, the continuous black curve is the timescale for a grain to be hit by an $\mathrm{H}$ atom $\left(\tau_{\mathrm{gr}, \mathrm{H}}\right)$. The dashed magenta curves show the timescale for thermal desorption of an s-S atom $\left(\tau_{\text {subl, } S}\right)$ (left curve for $E_{\mathrm{b}} / k(\mathrm{~S})=1100 \mathrm{~K}$ and right curve for $E_{\mathrm{b}} / k(\mathrm{~S})=2600 \mathrm{~K}$ ), and the same for an s-O atom (blue curve). The gray dotted curve is the timescale for s-S atom photodesorption ( $\tau_{\text {photodes, }} \mathrm{S}$ ) at $A_{V}=5 \mathrm{mag}$. At $G_{0}$ strengths where the continuous line is below the dashed and dotted lines, an adsorbed $\mathrm{s}-\mathrm{S}$ atom remains on the grain surface sufficiently long to react with a diffusing s-H atom, form s-SH, and ultimately s- $\mathrm{H}_{2} \mathrm{~S}$.

Figure 13 shows that, if one takes $E_{\mathrm{b}} / k(\mathrm{~S})=1100 \mathrm{~K}$ (the most common value in the literature; Hasegawa \& Herbst 1993), the formation of $\mathrm{s}-\mathrm{H}_{2} \mathrm{~S}$ is possible inside clouds illuminated by modest FUV fields, when grains are sufficiently cold $\left(T_{\mathrm{d}}<22 \mathrm{~K}\right)$. However, recent calculations of $\mathrm{s}-\mathrm{S}$ atoms adsorbed on water ice surfaces suggest higher binding energies $(\sim 2600 \mathrm{~K}$; Wakelam et al. 2017). This would imply that $\mathrm{S}$ atoms freeze at higher $T_{\mathrm{d}}$ $(\lesssim 50 \mathrm{~K})$ and that $\mathrm{s}-\mathrm{H}_{2} \mathrm{~S}$ mantles form in more strongly illuminated PDRs (the observed $T_{\mathrm{d}}$ at the edge of the Bar is $\simeq 50 \mathrm{~K}$ and decreases to $\simeq 35 \mathrm{~K}$ behind the PDR; see, Arab et al. 2012).

The freeze-out depth for sulfur in a PDR, the $A_{V}$ at which most sulfur is incorporated as $\mathrm{S}$-bearing solids $\left(\mathrm{s}-\mathrm{H}_{2} \mathrm{~S}\right.$ in our simple model) can be estimated by equating $\tau_{\mathrm{gr}, \mathrm{S}}$ and $\tau_{\text {photdes, } \mathrm{H}_{2} \mathrm{~S}}$. This implicitly assumes the $\mathrm{H}_{2} \mathrm{~S}$ chemical desorption does not dominate in FUV-irradiated regions, which is in line with the particularly large FUV absorption cross section of s- $\mathrm{H}_{2} \mathrm{~S}$ measured in laboratory experiments (Cruz-Diaz et al. 2014). With these assumptions, the lower panel of Fig. 13 shows the predicted s- $\mathrm{H}_{2} \mathrm{~S}$ and $\mathrm{s}-\mathrm{H}_{2} \mathrm{O}$ freeze-out depths. Owing to the lower abundance and higher atomic mass of sulfur atoms (i.e., grains are hit slower by $\mathrm{S}$ atoms than by $\mathrm{O}$ atoms), the $\mathrm{H}_{2} \mathrm{~S}$ freeze-out depth appears slightly deeper than that of water ice. For the FUV-illumination conditions in the Bar, the freeze-out depth of sulfur is expected at $A_{V} \gtrsim 6 \mathrm{mag}$. This implies that photodesorption of s- $\mathrm{H}_{2} \mathrm{~S}$ can produce enhanced abundances of gaseous $\mathrm{H}_{2} \mathrm{~S}$ at $A_{V}<6$ mag.

FUV-irradiation and thermal desorption of $\mathrm{H}_{2} \mathrm{~S}$ ice mantles have been studied in the laboratory (e.g., Cruz-Diaz et al. 2014; Jiménez-Escobar \& Muñoz Caro 2011). These experiments show that pure $\mathrm{s}-\mathrm{H}_{2} \mathrm{~S}$ ices thermally desorb around $82 \mathrm{~K}$, and at higher 

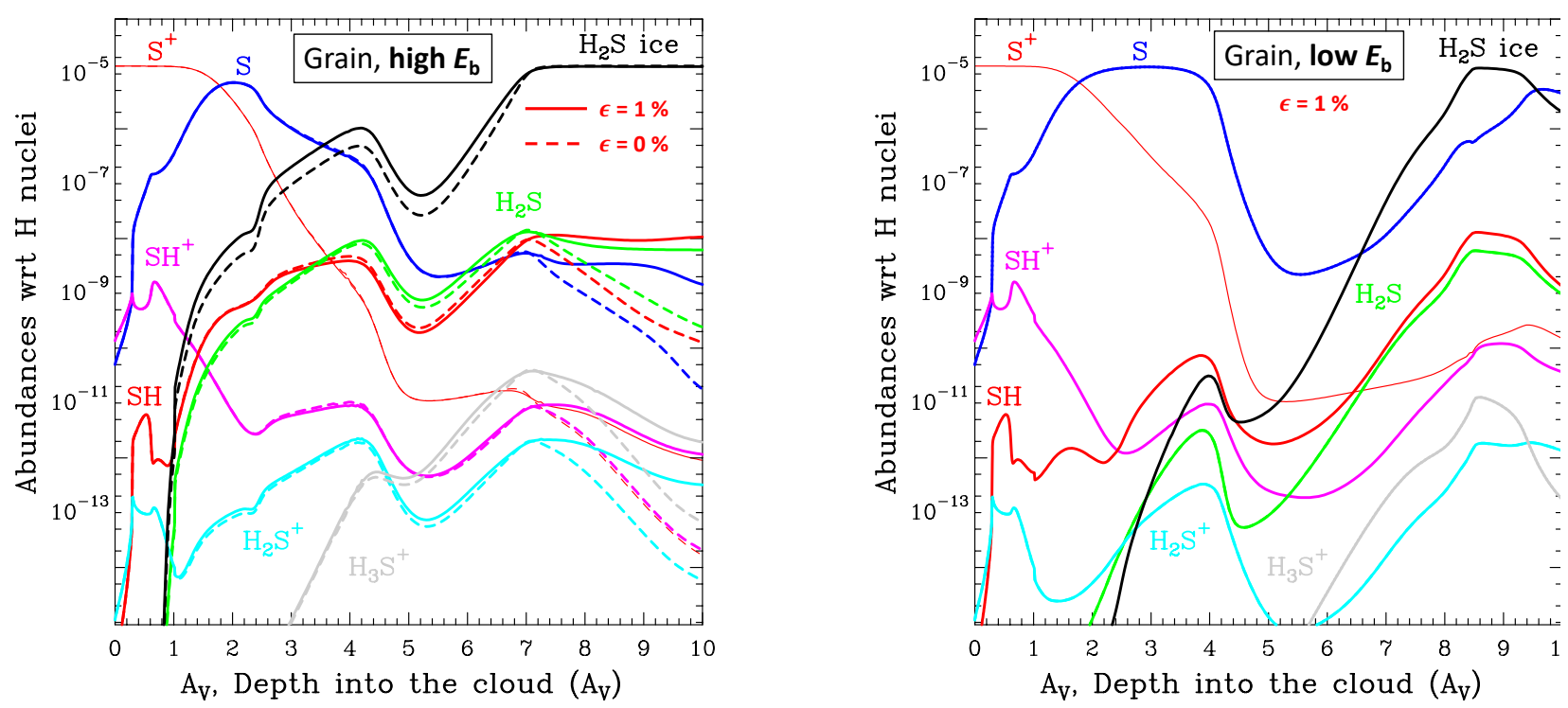

Fig. 14. Gas-grain PDR models leading to the formation of $\mathrm{s}-\mathrm{H}_{2} \mathrm{~S}$ (shown as black curves). Continuous colored curves show gas-phase fractional abundances as a function of depth into the cloud. $\epsilon$ refers to the efficiency of the chemical desorption reaction s-H $+\mathrm{s}-\mathrm{H}_{2} \mathrm{~S} \rightarrow \mathrm{SH}+\mathrm{H}_{2}(\mathrm{see}$ text). Left panel: Gas-grain high $E_{\mathrm{b}}$ model (high adsorption binding energies for $\mathrm{S}$ and $\mathrm{SH}$, see Table 4). Right panel: Low $E_{\mathrm{b}}$ model.

temperatures for $\mathrm{H}_{2} \mathrm{~S}-\mathrm{H}_{2} \mathrm{O}$ ice mixtures. These experiments determine a photodesorption yield of $Y_{\mathrm{H}_{2} \mathrm{~S}} \sim 1.2 \times 10^{-3}$ molecules per FUV photon (see also Fuente et al. 2017). Regarding surface grain chemistry, experiments show that reaction $\mathrm{s}-\mathrm{H}+\mathrm{s}-\mathrm{SH} \rightarrow \mathrm{s}-\mathrm{H}_{2} \mathrm{~S}$ is exothermic (Oba et al. 2018), whereas reaction $\mathrm{s}-\mathrm{H}+\mathrm{s}-\mathrm{H}_{2} \mathrm{~S}$, although it has an activation energy barrier of $\sim 1500 \mathrm{~K}$, it may directly desorb gaseous $\mathrm{SH}$. Finally, reaction $\mathrm{s}-\mathrm{SH}+\mathrm{s}-\mathrm{SH} \rightarrow \mathrm{s}-\mathrm{H}_{2} \mathrm{~S}_{2}$ may trigger the formation of doubly sulfuretted species, but it requires mobile s-SH radicals (e.g., Jiménez-Escobar \& Muñoz Caro 2011; Fuente et al. 2017). Here we will only consider surface reactions with mobile s-H.

\subsection{Gas-grain PDR model results}

Here we show PDR model results in which we add a simple network of gas-grain reactions for a small number of S-bearing ( $\mathrm{S}, \mathrm{SH}$, and $\left.\mathrm{H}_{2} \mathrm{~S}\right)$ and O-bearing $\left(\mathrm{O}, \mathrm{OH}, \mathrm{H}_{2} \mathrm{O}, \mathrm{O}_{2}\right.$, and $\left.\mathrm{CO}\right)$ species. These species can adsorb on grains as temperatures drop, photodesorb by FUV photons (stellar and secondary), desorb by direct impact of cosmic-rays, or sublimate at a given PDR depth (depending on $T_{\mathrm{d}}$ and on their $E_{\mathrm{b}}$ ). Grain size distributions $\left(n_{\mathrm{gr}} \propto a^{-3.5}\right.$, where $a$ is the grain radius) and gas-grain reactions are treated within the Meudon code formalism (see, Le Petit et al. 2006; Goicoechea \& Le Bourlot 2007; Le Bourlot et al. 2012; Bron et al. 2014). As grain surface chemistry reactions we include $\mathrm{s}-\mathrm{H}+\mathrm{s}-\mathrm{X} \rightarrow \mathrm{s}-\mathrm{XH}$ and $\mathrm{s}-\mathrm{H}+\mathrm{s}-\mathrm{XH} \rightarrow \mathrm{s}-\mathrm{H}_{2} \mathrm{X}$, where $\mathrm{s}-\mathrm{X}$ refers to $\mathrm{s}-\mathrm{S}$ and s-O. In addition, we add the direct chemical desorption reaction s- $\mathrm{H}+\mathrm{s}-\mathrm{SH} \rightarrow \mathrm{H}_{2} \mathrm{~S}$ with an efficiency of $50 \%$ per reactive event, and also tested different efficiencies $(\epsilon)$ for the chemical desorption process $\mathrm{s}-\mathrm{H}+\mathrm{s}-\mathrm{H}_{2} \mathrm{~S} \rightarrow \mathrm{SH}+\mathrm{H}_{2}$.

In our models we compute the relevant gas-grain timescales and atomic abundances at every depth $A_{V}$ of the PDR. If the timescale for a grain to be struck by an $\mathrm{H}$ atom $\left(\tau_{\mathrm{gr}, \mathrm{H}}\right)$ is shorter than the timescales to sublimate or to photodesorb an s-X atom or a s-XH molecule; and if $\mathrm{H}$ atoms stick on grains more frequently than $\mathrm{X}$ atoms, we simply assume these surface reactions proceed instantaneously. At large $A_{V}$, larger than the freeze-out depth, this grain chemistry builds abundant $\mathrm{s}-\mathrm{H}_{2} \mathrm{O}$ and s- $\mathrm{H}_{2} \mathrm{~S}$ ice mantles.

Figure 14 shows results of two types of gas-grain models. The only difference between them is the adopted adsorption binding energies for s-S and s-SH. Left panel is for a "high $E_{\mathrm{b}}$ " model and right panel is for a "low $E_{\mathrm{b}}$ " model (see Table 4). We note that these models do not include the gas-phase radiative association reactions $\mathrm{S}^{+}+\mathrm{H}_{2} \rightarrow \mathrm{H}_{2} \mathrm{~S}^{+}+h v$ and $\mathrm{SH}^{+}+\mathrm{H}_{2} \rightarrow \mathrm{H}_{3} \mathrm{~S}^{+}+h v$; although their effect is smaller than in pure gas-phase models.

The chemistry of the most exposed PDR surface layers $\left(A_{V} \lesssim 2 \mathrm{mag}\right.$ ) is the same to that of the gas-phase models discussed in Sect. 6.1. Photodesorption keeps dust grains free of ice mantles, and fast gas-phase ion-neutral reactions, photoreactions, and reactions with FUV-pumped $\mathrm{H}_{2}$ drive the chemistry. The resulting $\mathrm{SH}^{+}$abundance profile is nearly identical and there is no need to invoke depletion of elemental sulfur from the gas-phase to explain the observed $\mathrm{SH}^{+}$emission (see Fig. 15). Beyond these first PDR irradiated layers, the chemistry does change because the formation of $\mathrm{s}-\mathrm{H}_{2} \mathrm{~S}$ on grains and subsequent desorption alters the chemistry of the other S-bearing hydrides.

In model high $E_{\mathrm{b}}, \mathrm{S}$ atoms start to freeze out closer to the PDR edge $\left(T_{\mathrm{d}}<50 \mathrm{~K}\right)$. Because of the increasing densities and decreasing temperatures, the $\mathrm{s}-\mathrm{H}_{2} \mathrm{~S}$ abundance with respect to $\mathrm{H}$ nuclei reaches $\sim 10^{-6}$ at $A_{V} \simeq 4 \mathrm{mag}$. In model low $E_{\mathrm{b}}$, this level of s- $\mathrm{H}_{2} \mathrm{~S}$ abundance is only reached beyond an $A_{V}$ of $7 \mathrm{mag}$. At lower $A_{V}$, the formation of $\mathrm{s}-\mathrm{H}_{2} \mathrm{~S}$ on bare grains and subsequent photodesorption produces more $\mathrm{H}_{2} \mathrm{~S}$ than pure-gas phase models independently of whether $\mathrm{H}_{2} \mathrm{~S}$ chemical desorption is included or not. In these intermediate PDR layers, at $A_{V} \simeq 2-7 \mathrm{mag}$ for the strong irradiation conditions in the Bar, the flux of FUV photons drives much of the chemistry, desorbing grain mantles, preventing complete freeze out, and dissociating the gas-phase products.

There are two $\mathrm{H}_{2} \mathrm{~S}$ abundance peaks at $A_{V} \simeq 4$ and $7 \mathrm{mag}$. The $\mathrm{H}_{2} \mathrm{~S}$ abundance in these "photodesorption peaks" depends on the amount of s- $\mathrm{H}_{2} \mathrm{~S}$ mantles formed on grains and on the balance between $\mathrm{s}-\mathrm{H}_{2} \mathrm{~S}$ photodesorption and $\mathrm{H}_{2} \mathrm{~S}$ photodissociation (which now becomes the major source of $\mathrm{SH}$ ). The enhanced $\mathrm{H}_{2} \mathrm{~S}$ abundance modifies the chemistry of $\mathrm{H}_{2} \mathrm{~S}^{+}$and $\mathrm{H}_{3} \mathrm{~S}^{+}$as well: $\mathrm{H}_{2} \mathrm{~S}$ photoionization (with a threshold at $\sim 10.4 \mathrm{eV}$ ) becomes the dominant source of $\mathrm{H}_{2} \mathrm{~S}^{+}$at $A_{V} \simeq 4 \mathrm{mag}$ because the $\mathrm{H}_{2}(v \geq 2)$ abundance is too low to make reaction (2) competitive. Besides, reactions of $\mathrm{H}_{2} \mathrm{~S}$ with abundant molecular ions such as $\mathrm{HCO}^{+}$, $\mathrm{H}_{3}^{+}$, and $\mathrm{H}_{3} \mathrm{O}^{+}$dominate the $\mathrm{H}_{3} \mathrm{~S}^{+}$production. 

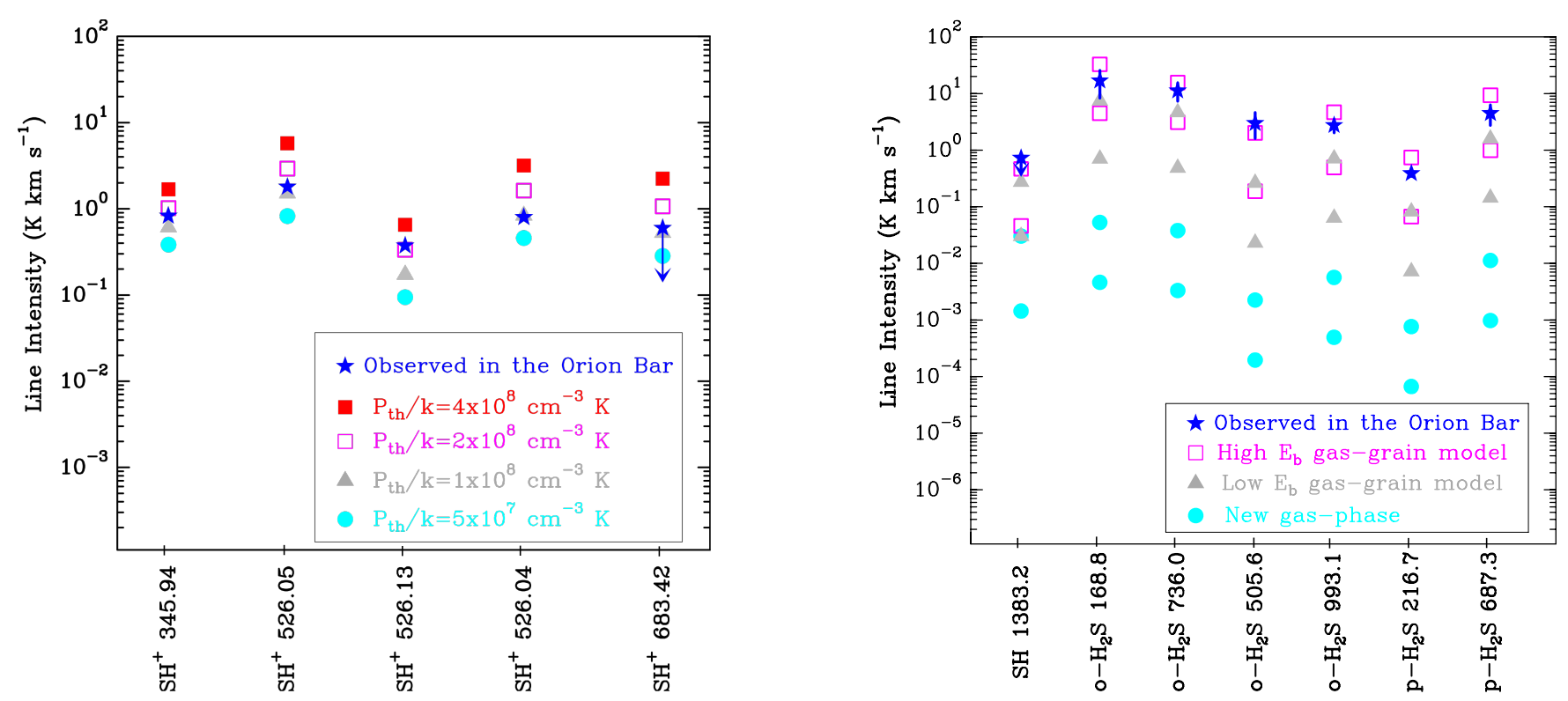

Fig. 15. Line intensity predictions for different isobaric PDR models. Calculations were carried out in a multi-slab Monte Carlo code (Sect. 4) that uses the output of the PDR model. Blue stars show the line intensities observed toward the Bar (corrected by beam dilution). Left panel: $\mathrm{SH}^{+}$ emission models for PDRs of different $P_{\mathrm{th}}$ values and $\alpha=5^{\circ}$. Right panel: $\mathrm{SH}$ and $\mathrm{H}_{2} \mathrm{~S}$ (adopting an OTP ratio of 3) emission from: high $E_{\mathrm{b}}$ (magenta squares), low $E_{\mathrm{b}}$ (gray triangles), and gas-phase (cyan circles) PDR models, all with $P_{\mathrm{th}} / k=2 \times 10^{8} \mathrm{~K} \mathrm{~cm}^{-3}$. Upper limit intensity predictions are for a PDR with an inclination angle of $\alpha=5^{\circ}$ with respect to a edge-on geometry. Lower limit intensities refer to a face-on PDR model.

Our gas-grain models predict that other S-bearing molecules, such as $\mathrm{SO}_{2}$ and $\mathrm{SO}$, can be the major sulfur reservoirs at these intermediate PDR depths. However, their abundances strongly depend on those of $\mathrm{O}_{2}$ and $\mathrm{OH}$ through reactions $\mathrm{S}+\mathrm{O}_{2} \rightarrow \mathrm{SO}+\mathrm{O}$ and $\mathrm{SO}+\mathrm{OH} \rightarrow \mathrm{SO}_{2}+\mathrm{H}$ (see e.g., Sternberg \& Dalgarno 1995; Fuente et al. 2016, 2019). These reactions link the chemistry of Sand O-bearing neutral molecules (Prasad \& Huntress 1982) and are an important sink of $\mathrm{S}$ atoms at $A_{V} \gtrsim 5 \mathrm{mag}$. However, while large column densities of $\mathrm{OH}$ have been detected in the Orion Bar ( $\approx 10^{15} \mathrm{~cm}^{-2}$; Goicoechea et al. 2011), $\mathrm{O}_{2}$ remains undetected despite deep searches (Melnick et al. 2012). Furthermore, the inferred upper limit $N\left(\mathrm{O}_{2}\right)$ columns are below the expectations of PDR models (Hollenbach et al. 2009). This discrepancy likely implies that these gas-grain models miss details of the grain surface chemistry leading to $\mathrm{O}_{2}$ (for other environments and modeling approaches see, e.g., Ioppolo et al. 2008; Taquet et al. 2016). Here we will not discuss $\mathrm{SO}_{2}, \mathrm{SO}$, or $\mathrm{O}_{2}$ further.

At large cloud depths, $A_{V} \gtrsim 8 \mathrm{mag}$, the FUV flux is largely attenuated, temperatures drop, the chemistry becomes slower, and other chemical processes dominate. The $\mathrm{H}_{2} \mathrm{~S}$ abundance is controlled by the chemical desorption reaction s- $\mathrm{H}+\mathrm{s}-\mathrm{SH} \rightarrow \mathrm{H}_{2} \mathrm{~S}$. This process keeps a floor of detectable $\mathrm{H}_{2} \mathrm{~S}$ abundances $\left(>10^{-9}\right)$ in regions shielded from stellar FUV radiation. In addition, and although not energetically favorable, the chemical desorption $\mathrm{s}-\mathrm{H}+\mathrm{s}-\mathrm{H}_{2} \mathrm{~S} \rightarrow \mathrm{SH}+\mathrm{H}_{2}$ enhances the $\mathrm{SH}$ production at large $A_{V}$ (the enhancement depends on the desorption efficiency $\epsilon$ ), which in turn boosts the abundances of other S-bearing species, including that of neutral $\mathrm{S}$ atoms.

The $\mathrm{H}_{2} \mathrm{~S}$ abundances predicted by the high $E_{\mathrm{b}}$ model reproduce the $\mathrm{H}_{2} \mathrm{~S}$ line intensities observed in the Bar (Sect. 6.4). In this model s- $\mathrm{H}_{2} \mathrm{~S}$ becomes the main sulfur reservoir. However, we stress that here we do not consider the formation of more complex S-bearing ices such as s-OCS, s- $\mathrm{H}_{2} \mathrm{~S}_{2}, \mathrm{~s}-\mathrm{S}_{n}, \mathrm{~s}-\mathrm{SO}_{2}$ or s-HSO (Jiménez-Escobar \& Muñoz Caro 2011; Vidal et al. 2017; Laas \& Caselli 2019). Together with our steady-state solution of the chemistry, this implies that our predictions are not precise deep inside the PDR. However, we recall that our observations refer to the edge of the Bar, so it is not plausible that the model conditions at $A_{V} \gtrsim 8 \mathrm{mag}$ represent the line of sight we observe.

Model low $E_{\mathrm{b}}$ produces less $\mathrm{H}_{2} \mathrm{~S}$ in the PDR layers below $A_{V} \lesssim 8$ mag because $\mathrm{S}$ atoms do not freeze until the dust temperature drops deep inside the PDR. Even beyond these layers, thermal desorption of s-S maintains higher abundances of $\mathrm{S}$ atoms at large depths. Indeed, model low $E_{\mathrm{b}}$ predicts that the major sulfur reservoir deep inside the cloud are gas-phase $S$ atoms. This agrees with recent chemical models of cold dark clouds (Vidal et al. 2017; Navarro-Almaida et al. 2020).

\subsection{Line intensity comparison and $\mathrm{H}_{2} \mathrm{~S}$ ortho-to-para ratio}

We now specifically compare the $\mathrm{SH}^{+}$, $\mathrm{SH}$, and $\mathrm{H}_{2} \mathrm{~S}$ line intensities implied by the different PDR models with the intensities observed toward the DF position of the Bar. We used the output of the PDR models $-T_{\mathrm{k}}, T_{\mathrm{d}}, n\left(\mathrm{H}_{2}\right), n(\mathrm{H}), n_{e}, n\left(\mathrm{SH}^{+}\right), n(\mathrm{SH})$, and $n\left(\mathrm{H}_{2} \mathrm{~S}\right)$ profiles from $A_{V}=0$ to $10 \mathrm{mag}$ - as input for a multi-slab Monte Carlo model of their line excitation, including formation pumping (formalism presented in Sect. 4) and radiative transfer. As the Orion Bar is not a perfectly edge-on, this comparison requires a knowledge of the tilt angle $(\alpha)$ with respect to a pure edge-on PDR. Different studies suggest $\alpha$ of $\approx 5^{\circ}$ (e.g., Jansen et al. 1995; Melnick et al. 2012; Andree-Labsch et al. 2017). This inclination implies an increase in line-of-sight column density, compared to a face-on PDR, by a geometrical factor $(\sin \alpha)^{-1}$. It also means that optically thin lines are limb-brightened.

The left panel of Fig. 15 shows $\mathrm{SH}^{+}$line intensity predictions for isobaric PDR models of different $P_{\text {th }}$ values (leading to different $T_{\mathrm{k}}$ and $n_{\mathrm{H}}$ profiles). Since the bulk of the $\mathrm{SH}^{+}$emission arises from the PDR edge ( $A_{V} \simeq 0$ to 2 mag) all models (gas-phase or gas-grain) give similar results. The best fit is for $P_{\text {th }} \simeq(1-2) \times 10^{8} \mathrm{~cm}^{-3} \mathrm{~K}$ and $\alpha \simeq 5^{\circ}$. These high pressures, at least close to the DF, agree with those inferred from ALMA images of $\mathrm{HCO}^{+}(J=4-3)$ emission (Goicoechea et al. 2016), Herschel observations of high- $J$ CO lines (Joblin et al. 2018), and IRAM $30 \mathrm{~m}$ detections of carbon recombination lines (Cuadrado et al. 2019). 

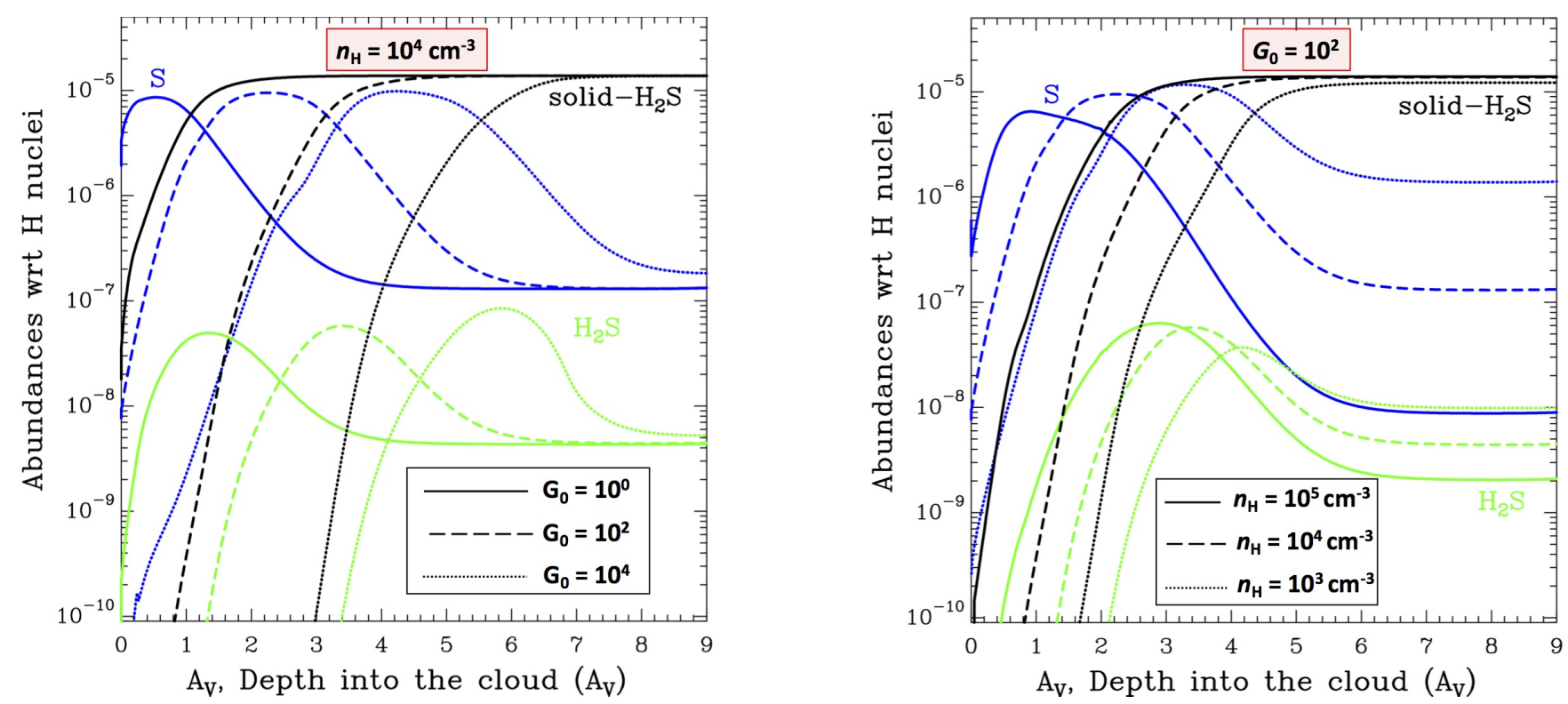

Fig. 16. Constant density gas-grain PDR models using the high $E_{\mathrm{b}}$ chemical network and undepleted sulfur elemental abundances. Left panel: Effects of changing the FUV radiation field. Right panel: Effects of varying the gas density.

Right panel of Fig. 15 shows $\mathrm{SH}$ and $\mathrm{H}_{2} \mathrm{~S}$ line emission predictions for the high $E_{\mathrm{b}}$ gas-grain model (magenta squares), low $E_{\mathrm{b}}$ gas-grain model (gray triangles), and a pure gas-phase model (cyan circles). For each model, the upper limit intensities refer to radiative transfer calculations with an inclination angle $\alpha=5^{\circ}$. The lower intensity limits refer to a face-on PDR. Gasphase models largely underestimate the observed $\mathrm{H}_{2} \mathrm{~S}$ intensities. Model low $E_{\mathrm{b}}$ produces higher $\mathrm{H}_{2} \mathrm{~S}$ columns and brighter $\mathrm{H}_{2} \mathrm{~S}$ lines, but still below the observed levels (by up to a factor of ten). Model high $E_{\mathrm{b}}$ provides a good agreement with observations; the two possible inclinations bracket the observed intensities, and it should be considered as the reference model of the Bar. It is also consistent with the observational SH upper limits.

Our observations and models provide a (line-of-sight) $N\left(o-\mathrm{H}_{2} \mathrm{~S}\right) / N\left(p-\mathrm{H}_{2} \mathrm{~S}\right)$ OTP ratio of $2.9 \pm 0.3$, consistent with the (gas-phase) high-temperature statistical equilibrium value. However, the cold "nuclear-spin-temperatures" $\left(T_{\text {spin }} \ll T_{\mathrm{k}}\right.$; see definition in eq. D.1) implied by the low water vapor OTP ratios observed in some sources $(<2.5)$ have been associated with the temperature of the ice mantles where $\mathrm{H}_{2} \mathrm{O}$ molecules might have formed (i.e., $T_{\mathrm{spin}} \simeq T_{\mathrm{d}}$; Mumma et al. 1987; Lis et al. 2013). In the case of $\mathrm{H}_{2} \mathrm{~S}$, our derived OTP ratio toward the DF position implies any $T_{\text {spin }}$ above $30 \pm 10 \mathrm{~K}$ (see Fig. D.1). Hence, this temperature might be also compatible with $\mathrm{s}-\mathrm{H}_{2} \mathrm{~S}$ formation ${ }^{9}$ in warm grains if $T_{\text {spin }} \simeq T_{\mathrm{d}}$ upon formation is preserved in the gas-phase after photodesorption (e.g., Guzmán et al. 2013). Interestingly, the $\mathrm{H}_{2} \mathrm{O}$ OTP ratio derived from observations of the Orion Bar is $2.8 \pm 0.1$ (Putaud et al. 2019) and implies $T_{\text {spin }}\left(\mathrm{H}_{2} \mathrm{O}\right)=35 \pm 2 \mathrm{~K}$. This value is compatible with $T_{\text {spin }}\left(\mathrm{H}_{2} \mathrm{~S}\right)$ and might reflect the similar $T_{\mathrm{d}}$ of the PDR layers where most s- $\mathrm{H}_{2} \mathrm{O}$ and s- $\mathrm{H}_{2} \mathrm{~S}$ form and photodesorb. Nevertheless, laboratory experiments have chal-

\footnotetext{
9 Crockett et al. (2014) inferred $N\left(o-\mathrm{H}_{2} \mathrm{~S}\right) / N\left(p-\mathrm{H}_{2} \mathrm{~S}\right)=2.5 \pm 0.8$ in the hot core of Orion KL using LTE rotational digrams. However, they favored an OTP ratio of $1.7 \pm 0.8$ based on the column density ratio of selected pairs of rotational levels with similar energies. This latter OTP ratio implies $T_{\text {spin }}\left(\mathrm{H}_{2} \mathrm{~S}\right) \simeq 12 \mathrm{~K}$ (Fig. D.1), perhaps related to much colder dust grains than in PDRs or to colder gas conditions just before the hot core phase; so that reactive collisions did not have time to establish the statistical equilibrium value. We note that the observed OTP ratios of $\mathrm{H}_{2} \mathrm{CO}, \mathrm{H}_{2} \mathrm{CS}$, and $\mathrm{H}_{2} \mathrm{CCO}$ in the Bar are also 3 (Cuadrado et al. 2017).
}

lenged this $T_{\mathrm{spin}} \simeq T_{\mathrm{d}}$ association, at least for $\mathrm{s}-\mathrm{H}_{2} \mathrm{O}$ : cold water ice surfaces, at $10 \mathrm{~K}$, photodesorb $\mathrm{H}_{2} \mathrm{O}$ molecules with an OTP ratio of $\sim 3$ (Hama et al. 2016). Follow up observations of $p-\mathrm{H}_{2} \mathrm{~S}$ lines across the Bar will allow us to study possible variations of the OTP ratio as $G_{0}$ diminishes and grains get colder.

\subsection{Generalization to different $G_{0}$ and $n_{\mathrm{H}}$ conditions}

In this section we generalize our results to a broader range of gas densities and FUV illumination conditions (i.e., to clouds with different $G_{0} / n_{\mathrm{H}}$ ratios). We run several PDR models using the high $E_{\mathrm{b}}$ gas-grain chemistry. The main difference compared to the Orion Bar models is that here we model constant density clouds with standard interstellar grain properties $\left(R_{V}=3.1\right)$. Figure 16 (left panel) shows models of clouds with constant $n_{\mathrm{H}}=10^{4} \mathrm{~cm}^{-3}$ and varying FUV radiation fields, while Fig. 16 (right panel) show models of constant FUV illumination $\left(G_{0}=100\right)$ and varying gas densities ${ }^{10}$. The main result of this study is the similar gas-phase $\mathrm{H}_{2} \mathrm{~S}$ column density (a few $10^{14} \mathrm{~cm}^{-2}$ up to $A_{V}=10$ ) and $\mathrm{H}_{2} \mathrm{~S}$ abundance peak (a few $10^{-8}$ close to the FUV-irradiated cloud edge) predicted by these models nearly irrespective of $G_{0}$ and $n_{\mathrm{H}}$. A similar conclusion was reached previously for water vapor in FUV-illuminated clouds (Hollenbach et al. 2009, 2012). Increasing $G_{0}$ shifts the position of the $\mathrm{H}_{2} \mathrm{~S}$ abundance peak to larger $A_{V}$ until the rate of $\mathrm{S}$ atoms sticking on grains balances the $\mathrm{H}_{2} \mathrm{~S}$ photodissociation rate (the dominant $\mathrm{H}_{2} \mathrm{~S}$ destruction mechanism except in shielded gas; see also Fig.13). Since s- $\mathrm{H}_{2} \mathrm{~S}$ photodesorption and $\mathrm{H}_{2} \mathrm{~S}$ photodissociation rates depend on $G_{0}$, the peak $\mathrm{H}_{2} \mathrm{~S}$ abundance in the PDR is roughly the same independently of $G_{0}$. On the other hand, the formation rate of $\mathrm{s}-\mathrm{H}_{2} \mathrm{~S}$ mantles depends on the product $n(\mathrm{~S}) n_{\mathrm{gr}} \propto n_{\mathrm{H}}^{2}$, whereas the $\mathrm{H}_{2} \mathrm{~S}$ photodesorption rate depends on $n_{\mathrm{gr}} \propto n_{\mathrm{H}}$. Hence, the $\mathrm{H}_{2} \mathrm{~S}$ abundance peak moves toward the cloud surface for denser PDRs (like the Orion Bar). The exact abundance value depends on the adopted grain-size distribution and on the $\mathrm{H}_{2} \mathrm{~S}$ photodesorption yield (which is well constrained by experiments; see, Cruz-Diaz et al. 2014; Fuente et al. 2017).

${ }_{10}$ In these models we consider undepleted $[\mathrm{S} / \mathrm{H}]$ abundances and only the chemical desorption $\mathrm{s}-\mathrm{H}+\mathrm{s}-\mathrm{SH} \rightarrow \mathrm{H}_{2} \mathrm{~S}$ (with a $50 \%$ efficiency). 
The role of chemical desorption increases and can dominate beyond the photodesorption peak as the flux of stellar FUV photons is attenuated. Here we do not carry out an exhaustive study of this mechanism, which is hard to model in full detail because its efficiency decreases considerably with the properties of grain surfaces (bare vs. icy; see e.g., Minissale \& Dulieu 2014). In our models, and depending on $\zeta_{\mathrm{CR}}$, photodesorption by secondary FUV photons can also be important in cloud interiors. These processes limit the conversion of most of the sulfur reservoir into $\mathrm{S}$-bearing ices and increase the abundance of other gas-phase species deep inside clouds, notably $\mathrm{S}$ atoms and $\mathrm{H}_{2} \mathrm{~S}$ molecules.

The $\mathrm{H}_{2} \mathrm{~S}$ abundance in shielded gas depends on the destruction rate by gas-phase reactions different than photodissociation, in particular $\mathrm{H}_{2} \mathrm{~S}$ reactions with $\mathrm{H}_{3}^{+}$. The $\mathrm{H}_{3}^{+}$abundance increases with $\zeta_{\mathrm{CR}}$ and decreases with the electron density. Figure 16 (right) shows models of constant $G_{0}$ and constant $\zeta_{\mathrm{CR}}$ in which the $\mathrm{H}_{2} \mathrm{~S}$ abundance at large depths increases with decreasing density (more penetration of FUV photons, more ionization, more electrons, less $\mathrm{H}_{3}^{+}$). The lowest gas density model, $n_{\mathrm{H}}=10^{3} \mathrm{~cm}^{-3}$, shows the highest $\mathrm{H}_{2} \mathrm{~S}$ abundance at large $A_{V}$. Because $\mathrm{S}$ freeze-out is less efficient at low densities, the lowdensity model shows higher gas-phase $\mathrm{S}$ abundances at large depths, making atomic $\mathrm{S}$ a dominant gas-phase sulfur reservoir. Unfortunately, direct observation of atomic $\mathrm{S}$ in cold gas is complicated, which makes it difficult to benchmark this prediction.

In warm PDRs, in addition to $S$ radio recombination lines (e.g., Smirnov et al. 1995), the ${ }^{3} P$ fine-structure lines of atomic sulfur, the $\left[\mathrm{S}_{\mathrm{I}}\right] 25,56 \mu \mathrm{m}$ lines, can be interesting diagnostics of gas physical conditions and of $[\mathrm{S} / \mathrm{H}]$ abundances. Unfortunately, the low sensitivity of previous infrared telescopes was not sufficient to detect the $\left[\mathrm{S}_{\mathrm{I}}\right] 25 \mu \mathrm{m}$ line $\left(\Delta E_{12}=570 \mathrm{~K}\right)$ in the Orion Bar (Rosenthal et al. 2000); although it is detected in protostellar outflows (e.g., Neufeld et al. 2009; Goicoechea et al. 2012). Moreover, the ${ }^{3} P_{2-}{ }^{1} D_{2}$ forbidden line of atomic sulfur at $1.082 \mu \mathrm{m}$ can be an interesting tracer of the ionization and dissociation fronts in PDRs. Some of these lines will be accesible to high-angularresolution and high sensitivity observations with JWST.

\subsubsection{The origin of $\mathrm{H}_{2} \mathrm{~S}$ emission in other environments}

Irrespective of $n_{\mathrm{H}}$ and $G_{0}$, grain surface formation of s- $\mathrm{H}_{2} \mathrm{~S}$ and photodesorption back to the gas-phase lead to $\mathrm{H}_{2} \mathrm{~S}$ column densities of a few $10^{14} \mathrm{~cm}^{-2}$ in PDRs. This is in agrement with the observed column in the $\operatorname{Bar}\left(G_{0} \approx 10^{4}\right)$ as well as at the mildly illuminated rims of TMC- 1 and Barnard $1 \mathrm{~b}$ clouds $\left(G_{0} \approx 10\right.$; NavarroAlmaida et al. 2020). The inferred $\mathrm{H}_{2} \mathrm{~S}$ abundance in the shielded interior of these dark clouds $\left(A_{V}>10 \mathrm{mag}\right)$ drops to a few $10^{-9}$, but the species clearly does not disappear from the gas $\left(\mathrm{N}_{(\mathrm{H}} \mathrm{S}\right)$ of a few $10^{13} \mathrm{~cm}^{-2}$; Navarro-Almaida et al. 2020). Interestingly, neither in the Bar the $\mathrm{H}_{2} \mathrm{~S}$ line emission at $\sim 168 \mathrm{GHz}$ decreases much behind the PDR (Fig. 1) even if the flux of FUV photons is largely attenuated compared to the irradiated PDR edge.

Despite oxygen is $\sim 25$ times more abundant than sulfur, the $\mathrm{H}_{2} \mathrm{O}$ to $\mathrm{H}_{2} \mathrm{~S}$ column density ratio in the Orion Bar PDR is only about $\sim 5$. This similarity must also reflect the higher abundances of $\mathrm{CO}$ compared to $\mathrm{CS}$. Furthermore, the $\mathrm{H}_{2} \mathrm{~S}$ column density in cold cores is strikingly similar to that of water vapor (Caselli et al. 2010, 2012). This coincidence points to a more efficient desorption mechanism of $\mathrm{s}-\mathrm{H}_{2} \mathrm{~S}$ compared to $\mathrm{s}-\mathrm{H}_{2} \mathrm{O}$ in gas shielded from stellar FUV photons. Navarro-Almaida et al. (2020) argues that chemical desorption is able to reproduce the observed $\mathrm{H}_{2} \mathrm{~S}$ abundance floor if the efficiency of this process diminishes as ice grain mantles get thicker inside cold dense cores.
Turning back to warmer star-forming environments, our predicted $\mathrm{H}_{2} \mathrm{~S}$ abundance in FUV-illuminated gas is comparable to that observed toward many hot cores $\left(\sim 10^{-9}-10^{-8}\right.$; van der Tak et al. 2003; Herpin et al. 2009). In these massive protostellar environments, thermal desorption of icy mantles, suddenly heated to $T_{\mathrm{d}} \gtrsim 100 \mathrm{~K}$ by the luminosity of the embedded massive protostar, drives the $\mathrm{H}_{2} \mathrm{~S}$ production. Early in their evolution, young hot cores $\left(\lesssim 10^{4} \mathrm{yr}\right)$ can show even higher abundances of recently desorbed $\mathrm{H}_{2} \mathrm{~S}$ (before further chemical processing takes place in the gas-phase; e.g., Charnley 1997; Hatchell et al. 1998; Jiménez-Serra et al. 2012; Esplugues et al. 2014). Indeed, Crockett et al. (2014) reports a gas-phase $\mathrm{H}_{2} \mathrm{~S}$ abundance of several $10^{-6}$ toward the hot core in Orion KL. This high value likely reflects the minimum $\mathrm{s}-\mathrm{H}_{2} \mathrm{~S}$ abundance locked as $\mathrm{s}-\mathrm{H}_{2} \mathrm{~S}$ mantles just before thermal desorption. In addition, the $\mathrm{H}_{2} \mathrm{~S}$ abundance in the Orion Bar is only slightly lower than that inferred in protostellar outflows (several $10^{-8}$ ). In these regions, fast shocks erode and sputter the grain mantles, releasing a large fraction of their molecular content and activating a high-temperature gas-phase chemistry that quickly reprocesses the gas (e.g., Holdship et al. 2019). All in all, it seems reasonable to conclude that everywhere s- $\mathrm{H}_{2} \mathrm{~S}$ grain mantles form, or already formed in a previous evolutionary stage, emission lines from gas-phase $\mathrm{H}_{2} \mathrm{~S}$ will be detectable.

In terms of its detectability with single-dish telescopes, $\mathrm{H}_{2} \mathrm{~S}$ rotational lines are bright in hot cores $\left(T_{\text {peak, }} 168 \mathrm{GHz} \simeq 30 \mathrm{~K}\right.$ in Orion KL but $\simeq 1-3 \mathrm{~K}$ toward most hot cores; Tercero et al. 2010; van der Tak et al. 2003; Herpin et al. 2009), in strongly irradiated PDRs ( $\simeq 6 \mathrm{~K}$, this work), and in lower-illumination PDRs such as the Horsehead ( $~ 1 \mathrm{~K}$; Rivière-Marichalar et al. 2019). The $\mathrm{H}_{2} \mathrm{~S}$ emission is fainter toward cold dark clouds $(\simeq 0.2 \mathrm{~K}$ in TMC-1; Navarro-Almaida et al. 2020) and protostellar outflows $(\simeq 0.6 \mathrm{~K}$ in L1157; Holdship et al. 2019). These line intensity differences are mostly produced by different gas physical conditions and not by enormous changes of the $\mathrm{H}_{2} \mathrm{~S}$ abundance.

Finally, $\mathrm{H}_{2} \mathrm{~S}$ is also detected outside the Milky Way (firstly by Heikkilä et al. 1999). Lacking enough spatial-resolution it is more difficult to determine the origin of the extragalactic $\mathrm{H}_{2} \mathrm{~S}$ emission. The derived abundances in starburst galaxies such as NGC 253 $\left(\sim 10^{-9}\right.$; Martín et al. 2006) might be interpreted as arising from a collection of spatially unresolved hot cores (Martín et al. 2011). However, hot cores have low filling factors at star-forming cloud scales. Our study suggests that much of this emission can arise from (the most common) extended molecular gas illuminated by stellar FUV radiation (e.g., Goicoechea et al. 2019).

\section{Summary and conclusions}

We carried out a self-consistent observational and modeling study of the chemistry of S-bearing hydrides in FUV-illuminated gas. We obtained the following results:

- ALMA images of the Orion Bar show that $\mathrm{SH}^{+}$is confined to narrow gas layers of the PDR edge, close to the $\mathrm{H}_{2}$ dissociation front. Pointed observations carried out with the IRAM $30 \mathrm{~m}$ telescope show bright $\mathrm{H}_{2}^{32} \mathrm{~S}, \mathrm{H}_{2}^{34} \mathrm{~S}, \mathrm{H}_{2}^{33} \mathrm{~S}$ emission toward the PDR (but no $\mathrm{H}_{3} \mathrm{~S}^{+}$, a key gas precursor of $\mathrm{H}_{2} \mathrm{~S}$ ) as well as behind the Bar, where the flux of FUV photons is largely attenuated. SOFIA observations provide tight limits to the SH emission.

- The $\mathrm{SH}^{+}$line emission arises from a high-pressure gas component, $P_{\text {th }} \simeq(1-2) \times 10^{8} \mathrm{~cm}^{-3} \mathrm{~K}$, where $\mathrm{SH}^{+}$ions are destroyed by reactive collisions with $\mathrm{H}$ atoms and electrons (as most $\mathrm{H}_{n} \mathrm{~S}^{+}$ ions do). We derive $N\left(\mathrm{SH}^{+}\right) \simeq 10^{13} \mathrm{~cm}^{-2}$ and an abundance peak of several $\sim 10^{-9} . \mathrm{H}_{2} \mathrm{~S}$ shows larger column densities toward the PDR, $N\left(\mathrm{H}_{2} \mathrm{~S}\right)=N\left(o-\mathrm{H}_{2} \mathrm{~S}\right)+N\left(p-\mathrm{H}_{2} \mathrm{~S}\right) \simeq 2.5 \times 10^{14} \mathrm{~cm}^{-2}$. Our tentative detection of $\mathrm{SH}$ translates into an upper limit 
column density ratio $N(\mathrm{SH}) / N\left(\mathrm{H}_{2} \mathrm{~S}\right)$ of $<0.2-0.6$, already lower than the ratio of 1.1-3.0 observed in low-density diffuse molecular clouds (Neufeld et al. 2015). This implies an enhanced $\mathrm{H}_{2} \mathrm{~S}$ production mechanism in FUV-illuminated dense gas.

- All gas-phase reactions $\mathrm{X}+\mathrm{H}_{2}(v=0) \rightarrow \mathrm{XH}+\mathrm{H}\left(\right.$ with $\mathrm{X}=\mathrm{S}^{+}$, $\mathrm{S}, \mathrm{SH}^{+}$, or $\mathrm{H}_{2} \mathrm{~S}^{+}$) are highly endoergic. While reaction of FUV-pumped $\mathrm{H}_{2}(v \geq 2)$ molecules with $\mathrm{S}^{+}$ions becomes exoergic and explains the observed levels of $\mathrm{SH}^{+}$, further reactions of $\mathrm{H}_{2}(v \geq 2)$ with $\mathrm{SH}^{+}$or with neutral $\mathrm{S}$ atoms, both reactions studied here through $a b$ initio quantum calculations, do not form enough $\mathrm{H}_{2} \mathrm{~S}^{+}$or $\mathrm{H}_{3} \mathrm{~S}^{+}$to ultimately produce abundant $\mathrm{H}_{2} \mathrm{~S}$. In particular, pure gas-phase models underestimate the $\mathrm{H}_{2} \mathrm{~S}$ column density observed in the Orion Bar by more than two orders of magnitude. This implies that these models miss the main $\mathrm{H}_{2} \mathrm{~S}$ formation route. The disagreement is even worse as we favor, after considering the potential energy surfaces of the $\mathrm{H}_{2} \mathrm{~S}^{+*}$ and $\mathrm{H}_{3} \mathrm{~S}^{+*}$ complexes, that the radiative associations $\mathrm{S}^{+}+\mathrm{H}_{2} \rightarrow \mathrm{H}_{2} \mathrm{~S}^{+}+h v$ and $\mathrm{SH}^{+}+\mathrm{H}_{2} \rightarrow \mathrm{H}_{3} \mathrm{~S}^{+}+h v$ may actually not occur or possess slower rates than considered in the literature. - To overcome these bottlenecks, we built PDR models that include a simple network of gas-grain and grain surface reactions. The higher binding energies of $\mathrm{S}$ and $\mathrm{SH}$ suggested by recent studies imply that bare grains start to grow s- $\mathrm{H}_{2} \mathrm{~S}$ mantles not far from the illuminated edges of molecular clouds. Indeed, the observed $N\left(\mathrm{H}_{2} \mathrm{~S}\right)$ in the Orion Bar can only be explained by the freeze-out of $\mathrm{S}$ atoms, grain surface formation of $\mathrm{s}-\mathrm{H}_{2} \mathrm{~S}$ mantles, and subsequent photodesorption back to the gas phase. The inferred $\mathrm{H}_{2} \mathrm{~S}$ OTP ratio of $2.9 \pm 0.3$ (equivalent to $T_{\text {spin }} \geq 30 \mathrm{~K}$ ) is compatible with the high-temperature statistical ratio as well as with warm grain surface formation if $T_{\text {spin }} \simeq T_{\mathrm{d}}$ and if $T_{\text {spin }}$ is preserved in the gas-phase after desorption.

- Comparing observations with chemical and excitation models, we conclude that the $\mathrm{SH}^{+}$-emitting layers at the edge of the Orion Bar $\left(A_{V}<2 \mathrm{mag}\right)$ are charaterized by no or very little depletion of sulfur from the gas-phase. At intermediate PDR depths $\left(A_{V}<8 \mathrm{mag}\right)$ the observed $\mathrm{H}_{2} \mathrm{~S}$ column densities do not require depletion of elemental (cosmic) sulfur abundances either. - We conclude that everywhere $\mathrm{s}-\mathrm{H}_{2} \mathrm{~S}$ grain mantles form (or formed) gas-phase $\mathrm{H}_{2} \mathrm{~S}$ will be present in detectable amounts. Independently of $n_{\mathrm{H}}$ and $G_{0}$, FUV-illuminated clouds produce roughly the same $\mathrm{H}_{2} \mathrm{~S}$ column density (a few $10^{14} \mathrm{~cm}^{-2}$ ) and $\mathrm{H}_{2} \mathrm{~S}$ peak abundances (a few $10^{-8}$ ). This agrees with the $\mathrm{H}_{2} \mathrm{~S}$ column densities derived in the Orion Bar and at the edges of mildly illuminated clouds. Deep inside molecular clouds ( $\left.A_{V}>8 \mathrm{mag}\right), \mathrm{H}_{2} \mathrm{~S}$ still forms by direct chemical desorption and photodesorption by secondary FUV photons. These processes alter the abundances of other S-bearing species and makes difficult to predict the dominant sulfur reservoir in cloud interiors.

In this study we focused on S-bearing hydrides. Still, many subtle details remain to be fully understood: radiative associations, electron recombinations, and formation of multiply sulfuretted molecules. For example, the low-temperature $\left(T_{k}<1000 \mathrm{~K}\right)$ rates of the radiative and dielectronic recombination of $\mathrm{S}^{+}$used in PDR models may still be not accurate enough (Badnell 1991). In addition, the main ice-mantle sulfur reservoirs are not fully constrained observationally. Thus, some of the narrative may be subject to speculation. Similarly, reactions of $\mathrm{S}^{+}$with abundant organic molecules desorbed from grains (such as $\mathrm{s}-\mathrm{H}_{2} \mathrm{CO}$, not considered in our study) may contribute to enhance the $\mathrm{H}_{2} \mathrm{~S}^{+}$abundance through gas-phase reactions (e.g., $\mathrm{S}^{+}+\mathrm{H}_{2} \mathrm{CO} \rightarrow \mathrm{H}_{2} \mathrm{~S}^{+}+\mathrm{CO}$; Prasad \& Huntress 1982). Future observations of the abundance and freeze out depths of the key ice carriers with JWST will clearly help in these fronts.
Acknowledgements. We warmly thank Prof. György Lendvay for interesting discussions and for sharing the codes related to their $\mathrm{S}\left({ }^{3} P\right)+\mathrm{H}_{2}\left({ }^{1} \Sigma_{g}^{+}, \mathrm{v}\right)$ PES. We thank Paul Dagdigian, François Lique, and Alexandre Faure for sharing their $\mathrm{H}_{2} \mathrm{~S}-\mathrm{H}_{2}, \mathrm{SH}^{+}-\mathrm{H}$, and $\mathrm{SH}^{+}-\mathrm{e}^{-}$inelastic collisional rate coefficients and for interesting discussions in Grenoble and Salamanca. We thank Helgi Hrodmarsson for sending his experimental SH photoionization cross section in tabulated format. We finally thank our referee, John H. Black, for encouraging and insightful suggestions. This paper makes use of the ALMA data ADS/JAO.ALMA\#2012.1.00352.S. ALMA is a partnership of ESO (representing its member states), NSF (USA), and NINS (Japan), together with NRC (Canada), and NSC and ASIAA (Taiwan), in cooperation with the Republic of Chile. The Joint ALMA Observatory is operated by ESO, AUI/NRAO, and NAOJ. It also includes IRAM $30 \mathrm{~m}$ telescope observations. IRAM is supported by INSU/CNRS (France), MPG (Germany), and IGN (Spain). We thank the staff at the IRAM30m telescope and the work of the USRA and NASA staff of the Armstrong Flight Research Center in Palmdale and of the Ames Research Center in Mountain View (California), and the Deutsches SOFIA Institut. We thank the Spanish MICIU for funding support under grants AYA2016-75066-C2-2-P, AYA2017-85111-P, FIS2017-83473-C2 PID2019-106110GB-I00, and PID2019-106235GB-I00 and the French-Spanish collaborative project PICS (PIC2017FR). We finally acknowledge computing time at Finisterrae (CESGA) under RES grant ACCT-2019-3-0004.

\section{References}

Aguado, A., Barragan, P., Prosmiti, R., et al. 2010, J. Chem. Phys., 133, 024306 Aguado, A. \& Paniagua, M. 1992, J. Chem. Phys., 96, 1265

Aguado, A., Tablero, C., \& Paniagua, M. 2001, Comput. Phys. Comm., 134, 97 Agúndez, M., Goicoechea, J. R., Cernicharo, J., Faure, A., \& Roueff, E. 2010, ApJ, 713, 662

Agúndez, M. \& Wakelam, V. 2013, Chemical Reviews, 113, 8710

Allers, K. N., Jaffe, D. T., Lacy, J. H., Draine, B. T., \& Richter, M. J. 2005, ApJ, 630,368

Anders, E. \& Grevesse, N. 1989, Geochim. Cosmochim. Acta, 53, 197

Andree-Labsch, S., Ossenkopf-Okada, V., \& Röllig, M. 2017, A\&A, 598, A2

Anicich, V. G. 2003, JPL Publication 03-19, 1-1194

Arab, H., Abergel, A., Habart, E., et al. 2012, A\&A, 541, A19

Asplund, M., Grevesse, N., Sauval, A. J., \& Scott, P. 2009, ARA\&A, 47, 481

Azzam, A. A. A., Yurchenko, S. N., Tennyson, J., Martin-Drumel, M.-A., \& Pirali, O. 2013, J. Quant. Spectr. Rad. Transf., 130, 341

Badnell, N. R. 1991, ApJ, 379, 356

Bally, J. 2008, Overview of the Orion Complex, ed. B. Reipurth, 459

Bañares, L., Aoiz, F. J., Honvault, P., Bussery-Honvault, B., \& Launay, J.-M. 2003, J. Chem. Phys., 118, 565

Bañares, L., Aoiz, F. J., Honvault, P., \& Launay, J.-M. 2004, J. Phys. Chem., 108, 1616

Black, J. H. 1998, Faraday Discussions, 109, 257

Bonnet, L. \& Rayez, J.-C. 1997, Chem. Phys. Lett., 277, 183

Bonnet, L. \& Rayez, J.-C. 2004, Chem. Phys. Lett., 397, 106

Brittain, A., Coolbroth, K., \& Boogert, A. 2020, in American Astronomical Society Meeting Abstracts, Vol. 236, American Astronomical Society Meeting Abstracts \#236, 247.08

Bron, E., Agúndez, M., Goicoechea, J. R., \& Cernicharo, J. 2018, ArXiv e-prints Bron, E., Le Bourlot, J., \& Le Petit, F. 2014, A\&A, 569, A100

Buckinghan, A. D. 1967, Adv. Chem. Phys., 12, 107

Burton, M. G., Hollenbach, D. J., \& Tielens, A. G. G. M. 1990, ApJ, 365, 620

Calmonte, U., Altwegg, K., Balsiger, H., et al. 2016, MNRAS, 462, S253

Cardelli, J. A., Clayton, G. C., \& Mathis, J. S. 1989, ApJ, 345, 245

Caselli, P., Keto, E., Bergin, E. A., et al. 2012, ApJ, 759, L37

Caselli, P., Keto, E., Pagani, L., et al. 2010, A\&A, 521, L29

Charnley, S. B. 1997, ApJ, 481, 396

Choi, Y., van der Tak, F. F. S., Bergin, E. A., \& Plume, R. 2014, A\&A, 572, L10

Collings, M. P., Anderson, M. A., Chen, R., et al. 2004, MNRAS, 354, 1133

Crockett, N. R., Bergin, E. A., Neill, J. L., et al. 2014, ApJ, 781, 114

Cruz-Diaz, G. A., Muñoz Caro, G. M., Chen, Y. J., \& Yih, T. S. 2014, A\&A, 562, A119

Cuadrado, S., Goicoechea, J. R., Cernicharo, J., et al. 2017, A\&A, 603, A124

Cuadrado, S., Goicoechea, J. R., Pilleri, P., et al. 2015, A\&A, 575, A82

Cuadrado, S., Goicoechea, J. R., Roncero, O., et al. 2016, A\&A, 596, L1

Cuadrado, S., Salas, P., Goicoechea, J. R., et al. 2019, A\&A, 625, L3

Dagdigian, P. J. 2019, MNRAS, 487, 3427

Dagdigian, P. J. 2019, J. Chem. Phys., 150, 084308

Dagdigian, P. J. 2020, MNRAS, 494, 5239

Dartois, E. 2005, Space Sci. Rev., 119, 293

Davidson, E. R. 1975, J. Comp. Phys., 17, 87

de Graauw, T., Helmich, F. P., Phillips, T. G., et al. 2010, A\&A, 518, L6

Desrousseaux, B., Lique, F., Goicoechea, J. R., Quintas-Sánchez, E., \& Dawes, R. 2021, A\&A, 645, A8 
Endres, C. P., Schlemmer, S., Schilke, P., Stutzki, J., \& Müller, H. S. P. 2016 , Journal of Molecular Spectroscopy, 327, 95

Esplugues, G. B., Cazaux, S., Meijerink, R., Spaans, M., \& Caselli, P. 2016, A\&A, 591, A52

Esplugues, G. B., Viti, S., Goicoechea, J. R., \& Cernicharo, J. 2014, A\&A, 567, A95

Farah, K., Muller-Plathe, F., \& Bohm, M. C. 2012, Chem. Phys. Chem., 13, 1127

Faure, A., Halvick, P., Stoecklin, T., et al. 2017, MNRAS, 469, 612

Freeman, A. \& Williams, D. A. 1982, Ap\&SS, 83, 417

Fuente, A., Cernicharo, J., Roueff, E., et al. 2016, A\&A, 593, A94

Fuente, A., Goicoechea, J. R., Pety, J., et al. 2017, ApJ, 851, L49

Fuente, A., Navarro, D. G., Caselli, P., et al. 2019, A\&A, 624, A105

Fuente, A., Rodrıguez-Franco, A., Garcı-Burillo, S., Martın-Pintado, J., \& Black, J. H. 2003, A\&A, 406, 899

Garrod, R. T., Wakelam, V., \& Herbst, E. 2007, A\&A, 467, 1103

Genzel, R. \& Stutzki, J. 1989, ARA\&A, 27, 41

Gerin, M., de Luca, M., Black, J., et al. 2010, A\&A, 518, L110

Gerin, M., Neufeld, D. A., \& Goicoechea, J. R. 2016, ARA\&A, 54, 181

Gibb, E. L., Whittet, D. C. B., Boogert, A. C. A., \& Tielens, A. G. G. M. 2004, ApJS, 151, 35

Godard, B. \& Cernicharo, J. 2013, A\&A, 550, A8

Godard, B., Falgarone, E., Gerin, M., et al. 2012, A\&A, 540, A87

Godard, B., Falgarone, E., \& Pineau des Forêts, G. 2014, A\&A, 570, A27

Goicoechea, J. R., Cernicharo, J., Karska, A., et al. 2012, A\&A, 548, A77

Goicoechea, J. R., Cuadrado, S., Pety, J., et al. 2017, A\&A, 601, L9

Goicoechea, J. R., Joblin, C., Contursi, A., et al. 2011, A\&A, 530, L16

Goicoechea, J. R. \& Le Bourlot, J. 2007, A\&A, 467, 1

Goicoechea, J. R., Pabst, C. H. M., Kabanovic, S., et al. 2020, A\&A, 639, A1

Goicoechea, J. R., Pety, J., Cuadrado, S., et al. 2016, Nature, 537, 207

Goicoechea, J. R., Pety, J., Gerin, M., Hily-Blant, P., \& Le Bourlot, J. 2009 A\&A, 498, 771

Goicoechea, J. R., Pety, J., Gerin, M., et al. 2006, A\&A, 456, 565

Goicoechea, J. R., Santa-Maria, M. G., Bron, E., et al. 2019, A\&A, 622, A91

Gómez-Carrasco, S. \& Roncero, O. 2006, J. Chem. Phys., 125, 054102

Graedel, T. E., Langer, W. D., \& Frerking, M. A. 1982, ApJS, 48, 321

Grozdanov, T. P. \& Solov'ev, E. A. 1982, J. Phys. B, 15, 1195

Guzmán, V. V., Goicoechea, J. R., Pety, J., et al. 2013, A\&A, 560, A73

Habart, E., Dartois, E., Abergel, A., et al. 2010, A\&A, 518, L116

Habing, H. J. 1968, Bull. Astron. Inst. Netherlands, 19, 421

Hama, T. Kouchi, A., \& Watanabe, N. 2016, Science, 351, 65

Hamilton, J. R., Faure, A., \& Tennyson, J. 2018, MNRAS, 476, 2931

Hasegawa, T. I. \& Herbst, E. 1993, MNRAS, 261, 83

Hatchell, J., Thompson, M. A., Millar, T. J., \& MacDonald, G. H. 1998, A\&A, 338,713

He, J., Shi, J., Hopkins, T., Vidali, G., \& Kaufman, M. J. 2015, ApJ, 801, 120

Heays, A. N., Bosman, A. D., \& van Dishoeck, E. F. 2017, A\&A, 602, A105

Heikkilä, A., Johansson, L. E. B., \& Olofsson, H. 1999, A\&A, 344, 817

Herbst, E., DeFrees, D. J., \& Koch, W. 1989, Mon. Not. R. Astyr. Soc., 237, 1057

Herpin, F., Marseille, M., Wakelam, V., Bontemps, S., \& Lis, D. C. 2009, A\&A, 504,853

Heyminck, S., Graf, U. U., Güsten, R., et al. 2012, A\&A, 542, L1

Hogerheijde, M. R., Jansen, D. J., \& van Dishoeck, E. F. 1995, A\&A, 294, 792

Holdship, J., Jimenez-Serra, I., Viti, S., et al. 2019, ApJ, 878, 64

Hollenbach, D., Kaufman, M. J., Bergin, E. A., \& Melnick, G. J. 2009, ApJ, 690, 1497

Hollenbach, D., Kaufman, M. J., Neufeld, D., Wolfire, M., \& Goicoechea, J. R. 2012, ApJ, 754, 105

Hollenbach, D. J. \& Tielens, A. G. G. M. 1997, ARA\&A, 35, 179

Hosokawa, T. \& Inutsuka, S.-i. 2006, ApJ, 646, 240

Howk, J. C., Sembach, K. R., \& Savage, B. D. 2006, ApJ, 637, 333

Hrodmarsson, H. R., Garcia, G. A., Nahon, L., Loison, J.-C., \& Gans, B. 2019 , Physical Chemistry Chemical Physics (Incorporating Faraday Transactions), 21,25907

Indriolo, N., Neufeld, D. A., Gerin, M., et al. 2015, ApJ, 800, 40

Ioppolo, S., Cuppen, H. M., Romanzin, C., van Dishoeck, E. F., \& Linnartz, H. 2008, ApJ, 686, 1474

Jansen, D. J., Spaans, M., Hogerheijde, M. R., \& van Dishoeck, E. F. 1995, A\&A, 303,541

Jiménez-Escobar, A. \& Muñoz Caro, G. M. 2011, A\&A, 536, A91

Jiménez-Serra, I., Zhang, Q., Viti, S., Martín-Pintado, J., \& de Wit, W. J. 2012, ApJ, 753, 34

Joblin, C., Bron, E., Pinto, C., et al. 2018, A\&A, 615, A129

Johnson, B. R. 1987, J. Chem. Phys., 86, 1445

Kaplan, K. F., Dinerstein, H. L., Oh, H., et al. 2017, ApJ, 838, 152

Karplus, M., Porter, R. N., \& Sharma, R. D. 1965, J. Chem. Phys., 43, 3259

Kirsanova, M. S. \& Wiebe, D. S. 2019, MNRAS, 486, 2525

Klisch, E., Klaus, T., Belov, S. P., et al. 1996, ApJ, 473, 1118

Kłos, J., Lique, F., \& Alexander, M. H. 2009, Chemical Physics Letters, 476, 135

Knizia, G., Adler, T. B., \& Werner, H. J. 2009, J. Chem. Phys., 130, 054104

Laas, J. C. \& Caselli, P. 2019, A\&A, 624, A108
Le Bourlot, J., Le Petit, F., Pinto, C., Roueff, E., \& Roy, F. 2012, A\&A, 541, A76

Le Petit, F., Nehmé, C., Le Bourlot, J., \& Roueff, E. 2006, ApJS, 164, 506

Lee, T. A. 1968, ApJ, 152, 913

Leurini, S., Rolffs, R., Thorwirth, S., et al. 2006, A\&A, 454, L47

Levine, R. D. \& Bernstein, R. B. 1987, Molecular Reaction Dynamics and Chemical Reactivity (Oxford University Press)

Lique, F., Zanchet, A., Bulut, N., Goicoechea, J. R., \& Roncero, O. 2020, A\&A, 638, A72

Lis, D. C., Bergin, E. A., Schilke, P., \& van Dishoeck, E. F. 2013, Journal of Physical Chemistry A, 117, 9661

Lucas, R. \& Liszt, H. S. 2002, A\&A, 384, 1054

Maiti, B., Schatz, G. C., \& Lendvay, G. 2004, Journal of Physical Chemistry A, 108,8772

Marconi, A., Testi, L., Natta, A., \& Walmsley, C. M. 1998, A\&A, 330, 696

Martín, S., Krips, M., Martín-Pintado, J., et al. 2011, A\&A, 527, A36

Martín, S., Mauersberger, R., Martín-Pintado, J., Henkel, C., \& García-Burillo, S. 2006, ApJS, 164,450

Martin-Drumel, M. A., Eliet, S., Pirali, O., et al. 2012, Chemical Physics Letters, 550,8

Melnick, G. J., Tolls, V., Goldsmith, P. F., et al. 2012, ApJ, 752, 26

Melnick, G. J., Tolls, V., Snell, R. L., et al. 2020, ApJ, 892, 22

Menten, K. M., Wyrowski, F., Belloche, A., et al. 2011, A\&A, 525, A77

Millar, T. J., Adams, N. G., Smith, D., Lindinger, W., \& Villinger, H. 1986, MNRAS, 221, 673

Millar, T. J. \& Herbst, E. 1990, Astron. Astrophys., 231, 466

Millar, T. J. \& Herbst, E. 1990, A\&A, 231, 466

Minissale, M. \& Dulieu, F. 2014, J. Chem. Phys., 141, 014304

Minissale, M., Dulieu, F., Cazaux, S., \& Hocuk, S. 2016, A\&A, 585, A24

Mumma, M. J., Weaver, H. A., \& Larson, H. P. 1987, A\&A, 187, 419

Nagy, T. \& Lendvay, G. 2017, J. Phys. Chem. Lett., 8, 4621

Nagy, Z., Choi, Y., Ossenkopf-Okada, V., et al. 2017, A\&A, 599, A22

Nagy, Z., Van der Tak, F. F. S., Ossenkopf, V., et al. 2013, A\&A, 550, A96

Navarro-Almaida, D., Le Gal, R., Fuente, A., et al. 2020, A\&A, 637, A39

Neufeld, D. A., Falgarone, E., Gerin, M., et al. 2012, A\&A, 542, L6

Neufeld, D. A., Godard, B., Gerin, M., et al. 2015, A\&A, 577, A49

Neufeld, D. A., Goicoechea, J. R., Sonnentrucker, P., et al. 2010, A\&A, 521, L10

Neufeld, D. A., Nisini, B., Giannini, T., et al. 2009, ApJ, 706, 170

Oba, Y., Tomaru, T., Lamberts, T., Kouchi, A., \& Watanabe, N. 2018, Nature Astronomy, 2, 228

O’Dell, C. R. 2001, ARA\&A, 39, 99

Pabst, C., Higgins, R., Goicoechea, J. R., et al. 2019, Nature, 565, 618

Pabst, C. H. M., Goicoechea, J. R., Teyssier, D., et al. 2020, A\&A, 639, A2

Palumbo, M. E., Geballe, T. R., \& Tielens, A. G. G. M. 1997, ApJ, 479, 839

Pankonin, V. \& Walmsley, C. M. 1978, A\&A, 64, 333

Parikka, A., Habart, E., Bernard-Salas, J., et al. 2017, A\&A, 599, A20

Pellegrini, E. W., Baldwin, J. A., Ferland, G. J., Shaw, G., \& Heathcote, S. 2009, ApJ, 693, 285

Peterson, K. A., Adler, T. B., \& Werner, H. J. 2008, J. Chem. Phys., 128, 084102 Pineau des Forets, G., Flower, D. R., Hartquist, T. W., \& Dalgarno, A. 1986, MNRAS, 220, 801

Prasad, S. S. \& Huntress, W. T., J. 1980, ApJS, 43, 1

Prasad, S. S. \& Huntress, W. T., J. 1982, ApJ, 260, 590

Putaud, T., Michaut, X., Le Petit, F., Roueff, E., \& Lis, D. C. 2019, A\&A, 632, A8

Qu, C. \& Bowman, J. M. 2016, J. Phys. Chem. A, 120, 4988

Rivière-Marichalar, P., Fuente, A., Goicoechea, J. R., et al. 2019, A\&A, 628, A16

Roelfsema, P. R., Helmich, F. P., Teyssier, D., et al. 2012, A\&A, 537, A17

Roncero, O., Zanchet, A., \& Aguado, A. 2018, Phys. Chem. Chem. Phys., 20, 25951

Rosenthal, D., Bertoldi, F., \& Drapatz, S. 2000, A\&A, 356, 705

Sandford, S. A. \& Allamandola, L. J. 1988, Icarus, 76, 201

Sanz-Sanz, C., Roncero, O., Paniagua, M., \& Aguado, A. 2013, J. Chem. Phys., 139,184302

Shiozaki, T. \& Werner, H.-J. 2013, Mol. Phys., 111, 607, mRCI-F12

Smirnov, G. T., Sorochenko, R. L., \& Walmsley, C. M. 1995, A\&A, 300, 923

Smith, R. G. 1991, MNRAS, 249, 172

Sofia, U. J., Lauroesch, J. T., Meyer, D. M., \& Cartledge, S. I. B. 2004, ApJ, 605, 272

Stecher, T. P. \& Williams, D. A. 1972, ApJ, 177, L141

Sternberg, A. \& Dalgarno, A. 1995, ApJS, 99, 565

Stoerzer, H., Stutzki, J., \& Sternberg, A. 1995, A\&A, 296, L9

Stowe, G. F., Schultz, R. H., Wright, C. A., \& Armentrout, P. B. 1990, Int. J. Mass Spectrom. Ion Proc., 100, 377

Tablero, C., Aguado, A., \& Paniagua, M. 2001, Comput. Phys. Comm., 140, 412

Taquet, V., Furuya, K., Walsh, C., \& van Dishoeck, E. F. 2016, MNRAS, 462, S 99

Tercero, B., Cernicharo, J., Pardo, J. R., \& Goicoechea, J. R. 2010, A\&A, 517, A96 
Tieftrunk, A., Pineau des Forets, G., Schilke, P., \& Walmsley, C. M. 1994, A\&A, 289, 579

Tielens, A. G. G. M. 2010, The Physics and Chemistry of the Interstellar Medium

Tielens, A. G. G. M. \& Hagen, W. 1982, A\&A, 114, 245

Tielens, A. G. G. M. \& Hollenbach, D. 1985, ApJ, 291, 722

Tielens, A. G. G. M., Meixner, M. M., van der Werf, P. P., et al. 1993, Science, 262,86

Turner, B. E. 1996, ApJ, 468, 694

van der Tak, F. F. S., Black, J. H., Schöier, F. L., Jansen, D. J., \& van Dishoeck, E. F. 2007 , A\&A, 468,627

van der Tak, F. F. S., Boonman, A. M. S., Braakman, R., \& van Dishoeck, E. F. 2003, A\&A, 412, 133

van der Tak, F. F. S., Nagy, Z., Ossenkopf, V., et al. 2013, A\&A, 560, A95

van der Werf, P. P., Goss, W. M., \& O'Dell, C. R. 2013, ApJ, 762, 101

van der Werf, P. P., Stutzki, J., Sternberg, A., \& Krabbe, A. 1996, A\&A, 313, 633

van der Wiel, M. H. D., van der Tak, F. F. S., Ossenkopf, V., et al. 2009, A\&A, 498,161

van Dishoeck, E. F. 2004, ARA\&A, 42, 119

Velilla, L., Lepetit, B., Aguado, A., Beswick, J., \& Paniagua, M. 2008, J. Chem. Phys., 129, 084307

Vidal, T. H. G., Loison, J.-C., Jaziri, A. Y., et al. 2017, MNRAS, 469, 435

Wakelam, V., Loison, J. C., Mereau, R., \& Ruaud, M. 2017, Molecular Astrophysics, 6,22

Walmsley, C. M., Natta, A., Oliva, E., \& Testi, L. 2000, A\&A, 364, 301

Werner, H. J. \& Knowles, P. J. 1988a, J. Chem. Phys., 89, 5803

Werner, H. J. \& Knowles, P. J. 1988b, Chem. Phys. Lett., 145, 514

Werner, H.-J., Knowles, P. J., Knizia, G., Manby, F. R., \& Schütz, M. 2012, WIREs Comput Mol Sci, 2, 242

Wyrowski, F., Schilke, P., Hofner, P., \& Walmsley, C. M. 1997, ApJ, 487, L171

Yamamura, I., Kawaguchi, K., \& Ridgway, S. T. 2000, ApJ, 528, L33

Young, E. T., Becklin, E. E., Marcum, P. M., et al. 2012, ApJ, 749, L17

Zanchet, A., Agúndez, M., Herrero, V. J., Aguado, A., \& Roncero, O. 2013a, AJ, 146,125

Zanchet, A., del Mazo, P., Aguado, A., et al. 2018, PCCP, 20, 5415

Zanchet, A., Godard, B., Bulut, N., et al. 2013b, ApJ, 766, 80

Zanchet, A., Lique, F., Roncero, O., Goicoechea, J. R., \& Bulut, N. 2019, A\&A, 626, A103

Zanchet, A., Roncero, O., González-Lezana, T., et al. 2009, Journal of Physical Chemistry A, 113, 14488

Zhou, J., Zhao, Y., Hansen, C. S., et al. 2020, Nature Communications, 11, 1547 


\section{Appendix $\mathrm{A}: \mathrm{H}_{2} \mathrm{~S}^{+}$formation and destruction}

In this Appendix we give details about how we calculated the $\mathrm{H}_{2}$ vibrational-state-dependent rates of reaction (2) and of the reverse reaction, the destruction of $\mathrm{H}_{2} \mathrm{~S}^{+}\left({ }^{2} A^{\prime}\right)$ by reactive collisons with $\mathrm{H}\left({ }^{2} S\right)$ atoms (summarized in Fig. A.1).

We first built a full dimensional potential energy surface (PES) of the triplet $\mathrm{H}_{3} \mathrm{~S}^{+}\left({ }^{3} \mathrm{~A}\right)$ system by fitting more than 150,000 $a b$ initio points, including the long range interactions in the reactants and products channels. The main topological features of the PES are summarized in the minimum energy path between reactants and products (see middle panel of Fig. 9). These $a b$ initio points were calculated with an explicitly correlated restricted coupled cluster including a single, double, and (perturbatively) triple excitations (RCCSD(T)-F12a) method (Knizia et al. 2009). The analytical fit has a overall rms error of $\simeq 0.01 \mathrm{eV}$ (Fig. A.2). Appendix A.1 provides more details.

Reaction (2) is endothermic by $0.672 \mathrm{eV}$, and the PES of the triplet state shows two shallow wells in the $\mathrm{H}_{2}+\mathrm{SH}^{+}$entrance channel (named ${ }^{3} W_{1 a}$ and ${ }^{3} W_{1 b}$, with a depth of $\simeq 0.118 \mathrm{eV}$ ) and another one near the $\mathrm{H}+\mathrm{H}_{2} \mathrm{~S}^{+}$products (named ${ }^{3} W_{2}$, with a depth of $0.08 \mathrm{eV}$ ). Between the reactants and products wells there is a saddle point, with an energy of $0.601 \mathrm{eV}$. This saddle point, slightly below the products, has a geometry similar to ${ }^{3} W_{2}$ in which the $\mathrm{H}-\mathrm{H}$ distance is strongly elongated compared to that of $\mathrm{H}_{2}$. These features are also present in the maximum multiplicity PES of reactions $\mathrm{H}_{2}+\mathrm{S}^{+}\left({ }^{4} S\right)$ and $\mathrm{H}_{2}+\mathrm{H}_{2} \mathrm{~S}^{+}\left({ }^{2} A\right)$ (see Fig. 9). We determine the state-dependent rates of reaction (2) and of the reverse reaction using a quasi-classical trajectory (QCT) method on our ground triplet PES. We provide more details on how the reactive cross sections for fixed collision energies were calculated in Appendix. A.2.

The formation rate of $\mathrm{H}_{2} \mathrm{~S}^{+}$from $\mathrm{H}_{2}(v=0)$ is very slow. For $\mathrm{H}_{2}(v=1)$, the rate constant significantly increases at $\approx 500 \mathrm{~K}$, corresponding with the opening of the $\mathrm{H}_{2} \mathrm{~S}^{+}+\mathrm{H}$ threshold. At this point, it is important to consider the zero-point energy (ZPE) of the products (see next section for details). For $\mathrm{H}_{2}(v=2)$ and $\mathrm{H}_{2}(v=3)$, reaction rates are faster, close to the Langevin limit. Finally, the $\mathrm{H}_{2} \mathrm{~S}^{+}$destruction rate constant is very similar to that of its formation from $\mathrm{H}_{2}(v=2)$. In Appendix A.3 we provide more information about the destruction of $\mathrm{H}_{n} \mathrm{~S}^{+}$ions through radiative association and spin flip mechanisms.

\section{Appendix A.1: Ab initio calculations and PES}

Dagdigian (2019) presented a PES for the $\mathrm{SH}^{+}-\mathrm{H}_{2}$ system that includes 4-dimensions and is based on $\operatorname{RCCSD}(\mathrm{T})$-F12a ab initio calculations. This PES was used to study $\mathrm{SH}^{+}-\mathrm{H}_{2}$ inelastic collisions using a rigid rotor approach in which the two diatomic molecules are kept fixed at their equilibrium distances. However, in order to study the reactivity of the collision, the two diatomic distances have to be included to account for the breaking and formation of new bonds.

Reaction (2) corresponds to a triplet state $\mathrm{H}_{3} \mathrm{~S}^{+}\left({ }^{3} A\right)$. The $\mathrm{H}_{2} \mathrm{~S}^{+}\left({ }^{2} A^{\prime}\right)+\mathrm{H}\left({ }^{2} S\right)$ products can form a triplet and a singlet state. The triplet state can lead to the destruction of $\mathrm{H}_{2} \mathrm{~S}^{+}$through reaction with $\mathrm{H}$ atoms. The singlet state, however, produces very excited states of the reactants. Thus, it only leads to inelastic collisions but not not to the destruction of $\mathrm{H}_{2} \mathrm{~S}^{+}\left({ }^{2} A^{\prime}\right)$. In consequence, here we only consider the ground triplet electronic state of the system. In addition, the $\mathrm{H}_{3}^{+}+\mathrm{S}\left({ }^{3} P\right)$ channel is about $2.4 \mathrm{eV}$ above the $\mathrm{H}_{2}+\mathrm{SH}^{+}$asymptote, and will not be included in the present study.

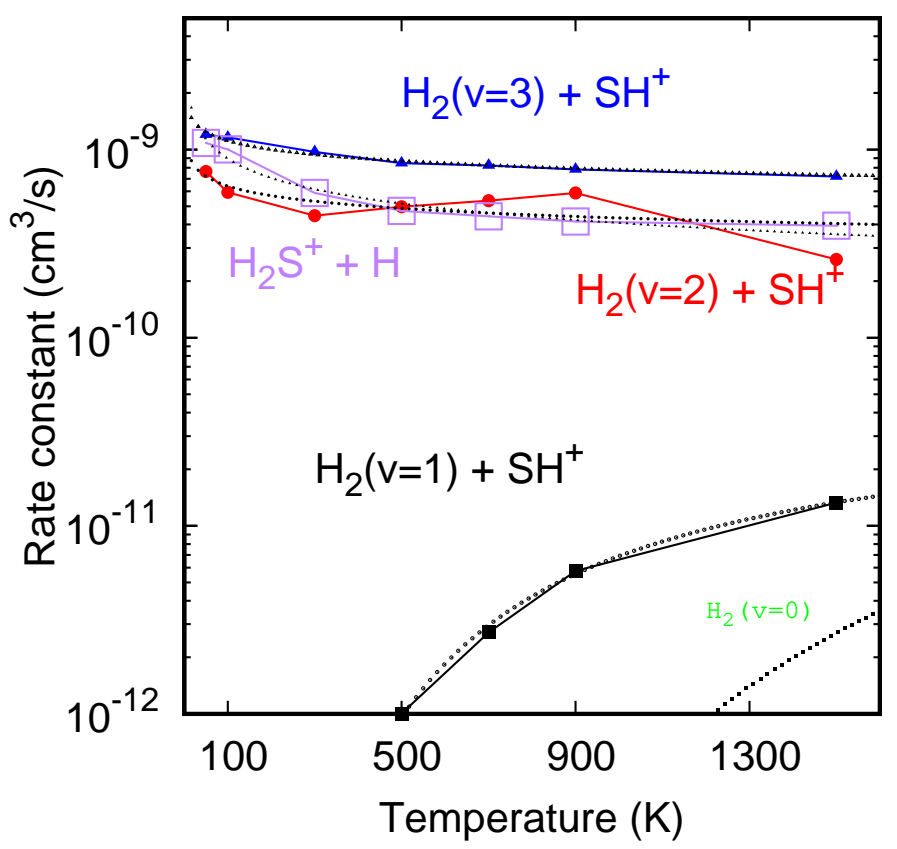

Fig. A.1. Calculated rate constants as a function of temperature (for translation and rotation) for $\mathrm{SH}^{+}(v=0, j=0)+\mathrm{H}_{2}(v=1,2,3, j=0)$ and $\mathrm{H}_{2} \mathrm{~S}^{+}(v=0, j=0)+\mathrm{H}$ reactions (lavender) using ZPE corrected QCT method. Dotted curves are fits of the form $k(T)=\alpha(T / 300)^{\beta} \exp (-\gamma / T)$. Rate coefficients are listed in Table 1.

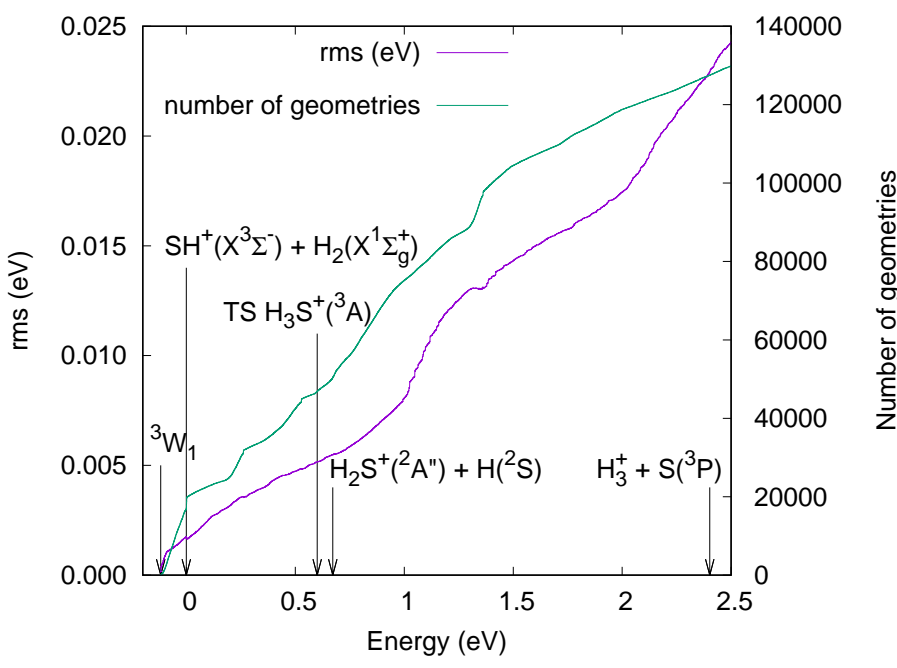

Fig. A.2. Rms error as a function of total energy, showing the number of $a b$ initio points used to evaluate the error in the PES calculation. Arrows indicate selected critical points in the PES and provide an estimate of the error in each region. TS means transition state.

In order to study the regions where several electronic states intersect, we performed a explicitly correlated internally contracted multireference configuration interaction (ic-MRCI-F12) calculation (Shiozaki \& Werner 2013; Werner \& Knowles 1988a,b) including the Davidson correction (icMRCI-F12+Q; Davidson 1975). The ic-MRCI-F12 calculations were carried out using state-averaged complete active space self-consistent field (SA-CASSCF) orbitals with all the CAS configurations as the reference configuration state functions. We used a triple zeta correlation consistent basis set for explicitly correlated wave functions (cc-pVTZ-F12; Peterson et al. 2008). In order to avoid orbital flipping between core and valence orbitals. SA-CASSCF calcula- 
tions with three lowest triplet states were carried out including the core and valence orbitals as active space (18 electrons in 11 orbitals). For the ic-MRCI-F12 calculation, the core orbitals was kept doubly occupied, resulting in about $2.5 \times 10^{6}\left(9 \times 10^{7}\right)$ contracted (uncontracted) configurations. All $a b$ initio calculations were performed with MOLPRO (Werner et al. 2012).

Our ic-MRCI-F12 calculations show that the crossings with electronic excited states are $2 \mathrm{eV}$ above the energy of the reactants. The energy interval below $2 \mathrm{eV}$ is enough to study reaction 2. In these low-energy regions, RCCSD(T)-F12a calculations were also performed. They are in good agreement with the ic-MRCI-F12 results and the $t_{1}$ diagnostic is always below 0.03 . This allows us to conclude that for energies below $2 \mathrm{eV}$, the RCCSD(T)-F12a method performs well, presents a simple convergence, and being size consistent, is well adapted to the present case. This method is the same one employed in the inelastic collision calculations by Dagdigian (2019).

We performed extensive RCCSD(T)-F12a calculations in all accessible regions to properly describe the six-dimensional phase space. $150000 a b$ initio points were fitted to a multidimensional analytic function, that generates the six-dimensional PES represented as

$H=H^{d i a b}+H^{M B}$

(Aguado et al. 2010; Sanz-Sanz et al. 2013; Zanchet et al. 2018; Roncero et al. 2018), where $H^{\text {diab }}$ is an electronic diabatic matrix in which each diagonal matrix element describes a rearrangement channel - six in this case, three equivalent for $\mathrm{SH}^{+}+\mathrm{H}_{2}$ channels, and three equivalent for $\mathrm{H}_{2} \mathrm{~S}^{+}+\mathrm{H}$ fragments (we omitted the $\mathrm{H}_{3}^{+}+\mathrm{S}$ channel) - as an extension of the reactive force field approach (Farah et al. 2012). In each diagonal term, the molecular fragments $\left(\mathrm{SH}^{+}, \mathrm{H}_{2}\right.$ and $\left.\mathrm{H}_{2} \mathrm{~S}^{+}\right)$are described by 2 or 3 body fits (Aguado \& Paniagua 1992), and the interaction among them is described by a sum of atom-atom terms plus the long range interaction. The non diagonal terms of $H^{\text {diab }}$ are described as previously (Zanchet et al. 2018; Roncero et al. 2018) and the parameters are fitted to approximately describe the saddle points along the minimum energy path in the right geometry.

In the reactants channel, the leading long range interaction $\mathrm{SH}^{+}\left(X^{3} \Sigma^{-}\right)+\mathrm{H}_{2}\left(X^{1} \Sigma_{g}^{+}\right)$corresponds to charge-quadrupole and charge-induced dipole interactions (Buckinghan 1967):

$$
\begin{gathered}
V_{\text {charge }}\left(\mathbf{r}_{H H}, \mathbf{R}\right)=\Theta_{2}\left(r_{H H}\right) P_{2}\left(\cos \theta_{2}\right) R^{-3} \\
-\left[\frac{1}{2} \alpha_{0}\left(r_{H H}\right)+\frac{1}{3}\left(\alpha_{\|}\left(r_{H H}\right)-\alpha_{\perp}\left(r_{H H}\right)\right) P_{2}\left(\cos \theta_{2}\right)\right] R^{-4}(\text { A. } 2)
\end{gathered}
$$

and the dipole-quadrupole interactions (Buckinghan 1967):

$$
\begin{gathered}
V_{\text {dipole }}\left(\mathbf{r}_{S H}, \mathbf{r}_{H H}, \mathbf{R}\right)=3 \mu_{1}\left(r_{S H}\right) \Theta_{2}\left(r_{H H}\right) \\
\times \quad\left[\cos \theta_{1} P_{2}\left(\cos \theta_{2}\right)+\sin \theta_{1} \sin \theta_{2} \cos \theta_{2} \cos \phi\right] R^{-4},
\end{gathered}
$$

where $\Theta_{2}\left(r_{H H}\right)$ is the cuadrupole moment of $\mathrm{H}_{2}\left(X^{1} \Sigma_{g}^{+}\right), \alpha_{0}\left(r_{H H}\right)$, $\alpha_{\|}\left(r_{H H}\right)$, and $\alpha_{\perp}\left(r_{H H}\right)$ are the average, parallel, and perpendicular polarizabilities of $\mathrm{H}_{2}\left(X^{1} \Sigma_{g}^{+}\right)$, respectively, and $\mu_{1}\left(r_{S H}\right)$ is the dipole moment of $\mathrm{SH}^{+}\left(X^{3} \Sigma^{-}\right) . P_{2}(\cos \theta)$ represents the Legendre polynomial of degree 2 . The dependence of the molecular properties of $\mathrm{H}_{2}$ with the interatomic distance $r_{H H}$ is obtained from Velilla et al. (2008). The dipole moment of $\mathrm{SH}^{+}$depends on the origin of coordinates. Since $\mathrm{SH}^{+}\left(X^{3} \Sigma^{-}\right)$dissociates in $\mathrm{S}^{+}\left({ }^{4} S\right)+\mathrm{H}\left({ }^{2} S\right)$, we select the origin of coordinates in the $S$ atom, so that the dipole moment tends to zero when $\mathrm{R}$ goes to infinity.

In the products channel, the long range interaction $\mathrm{H}_{2} \mathrm{~S}^{+}\left(X^{2} A^{\prime \prime}\right)+\mathrm{H}\left({ }^{2} S\right)$ corresponds to the isotropic charge-
Table A.1. RCCSD(T)-F12a and fit stationary points on the PES.

\begin{tabular}{llrr}
\hline \hline Stationary point & Geometry & Energy/cm & Energy/eV \\
\hline Reactants & $\mathrm{SH}^{+}+\mathrm{H}_{2}$ & 0.0 & 0.0 \\
Minimum 1 & $\mathrm{SH}^{+}-\mathrm{H}_{2}$ & -950.2 & -0.1178 \\
TS12 & $\mathrm{SH}^{+} \cdot \cdot \mathrm{H}_{2}$ & -579.5 & -0.0719 \\
Minimum 2 & $\mathrm{SH}^{+}-\mathrm{H}_{2}$ & -937.9 & -0.1163 \\
TS13 & $\mathrm{SH}^{+} \cdot \cdot \mathrm{H} \cdot \cdot \mathrm{H}$ & 4843.9 & 0.6006 \\
Minimum 3 & $\mathrm{H}_{2} \mathrm{~S}^{+}-\mathrm{H}$ & 4766.5 & 0.5910 \\
Products & $\mathrm{H}_{2} \mathrm{~S}^{+}+\mathrm{H}$ & 5422.3 & 0.6723 \\
\hline
\end{tabular}

Table A.2. $E_{v}$ of reactants and products, and adiabatic switching energies for the QCT initial conditions.

\begin{tabular}{llr}
\hline System(vibration) & Exact $E_{v}(\mathrm{eV})$ & AS energy $(\mathrm{eV})$ \\
\hline $\mathrm{H}_{2}(v=0)$ & 0.270 & 0.269 \\
$\mathrm{H}_{2}(v=1)$ & 0.786 & 0.785 \\
$\mathrm{H}_{2}(v=2)$ & 1.272 & 1.272 \\
$\mathrm{H}_{2}(v=3)$ & 1.735 & 1.730 \\
$\mathrm{SH}^{+}(v=0)$ & 0.157 & 0.157 \\
$\mathrm{H}_{2} \mathrm{~S}^{+}(v=0)$ & 0.389 & 0.388 \\
\hline
\end{tabular}

induced dipole and charge-induced quadrupole dispersion terms

$$
V_{\mathrm{disp}}(R)=-\frac{9}{4} R^{-4}-\frac{15}{4} R^{-6}
$$

These long range terms diverge at $R=0$. To avoid this behavior, we replace $R$ by $\mathcal{R}$ :

$$
\mathcal{R}=R+R_{0} e^{-\left(R-R_{e}\right)} \quad \text { with } \quad R_{0}=10 \mathrm{bohr} .
$$

In Eq. (A.1), $H^{M B}$ is the many-body term, which is described by permutationaly invariant polynomials following the method of Aguado an collaborators (Aguado \& Paniagua 1992; Tablero et al. 2001; Aguado et al. 2001). This many-body term improves the accuracy of the PES, especially in the region of the reaction barriers (as shown in Fig. 9). Features of the stationary points are listed in Table A.1.

\section{Appendix A.2: Determination of reactive collision rates}

We studied the reaction dynamics using a quasi-classical trajectory (QCT) method with the code miQCT (Zanchet et al. 2018; Roncero et al. 2018). In this method, the initial vibrational energy of the reactants is included using the adiabatic switching method (AS) (Grozdanov \& Solov'ev 1982; Johnson 1987; Qu \& Bowman 2016; Nagy \& Lendvay 2017). Energies are listed in Table A.2. The initial distance between the center-of-mass of the reactants $\left(\mathrm{H}_{2}+\mathrm{SH}^{+}\right.$or $\left.\mathrm{H}_{2} \mathrm{~S}^{+}+\mathrm{H}\right)$ is set to $85 \mathrm{bohr}$, and the initial impact parameter is set randomly within a disk, the radius of which is set according to a capture model (Levine \& Bernstein 1987) using the corresponding long-range interaction. The orientation among the two reactants is set randomly.

A first exploration of the reaction dynamics is done at fixed collision energy, for $\mathrm{H}_{2}(v=0,1,2,3)+\mathrm{SH}^{+}(v=0)$ and $\mathrm{H}+\mathrm{H}_{2} \mathrm{~S}^{+}(v=0)$, and the reactive cross section is calculated as in Karplus et al. (1965)

$\sigma_{v j}(E)=\pi b_{\max }^{2} P_{r}(E) \quad$ with $\quad P_{r}(E)=\frac{N_{r}}{N_{t o t}}$, 


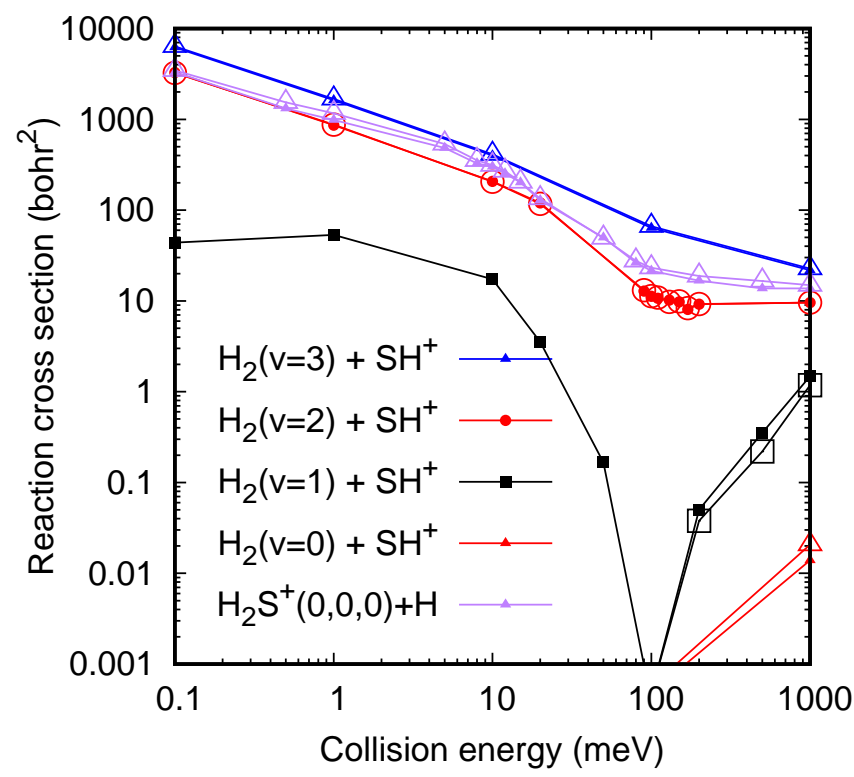

Fig. A.3. Reaction cross section (in bohr ${ }^{2}$ ) as a function of collision energy (in meV) for the $\mathrm{SH}^{+}(v=0, j=0)+\mathrm{H}_{2}(v=1,2,3, j=0)$ and $\mathrm{H}_{2} \mathrm{~S}^{+}(v=0, j=0)+\mathrm{H}$ collisions. Filled symbols are obtained counting all trajectories leading to products, while open symbols correspond to the ZPE corrected ones.

where $N_{t}$ is the maximum number of trajectories with initial impact parameter lower than $b_{\max }$, the maximum impact parameter for which the reaction takes place, and $N_{r}$ is the number of trajectories leading to products. Fig. A.2 shows results for $N_{t}>20000$ and all energies and initial reactant and vibrational states.

For the $\mathrm{SH}^{+}(v=0, j=0)+\mathrm{H}_{2}(v, j=0)$ reaction there is a strong dependence on the initial vibrational state. For $\mathrm{H}_{2}(v=0)$, there is nearly no reactive event, and only at $1 \mathrm{eV}$ there are some reactive trajectories. For $\mathrm{H}_{2}(v=2$ and 3$)$, however, the reaction shows a relatively large cross section, that decreases with increasing collision energy, as expected for exoergic reactions. Energies below $10-100 \mathrm{meV}$ are dominated by long range interactions, leading to an increase in the maximum impact parameter, $b_{\max }$, consistent with the variation of the cross section.

Reaction $\mathrm{SH}^{+}(v=0, j=0)+\mathrm{H}_{2}(v=1, j=0)$ shows an unexpected behavior that deserves some discussion. At energies below $40 \mathrm{meV}$, the cross section is large and decreases with increasing energy. In the 40-200 meV range, the reactive cross section drops to zero, showing a threshold at $200 \mathrm{meV}$ that is consistent with the endothermicity of the reaction.

In order to analyze the reaction mechanism for $\mathrm{H}_{2}(v=1)$ below $40 \mathrm{meV}$, we carried out an extensive analysis of the trajectories. A typical one is presented in Fig. A.4 for $10 \mathrm{meV}$. The $\mathrm{H}_{2}$ and $\mathrm{SH}^{+}$reactants are attracted to each other by long range interactions, until they get trapped in the ${ }^{3} W_{1}$ wells, as it is shown by the evolution of $R$, the distance between center-of-mass of the two molecules. The trapping lasts for $8 \mathrm{ps}$, thus allowing several collisions between $\mathrm{H}_{2}$ and $\mathrm{SH}^{+}$and permitting the energy transfer between them. The $\mathrm{H}_{2}$ molecule ultimately breaks, and leaves $\mathrm{SH}^{+}$with less vibrational energy. This can be inferred from the decrease in the amplitudes of the $\mathrm{SH}^{+}$distance. The energy of the $\mathrm{H}_{2} \mathrm{~S}^{+}$product is below the ZPE (see Table A.2). This is a clear indication of ZPE leakage in the QCT method, due to the energy transfer promoted by the long-lived collision complex.

Several methods exist that correct the ZPE leakage. One is the gaussian binning (Bonnet \& Rayez 1997, 2004; Bañares et al. 2003, 2004). Here we have applied a simplification of this method,

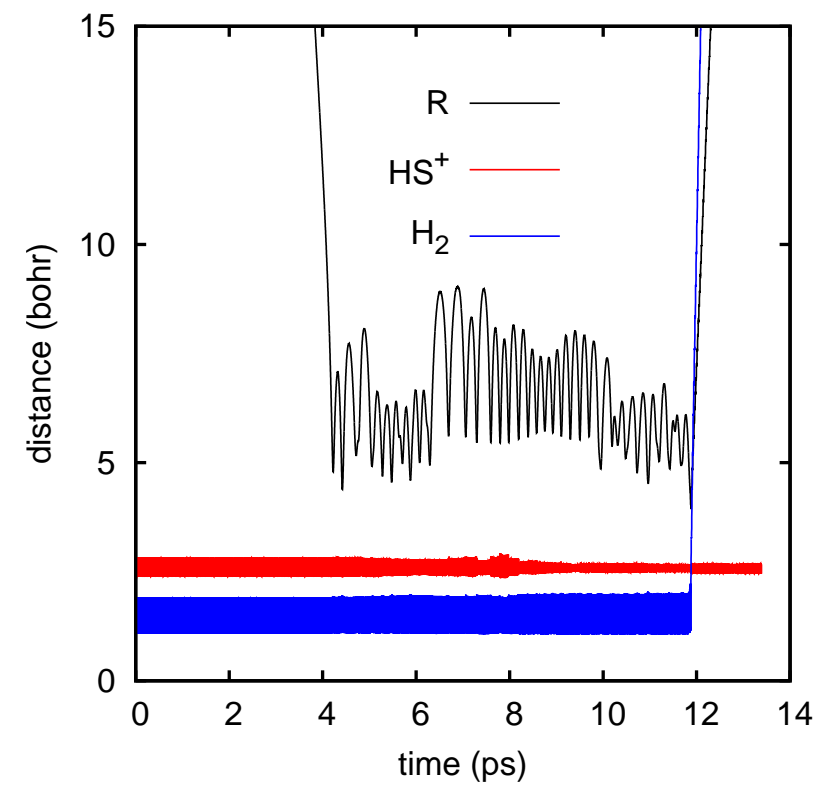

Fig. A.4. $\mathrm{H}-\mathrm{H}, \mathrm{SH}^{+}$and $R$ distances (in bohr) versus time (in ps), for a typical reactive trajectory for the $\mathrm{SH}^{+}(v=0, j=0)+\mathrm{H}_{2}(v=1, j=0)$ collision at $10 \mathrm{meV}$.

which assigns a weight $(w)$ for each trajectory as

$w=\left\{\begin{array}{ccc}1 & \text { for } & E_{v i b}>Z P E \\ e^{-\gamma\left(E_{v i b}-Z P E\right)^{2}} & \text { for } & E_{v i b}<Z P E\end{array}\right.$,

where $E_{v i b}$ is the vibrational energy of reactants (adding those of $\mathrm{H}_{2}$ and $\mathrm{SH}^{+}$) or $\mathrm{H}_{2} \mathrm{~S}^{+}$products at the end of each trajectory. These new weights are used to calculate $N_{r}$ and $\mathrm{N}_{t o t}$ in Eq. A.4. ZPE-corrected results are shown in Fig. A.3 with open symbols. This plot shows that all values are nearly the same as those calculated simply by counting trajectories as an integer (as done in the normal binning method; see filled symbols in Fig. A.3). The only exception is the case of $\mathrm{SH}^{+}+\mathrm{H}_{2}(v=1)$ below $400 \mathrm{meV}$, which becomes zero when considering the ZPE of fragments at the end of the trajectories.

The reaction thermal rate in specific initial vibrational state of reactants are calculated running a minimum of $10^{5}$ trajectories per temperature, with fixed vibrational states of reactants, assuming a Boltzmann distribution over translational and rotational degrees of freedom, and following the ZPE-corrected method as:

$k_{v}(T)=\sqrt{\frac{8 k_{B} T}{\pi \mu}} \quad \pi b_{\max }^{2}(T) \quad P_{r}(T)$.

The results of these calculations are shown in Fig. A.1.

\section{Appendix A.3: On the radiative associations of $\mathrm{H}_{n} \mathrm{~S}^{+}$}

Herbst et al. (1989) and Millar \& Herbst (1990) proposed that the radiative association $\mathrm{H}_{n} \mathrm{~S}^{+}+\mathrm{H}_{2} \rightarrow \mathrm{H}_{n+1} \mathrm{~S}^{+}+h v$ is viable process at low gas temperatures. Although this chemical route is widely used in astrochemical models, here we question the viability of this process. The lower multiplicity (L) PESs of $\mathrm{H}_{2} \mathrm{~S}^{+}\left({ }^{2} A^{\prime \prime}\right)$ and $\mathrm{H}_{3} \mathrm{~S}^{+}\left({ }^{1} A\right)$ are $\mathrm{L}=1 / 2$ and 0 respectively. These are shown in Fig. 9, together with the minimum multiplicity electronic state of $\mathrm{H}_{4} \mathrm{~S}^{+}$(bottom panel). This state does not have a deep well or any higher multiplicity state that could connect to higher states of reactants and products.

For of $\mathrm{H}_{3} \mathrm{~S}^{+}$formation through radiative association, this process assumes that a $\mathrm{H}_{3} \mathrm{~S}^{+}\left({ }^{3} A\right)^{*}$ complex forms in a triplet state, the 
high spin state $\mathrm{H}$ considered here. According to our calculations, such a complex is formed after low-energy $\mathrm{H}_{2}(v=0,1)+\mathrm{SH}^{+}$ reactions (below $40 \mathrm{meV}$ ). The complex is formed in the ${ }^{3} W_{1}$ well, corresponding to geometries very far from those of the low spin well, the ${ }^{1} W$ well. Therefore, a radiative spin flip and decay through phosphorescence is not possible. Herbst et al. (1989) proposed a second step, in which the spin flips from the triplet to the singlet state, followed by a radiative association, finally leading to the $\mathrm{H}_{3} \mathrm{~S}^{+}\left({ }^{1} A\right)$ product.

The origin of the spin flip must be the spin-orbit couplings, very relevant for S-bearing species, that favor the spin transition when singlet and triplet states are close in energy. Using the PESs calculated here, the lowest crossing region is at $\simeq 0.25 \mathrm{eV}$, very close to that of $\mathrm{H}_{2}(v=0)$. At low temperatures, the $\mathrm{H}_{3} \mathrm{~S}^{+}\left({ }^{3} A\right)^{*}$ complex formed by $\mathrm{H}_{2}(v=0)+\mathrm{SH}^{+}$reactions might allow a transition between the two electronic states with different spin. However, the spin flip probability is proportional to the square of the overlap $\left|\left\langle\mathrm{H}_{3} \mathrm{~S}^{+}\left({ }^{3} A\right)^{*} \mid \mathrm{H}_{3} \mathrm{~S}^{+}\left({ }^{1} A\right)^{*}\right\rangle\right|^{2}$. This probability is very small because the two wells, ${ }^{3} W_{1}$ and ${ }^{1} W_{1}$, correspond to very different geometries. In consequence, we conclude that this radiative association mechanism must be negligible, especially at the high gas temperatures of PDR edges where the $\mathrm{H}_{3} \mathrm{~S}^{+}\left({ }^{3} A\right)^{*}$ complex is not formed.

As an alternative, a spin flip in a direct collision (not forming a $\mathrm{H}_{3} \mathrm{~S}^{+}\left({ }^{3} A\right)^{*}$ complex) may be more efficient and should be further investigated. Indeed, experimental measurements of the $\mathrm{S}^{+}\left({ }^{4} S\right)+\mathrm{H}_{2}(v=0)$ cross section show a maximum at about $1 \mathrm{eV}$ of collisional energy attributed to spin-orbit transitions leading to spin flip (Stowe et al. 1990).

\section{Appendix B: Reaction $\mathrm{S}\left({ }^{3} \mathbf{P}\right)+\mathrm{H}_{2}(v) \rightleftarrows \mathrm{SH}+\mathbf{H}$}

This reaction involves open shell reactants, $\mathrm{S}\left({ }^{3} P\right)$, and products, $\mathrm{SH}\left({ }^{2} \Pi\right)$. Neglecting spin flipping, there are three states that correlate to $\mathrm{S}\left({ }^{3} P\right)$, two of them connect to the $\mathrm{SH}\left({ }^{2} \Pi\right)$. These two electronic states are of ${ }^{3} A^{\prime}$ and ${ }^{3} A^{\prime \prime}$ symmetry, and have been studied in detail by Maiti et al. (2004). Here we use the adiabatic PES calculated by Maiti et al. (2004). Reaction $\mathrm{S}+\mathrm{H}_{2} \rightarrow \mathrm{SH}+\mathrm{H}$ is endothermic by $\simeq 1.02 \mathrm{eV}$ (without zero-point energy corrections), very similar to the endothermicity of reaction $\mathrm{S}^{+}+\mathrm{H}_{2} \rightarrow \mathrm{SH}^{+}+\mathrm{H}$ (Zanchet et al. 2013a, 2019). The main difference is the presence of a barrier, of $\simeq 78 \mathrm{meV}(\simeq 905 \mathrm{~K})$ with respect to the $\mathrm{SH}+\mathrm{H}$ asymptote.

We performed quantum wave packet calculations for the reactions $\mathrm{S}+\mathrm{H}_{2}(v=2,3, j=0)$ and $\mathrm{SH}(v=0, j=0)+\mathrm{H}$. We used MADWAVE3 (Gómez-Carrasco \& Roncero 2006; Zanchet et al. 2009) to calculate the reaction probabilities for the initial vibrational state of the diatomic reactant (in the ground state rotational state, $j=0)$. We employed the usual partial wave expansion to calculate the reaction cross section. We calculated only few total angular momenta of the triatomic system, $J=0,10$ and 20 . The other $J$ needed in the partial wave expansion were obtained using the $J$-shifting-interpolation method (see Zanchet et al. 2013a). The initial-state-specific rate constants are obtained by numerical integration of the cross section using a Boltzmann distribution (Zanchet et al. 2013a). The resulting reaction rate constants are shown in Figs. B.1 and B.2. The numerical values of the rate constants are fitted to the usual analytical Arrhenius-like expresion (shown as dotted curves). We note that the shoulder in the rate constants of reaction $\mathrm{SH}(v=0)+\mathrm{H}$ requires two functions in the temperature range of 200-800 K. Rate coefficients are tabulated in Table 1.

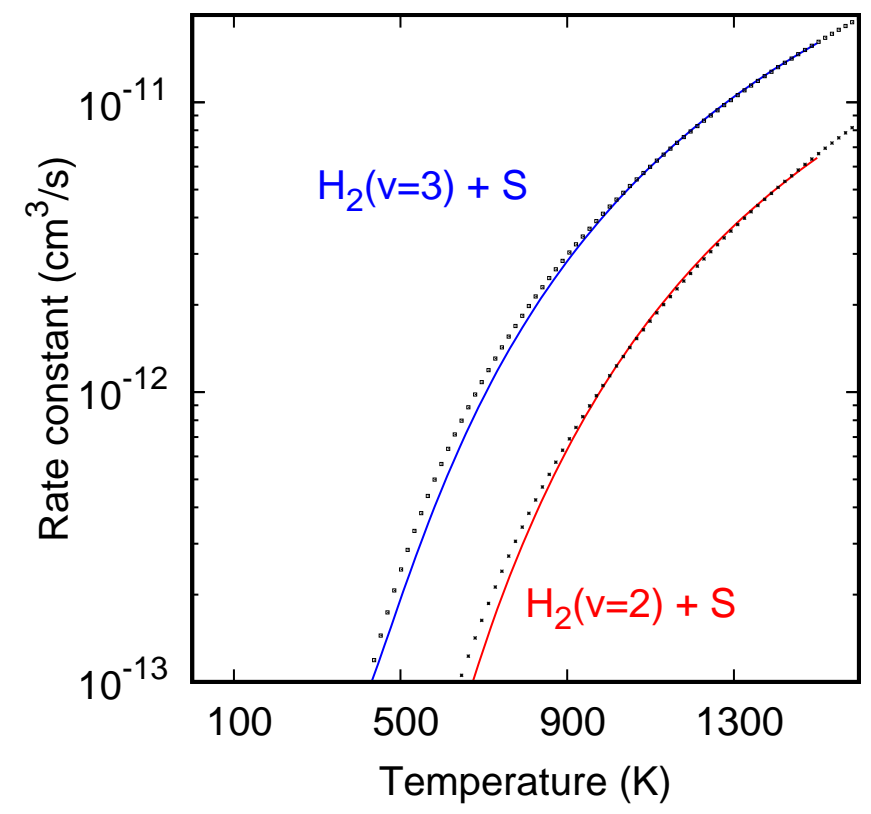

Fig. B.1. Calculated rate constants as a function of temperature for reaction $\mathrm{S}\left({ }^{3} P\right)+\mathrm{H}_{2}(v) \rightarrow \mathrm{SH}+\mathrm{H}$. Dotted curves are fits of the form $k(T)=\alpha(T / 300)^{\beta} \exp (-\gamma / T)$. Rate coefficients are listed in Table 1.

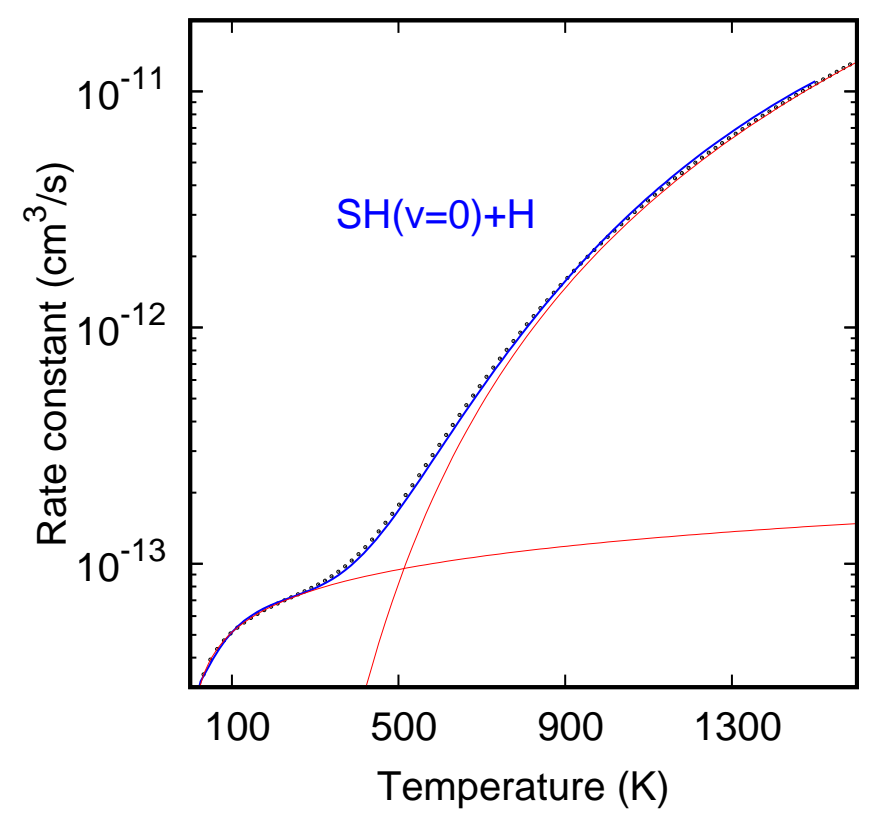

Fig. B.2. Calculated rate constants as a function of temperature for reaction $\mathrm{SH}(v=0)+\mathrm{H} \rightarrow \mathrm{S}+\mathrm{H}_{2}$. The best fit to the calculated rate requires two Arrhenius-like expressions (one for low temperatures and one for high temperatures). Rate coefficients of these fits are listed in Table 1. 


\section{Appendix $\mathrm{C}: \mathrm{SH}$ and $\mathrm{H}_{2} \mathrm{~S}$ photoionization and photodissociation cross sections}

Figure C. 1 shows the experimental $\mathrm{SH}$ and $\mathrm{H}_{2} \mathrm{~S}$ photoionization and photodissociation cross sections $\left(\mathrm{cm}^{-2}\right)$ used in our PDR models. We integrate these cross sections over the specific FUV radiation field at each $A_{V}$ depth of the PDR to obtain the specific photoionization and photodissociation rates $\left(\mathrm{s}^{-1}\right)$.
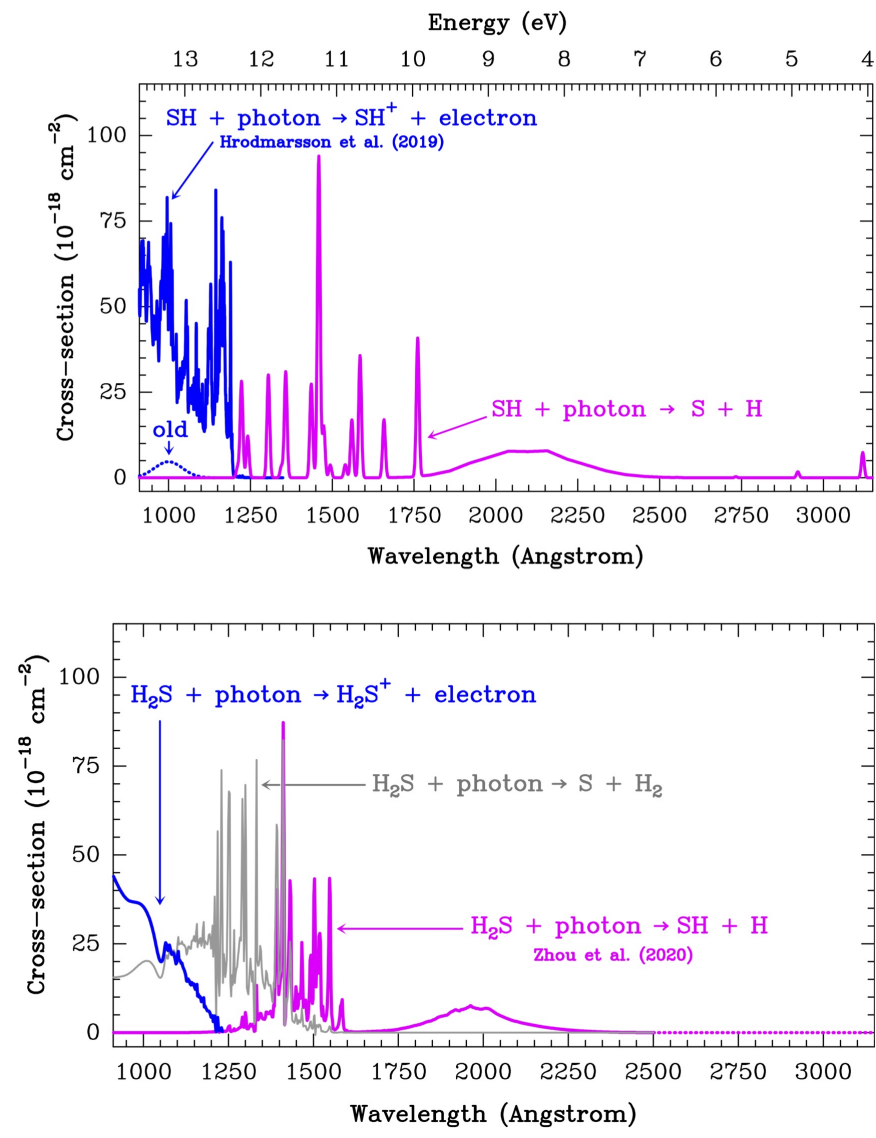

Fig. C.1. Photoionization and photodissociation cross sections. Top panel: $\sigma_{\text {ion }}(\mathrm{SH})$ (blue curve from laboratory experiments by Hrodmarsson et al. 2019). The pink curve is $\sigma_{\text {diss }}(\mathrm{SH})$ (Heays et al. 2017, and references therein). Bottom panel: $\sigma_{\text {ion }}\left(\mathrm{H}_{2} \mathrm{~S}\right)$ (blue curve) and $\sigma_{\text {diss }}\left(\mathrm{H}_{2} \mathrm{~S}\right)$ (gray and pink curves; from Zhou et al. 2020).

\section{Appendix D: $\mathrm{H}_{\mathbf{2}} \mathrm{S}$ ortho-to-para ratio and $\boldsymbol{T}_{\text {spin }}$}

The OTP ratio is sometimes related to a nuclear-spin-temperature ( $T_{\text {spin }}$, e.g., Mumma et al. 1987) defined, for $\mathrm{H}_{2} \mathrm{O}$ or $\mathrm{H}_{2} \mathrm{~S}$, as:

$\mathrm{OTP}=\frac{3 \sum(2 J+1) \exp \left(-E_{o}(J) / T_{\text {spin }}\right)}{\sum(2 J+1) \exp \left(-E_{p}(J) / T_{\text {spin }}\right)}$.

Here, $E_{o}(J)$ and $E_{p}(J)$ are the energies (in Kelvin) of $o-\mathrm{H}_{2} \mathrm{~S}$ and $p-\mathrm{H}_{2} \mathrm{~S}$ rotational levels (with the two ground rotational states separated by $\Delta E=19.8 \mathrm{~K}$ ). Figure D. 1 shows the OTP ratio of the two $\mathrm{H}_{2} \mathrm{~S}$ nuclear spin isomers as a function of $T_{\text {spin. }}$. The OTP ratio we infer toward the DF position of the Bar, $2.9 \pm 0.3$, is consistent with the statistical ratio of $3 / 1$, and implies $T_{\text {spin }} \geq 30 \pm 10 \mathrm{~K}$.

\section{Appendix E: Line parameters of IRAM 30m, ALMA, and SOFIA observations}

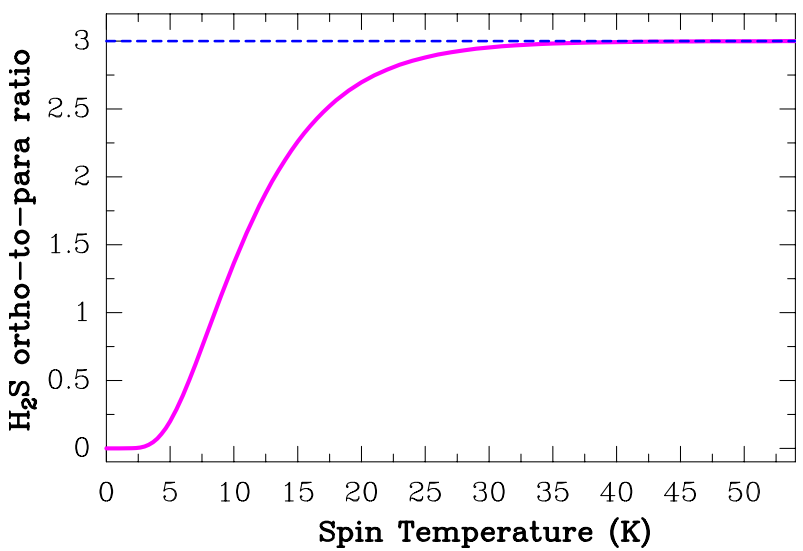

Fig. D.1. OTP ratio of $\mathrm{H}_{2} \mathrm{~S}$ as a function of spin temperature (eq. D.1). 
Table E.1. Parameters of $\mathrm{H}_{2} \mathrm{~S}$ and $\mathrm{H}_{2}^{34} \mathrm{~S}$ lines detected with the IRAM $30 \mathrm{~m}$ telescope toward three positions of the Orion Bar.

\begin{tabular}{lccccccccccc}
\hline \hline Position & \multirow{2}{*}{ Species } & $\begin{array}{c}\text { Transition } \\
\text { Frequency }\end{array}$ & $\begin{array}{c}E_{\mathrm{u}} / \mathrm{k} \\
{[\mathrm{GHz}]}\end{array}$ & $\begin{array}{c}A_{\mathrm{ul}} \\
{[\mathrm{K}]}\end{array}$ & $\begin{array}{c}S_{\mathrm{ul}} \\
{\left[\mathrm{s}^{-1}\right]}\end{array}$ & $\begin{array}{c}g_{\mathrm{u}} \\
J_{K_{\mathrm{a}}, K_{\mathrm{c}}}\end{array}$ & $\begin{array}{c}\int T_{\mathrm{mb}} \mathrm{dv} \\
{\left[\mathrm{K} \mathrm{km} \mathrm{s}^{-1}\right]}\end{array}$ & $\begin{array}{c}\mathrm{v}_{\mathrm{LSR}} \\
{\left[\mathrm{km} \mathrm{s}^{-1}\right]}\end{array}$ & $\begin{array}{c}\Delta \mathrm{v} \\
{\left[\mathrm{km} \mathrm{s}^{-1}\right]}\end{array}$ & $\begin{array}{c}T_{\mathrm{mb}} \\
{[\mathrm{K}]}\end{array}$ \\
\hline \multirow{2}{*}{$+10,-10)$} & $o-\mathrm{H}_{2} \mathrm{~S}$ & $1_{1,0}-1_{0,1}$ & 168.763 & 8.1 & $2.68 \times 10^{-5}$ & 1.5 & 3 & $18.32(0.01)$ & $10.5(0.1)$ & $2.5(0.1)$ & 7.03 \\
& $o-\mathrm{H}_{2}^{34} \mathrm{~S}$ & $1_{1,0}-1_{0,1}$ & 167.911 & 8.1 & $2.62 \times 10^{-5}$ & 1.5 & 3 & $1.22(0.01)$ & $10.5(0.1)$ & $2.0(0.1)$ & 0.57 \\
& $p-\mathrm{H}_{2} \mathrm{~S}$ & $22,0-2_{1,1}$ & 216.710 & 84.0 & $4.87 \times 10^{-5}$ & 2.2 & 5 & $0.35(0.01)$ & $10.4(0.1)$ & $2.1(0.1)$ & 0.16 \\
\hline \multirow{2}{*}{$+30,-30)$} & $o-\mathrm{H}_{2} \mathrm{~S}$ & $1_{1,0}-1_{0,1}$ & 168.763 & 8.1 & $2.68 \times 10^{-5}$ & 1.5 & 3 & $17.16(0.02)$ & $10.3(0.1)$ & $2.4(0.1)$ & 6.85 \\
& $o-\mathrm{H}_{2}^{34} \mathrm{~S}$ & $1_{1,0}-1_{0,1}$ & 167.911 & 8.1 & $2.62 \times 10^{-5}$ & 1.5 & 3 & $1.28(0.01)$ & $10.4(0.1)$ & $1.9(0.1)$ & 0.63 \\
\hline \multirow{2}{*}{$+35,-55)$} & $o-\mathrm{H}_{2} \mathrm{~S}$ & $1_{1,0}-1_{0,1}$ & 168.763 & 8.1 & $2.68 \times 10^{-5}$ & 1.5 & 3 & $3.57(0.02)$ & $9.6(0.1)$ & $3.1(0.1)$ & 1.08 \\
& $o-\mathrm{H}_{2}^{34} \mathrm{~S}$ & $1_{1,0}-1_{0,1}$ & 167.911 & 8.1 & $2.62 \times 10^{-5}$ & 1.5 & 3 & $0.18(0.02)$ & $9.8(0.1)$ & $2.7(0.3)$ & 0.06 \\
\hline
\end{tabular}

Notes. Parentheses indicate the uncertainty obtained by the Gaussian fitting programme.

Table E.2. Parameters of $\mathrm{SH}^{+}$targeted with ALMA toward the DF position.

\begin{tabular}{|c|c|c|c|c|c|c|c|c|c|}
\hline Position & Species & Transition & $\begin{array}{c}\text { Frequency } \\
{[\mathrm{GHz}]}\end{array}$ & $\begin{array}{l}E_{\mathrm{u}} / \mathrm{k} \\
{[\mathrm{K}]}\end{array}$ & $\begin{array}{c}A_{\mathrm{ul}} \\
{\left[\mathrm{s}^{-1}\right]}\end{array}$ & $\underset{\left[\mathrm{K} \mathrm{km} \mathrm{s}^{-1}\right]}{\int T_{\mathrm{mb}} \mathrm{dv}}$ & $\begin{array}{c}\mathrm{v}_{\mathrm{LSR}} \\
{\left[\mathrm{km} \mathrm{s}^{-1}\right]}\end{array}$ & $\begin{array}{c}\Delta \mathrm{v} \\
{\left[\mathrm{km} \mathrm{s}^{-1}\right]}\end{array}$ & $\begin{array}{r}T_{\mathrm{mb}} \\
{[\mathrm{K}]}\end{array}$ \\
\hline \multirow[t]{2}{*}{$(+10,-10)$} & $\mathrm{SH}^{+}$ & $N_{J}=1_{0^{-}} 0_{1} \quad F=1 / 2-1 / 2$ & 345.858 & 16.6 & $1.14 \times 10^{-4}$ & $0.36^{a}(0.03)$ & $10.7(0.2)$ & $2.7(0.3)$ & 0.12 \\
\hline & $\mathrm{SH}^{+}$ & $N_{J}=1_{0}-0_{1} F=1 / 2-3 / 2^{b}$ & 345.944 & 16.6 & $2.28 \times 10^{-4}$ & $0.70^{a}(0.03)$ & $10.4(0.1)$ & $2.5(0.1)$ & 0.26 \\
\hline
\end{tabular}

Notes. ${ }^{a}$ Integrated over a $5^{\prime \prime}$ aperture to increase the $\mathrm{S} / \mathrm{N}$ of the line profiles. ${ }^{b}$ Line integrated intensity map shown in Fig. 3.

Table E.3. Parameters of SH lines (neglecting HFS) targeted with SOFIA toward the DF position.

\begin{tabular}{|c|c|c|c|c|c|c|c|c|c|}
\hline Position & Species & Transition & $\begin{array}{c}\text { Frequency } \\
{[\mathrm{GHz}]}\end{array}$ & $\begin{array}{c}E_{\mathrm{u}} / \mathrm{k} \\
{[\mathrm{K}]}\end{array}$ & $\begin{array}{c}A_{\mathrm{ul}} \\
{\left[\mathrm{s}^{-1}\right]}\end{array}$ & $\underset{\left[\mathrm{K} \mathrm{km} \mathrm{s}^{-1}\right]}{\int_{\mathrm{mb}} \mathrm{dv}}$ & $\begin{array}{c}\mathrm{v}_{\mathrm{LSR}} \\
{\left[\mathrm{km} \mathrm{s}^{-1}\right]}\end{array}$ & $\begin{array}{c}\Delta \mathrm{v} \\
{\left[\mathrm{km} \mathrm{s}^{-1}\right]}\end{array}$ & $\begin{array}{l}T_{\mathrm{mb}} \\
{[\mathrm{K}]}\end{array}$ \\
\hline \multirow[t]{2}{*}{$(+10,-10)$} & $\mathrm{SH}$ & ${ }^{2} \Pi_{3 / 2} J=5 / 2^{+}-3 / 2^{-}$ & 1382.911 & 66.4 & $4.72 \times 10^{-3}$ & $<1.11^{a}(0.20)$ & $12.1^{a}(0.8)$ & $7.9^{a}(1.3)$ & 0.16 \\
\hline & $\mathrm{SH}$ & ${ }^{2} \Pi_{3 / 2} J=5 / 2^{-}-3 / 2^{+}$ & 1383.242 & 66.4 & $4.72 \times 10^{-3}$ & $<0.34(0.12)$ & $11.7(0.5)$ & $2.3(0.8)$ & 0.14 \\
\hline
\end{tabular}

Notes. ${ }^{a}$ Uncertain fit. 